\title{
Raman scattering in symbiotic stars. I. Spectropolarimetric observations
}

\author{
T.J. Harries ${ }^{1}$ and I.D. Howarth ${ }^{2}$ \\ 1 School of Physics and Astronomy, University of St. Andrews, North Haugh, St. Andrews, Fife KY16 9SS, Scotland \\ 2 Dept. of Physics and Astronomy, University College London, Gower Street, London WC1E 6BT, UK
}

Received October 16; accepted December 9, 1995

\begin{abstract}
We present high signal-to-noise, high-dispersion linear spectropolarimetry of the Raman-scattered $\lambda \lambda 6825,7082 \AA$ emission lines in 27 southern-hemisphere symbiotic systems. We give continuum polarization measurements for all the targets (together with estimates of the foreground interstellar polarization), and new estimates of the red-giant spectral types in 23 systems. We confirm continuum-polarization variability in five out of eight systems with previous high-quality photopolarimetry. The Raman lines are fully resolved; although they display a wide variety of morphologies, both the intensity and polarization profiles appear basically to be triple-peaked. Spectra of five systems observed in both 1992 and 1994 show stable Raman-line intensity profiles, but with significant variations in the polarization structure.
\end{abstract}

Key words: stars — stars: binaries: symbiotic — stars: late-type — stars: mass-loss — polarization

\section{Introduction}

It is generally accepted that symbiotic stars are binary systems consisting of a hot star which ionizes material shed by a cool, giant companion, resulting in a characteristic combination spectrum of sharp, nebular emission lines and stellar molecular absorption bands (e.g., Kenyon 1986). The binary periods are typically on the order of years and upwards, and hence accurate masses have been established for a only a handful of systems. Furthermore, the mechanisms that drive the mass outflows from the red-giant stars are poorly understood (both in symbiotic systems and in isolated objects). It is therefore highly desirable to obtain observational constraints on both the binary geometry and wind structure of these systems.

In a seminal paper, Schmid (1989) attributed two previously unidentified nebular lines at $\sim \lambda \lambda 6825,7080 \AA$ to the result of Raman scattering of resonance-line $\mathrm{O}$ VI photons $(\lambda \lambda 1032,1038 \AA)$, originating in the ionized nebula, off of neutral hydrogen associated with the cool giant. Raman scattering is the inelastic analogue of Rayleigh scattering; the frequency of the scattered photon is different to that of the incident photon, and the scatterer is left in an altered quantum-mechanical state. In this case, the $\mathrm{O}$ VI photons scatter in the far-red wing of Ly $\beta$ and the scattering $\mathrm{H}^{0}$ atom is left in the $n=2$ state. By conservation of energy the frequency of the Raman photon, $\nu_{\mathrm{r}}$,

Send offprint requests to: T.J. Harries (tjh@st-and.ac.uk) is given by

$$
\nu_{\mathrm{r}}=\nu_{\mathrm{p}}-\nu_{\mathrm{Ly} \alpha}
$$

where $\nu_{\mathrm{Ly} \alpha}$ is the frequency of the Ly $\alpha$ transition and $\nu_{\mathrm{p}}$ is the frequency of the parent photon (in this case $\lambda 1032$ or $\lambda 1038$ ), giving true line wavelengths of $\lambda \lambda 6827.33$, $7084.39 \AA$ in vacuum and $\lambda \lambda 6825.45,7082.44 \AA$ in air.

Raman scattering occurs according to the Rayleighscattering phase matrix, and since the scattering geometry in the symbiotic system is markedly asymmetric (as photons from the nebula are scattered in the locale of the red giant), the emission lines are expected to show significant linear polarization. Observations by Schmid \& Schild (1990) confirmed this hypothesis, with linear polarizations of $5.2 \%$ and $9.4 \%$ reported for the $\lambda \lambda 6825,7082 \AA$ lines in the spectrum of the symbiotic Mira He2-38. Further observations of 15 symbiotic systems (Schmid \& Schild 1994, hereinafter SS94) revealed that a high proportion of symbiotic systems display enhanced polarization in the Raman lines, and that the polarization structure through the lines can be complex, with rotations and discontinuities in the position angle (PA) and with multiple polarization peaks.

The Raman-scattered lines offer a powerful new diagnostic tool for investigating the geometry of the scattering regime. Recent observations (SS94; this paper) show that the scattering must occur in an extended, expanding wind, so that spectropolarimetry of the lines affords an opportunity to study the mass-loss characteristics of 
the cool components as well as the binary parameters of the systems. The SS94 survey was hampered by relatively low $\mathrm{S} / \mathrm{N}$ and poor resolution (often only a few resolution elements per line). Here we present a homogeneous, high-dispersion, high-S/N spectropolarimetric survey of the Raman-scattered emission lines of 27 southernhemisphere symbiotic stars. This sample represents rather more than half of the total number of Galactic symbiotics listed by Kenyon (1986) as showing the $\lambda 6825$ line.

\section{Observations}

The majority of the observations were obtained using the 3.9-m Anglo-Australian telescope on the nights of 1994 May 6-10, with additional exploratory data (discussed in Sect. 5.6) obtained in 1992 March. The RGO cassegrain spectrograph was used in conjunction with the polarimetry module, which consists of a superachromatic halfwave plate and a calcite analyzer. The 25 -cm camera and $1200 \mathrm{~V}$ grating were employed, and a $1024 \times 1024$ pixel Tektronics CCD used as the detector. The mean reciprocal dispersion was $0.79 \AA$ pixel $^{-1}$ while the resolution, obtained from the mean FWHM of the arc calibration lines, was found to be $1.3 \AA\left(\approx 55 \mathrm{~km} \mathrm{~s}^{-1}\right)$. The spectra cover the range $\sim 6600-7400 \AA$ (with a blocking filter used to eliminate second-order blue signal); $\mathrm{H} \alpha$ was deliberately excluded to avoid saturating the detector.

The targets were chosen from Allen's (1984) catalogue. Aside from positional constraints, the selected targets had to satisfy just two criteria: i) evidence for Ramanscattered lines in the low-dispersion spectra presented in the Allen catalogue or listed as having $\lambda 6825$ emission by Kenyon (1986); and ii) bright enough to allow a high $\mathrm{S} / \mathrm{N}$ observation in a reasonable time (typically eight 300 second exposures). We also included RX Pup in our programme, even though neither Allen nor Kenyon indicate the presence of the $\lambda 6825 \AA$ line, because it is a relatively bright D-type system for which Schmid \& Schild (1990) have reported $\lambda 6825 \AA$ emission.

With these constraints, we obtained data for 27 symbiotic stars in the 1994 run; a summary list of objects is given in Table 1. The target list contains systems with a wide range of spectral types for the cool-giant components, including the carbon Mira AS 210. Most of the targets are S-type (i.e., have stellar IR flux distributions), but nine are D-type systems (i.e., have IR flux distributions which show strong dust signatures), all of which (except AS 276) are listed by Whitelock (1987) as having Mira-type cool components.

\section{Data reduction}

Each polarization measurement consisted of a set of four exposures corresponding to the four waveplate positions $\left(0^{\circ}, 22.5,45^{\circ}\right.$, and 67.5$)$. The dekker used has a pair of apertures, 'A' and 'B', for the sky and target. A typical observation consisted of two sets of four images: four exposures, corresponding to four waveplate positions, with the target in the A aperture, then four with it in the B aperture. Several standard stars were observed for calibration purposes and additional calibration spectra were obtained through an HN22 filter, which is nearly $100 \%$ polarizing at the wavelengths of interest. For most symbiotic targets we obtained $10^{4}-10^{5}$ or more continuum counts per channel.

Preliminary data reduction was performed by using the FIGARO package (Meyerdicks 1992). The mean bias, found from the overscanned bias strip, was subtracted from all the images and the frames were flatfielded with a balanced flatfield. The locations of the ordinary (o) and extraordinary (e) spectra on each image were found by collapsing the frame in the dispersion direction. The target and sky spectra were obtained by collapsing the image in the cross-dispersion direction and the sky spectra were subtracted from the stellar spectra.

Cross-talk between the o and e channels will reduce the contrast between the respective measured intensities, and hence the calculated polarization. A potential source of such cross-talk is cross-dispersion scattering, and so a first-order correction was made to minimize the effects of such scattering. If $X_{\mathrm{o}}, X_{\mathrm{e}}$ are the locations of the o and e spectra on the detector in the cross-dispersed direction (in principle, a function of $\lambda$, but in practice constant to a fraction of a pixel), then for each o spectrum a second 'spectrum' was extracted at $X_{\mathrm{o}}-\left(X_{\mathrm{e}}-X_{\mathrm{o}}\right)$. Assuming that the spatial scattering profile is symmetric, this spectrum should have the same intensity as the light scattered into the e spectrum from the o channel, and may be used to correct for any such scattering. The o signal may be similarly treated. (It was not possible to use this method for channels falling close to the edge of the detector frame, and in those cases a fraction of the neighbouring spectrum corresponding to the ratio between a scattered-flux spectrum and intensity spectrum extracted elsewhere on the image was employed.) In practice, these precautions resulted in only very slight changes to the polarimetry data.

The o and e spectra were separately wavelength calibrated by using low-order polynomial fits to the appropriate arc spectra, since the polarization module introduces a small wavelength shift between the o and e rays. (If the same calibration is used for both the o and e spectra then spurious spikes may appear in the polarization data, resulting from mismatched sharp spectral intensity gradients such as result from absorption edges or narrow spectral lines.) The o and e spectra were then resampled onto a common wavelength grid for subsequent processing.

The ratio of the o and e spectra at each wavelength sample may be used to obtain one Stokes parameter. However the intensities of the two spectra are dependent on the system gain factors such as grating efficiencies, mirror reflection coefficients, and pixel-to-pixel detector sensitivity. In order to compensate for the system gain a second 
Table 1. The target list; designations follow Allen (1984; Hen 1242 = KX TrA)

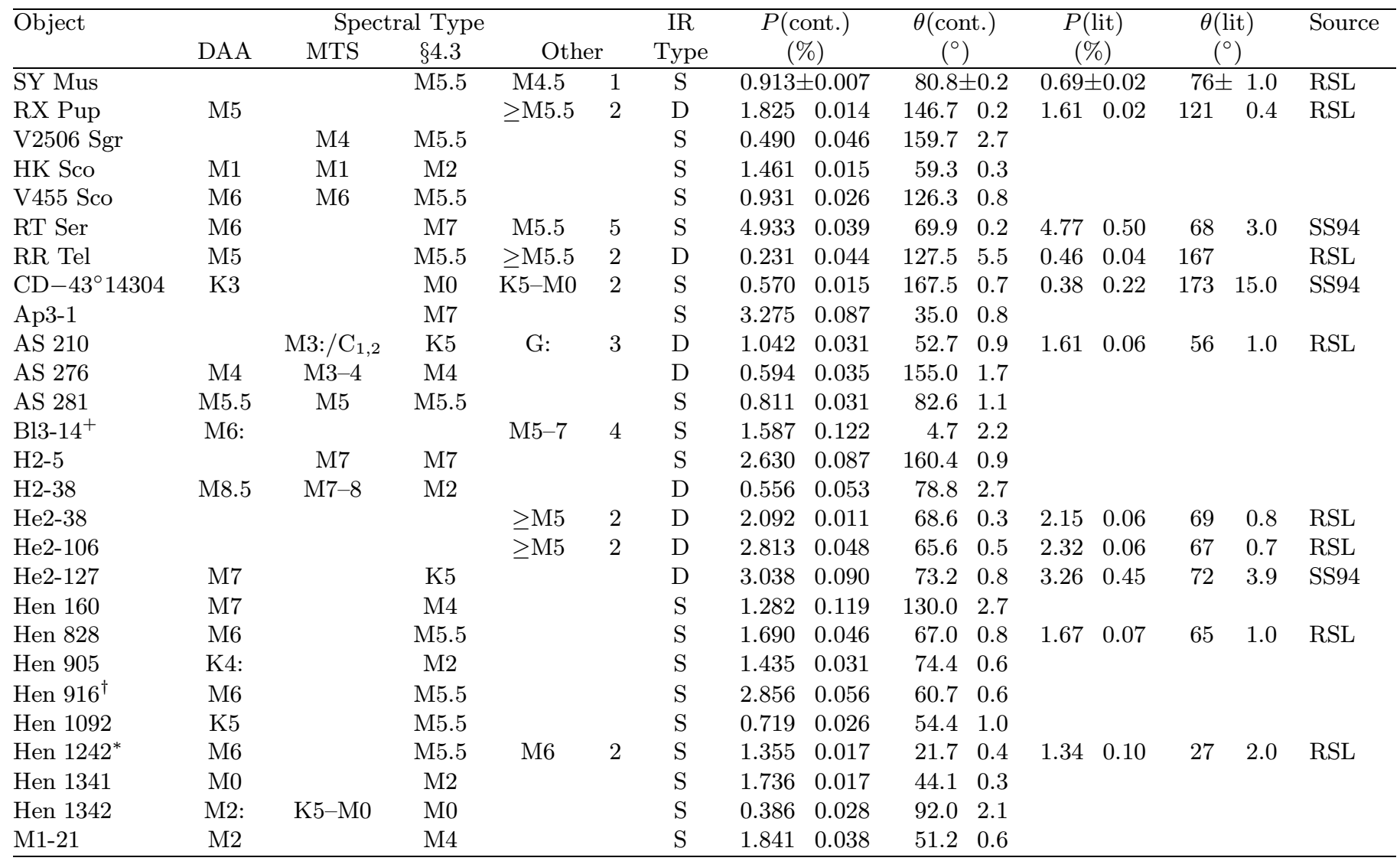

Finding-chart errors in Allen (1984):

${ }^{*} 3 \mathrm{~mm}\left(\approx 25^{\prime \prime}\right) \mathrm{W}$ of marked star (tabulated co-ordinates are correct)

${ }^{\dagger} 2 \mathrm{~mm}\left(\approx 20^{\prime \prime}\right)$ E of marked star.

${ }^{+}$Correctly identified in the finder chart, but our telescope co-ordinates (17h 52m 06.4s, -29 $45^{\prime} 49^{\prime \prime}$, B1950) disagree with the tabulated position ( $\left.17 \mathrm{~h} 49 \mathrm{~m} 14.1 \mathrm{~s},-29^{\circ} 45^{\prime} 19^{\prime \prime}\right)$.

Spectral types are taken from Allen (1980b; DAA), Medina Tanco \& Steiner (1995; MTS), and from (1) Schmutz et al. (1994), (2) Schulte-Ladbeck (1988), (3) Allen (1981), (4) Gutiérrez-Moreno \& Moreno (1995), or (5) Kenyon \& Fernandez-Castro (1987), and are listed together with our estimates (from Sect. 4.3). The 'D' and 'S' ('dust' and 'star') IR-type assignments are from Allen (1984).

Continuum polarization measurements from the AAT observations are listed, together with literature values $(\mathrm{RSL}=R$-band photopolarimetry from Schulte-Ladbeck et al. 1990, except RR Tel ( $I$-band measurement from Schulte-Ladbeck \& Magalhães 1987); SS94 = spectropolarimetry from Schmid \& Schild 1994).

exposure was made with the waveplate rotated by $45^{\circ}$, which inverts the sign of the Stokes parameter. All the polarization-dependent effects are thus inverted, while the system gain remains constant. The recorded intensities $i$ can be written

$$
\begin{aligned}
i_{0, \|} & =\frac{1}{2}(I+Q) G_{\|} F_{0} \\
i_{0, \perp} & =\frac{1}{2}(I-Q) G_{\perp} F_{0} \\
i_{45, \|} & =\frac{1}{2}(I+Q) G_{\|} F_{45}
\end{aligned}
$$

$$
i_{45, \perp}=\frac{1}{2}(I-Q) G_{\perp} F_{45}
$$

where $G_{\|}$and $G_{\perp}$ are the system gains of the ordinary and extraordinary rays respectively, $F_{0}$ and $F_{45}$ are the timedependent factors corresponding to image-wander and seeing fluctuations of the images obtained at the $0^{\circ}$ and $45^{\circ}$ positions of the half-wave plate, $I$ is the total polarized intensity, and $Q$ is the Stokes parameter. Dividing the o and e spectra in each image cancels the $F$ factors, removing the time-dependent gains. Division of the resulting ratios removes the polarization-dependent gain factors $G$. The use of flatfields is therefore, in principle, superfluous; 
flatfielding is, however, useful 'insurance' against small spatial mismatches in the sets of spectra.

The normalized Stokes parameter $\bar{q}$ is obtained from

$$
\bar{q}=Q / I=\frac{R-1}{R+1}
$$

where

$$
R^{2}=\frac{i_{0, \|} / i_{0, \perp}}{i_{45, \|} / i_{45, \perp}}
$$

The $\bar{u}$ Stokes parameter may be obtained similarly, from measurements obtained at the $22.5^{\circ}$ and $67.5^{\circ}$ positions of the half-wave plate. The normalized Stokes parameters are related to the fractional polarization $P$ and the position angle $\theta$ (measured east from north in the range $0-180^{\circ}$ ) by

$$
\begin{aligned}
P & =\sqrt{\bar{q}^{2}+\bar{u}^{2}}, \\
2 \theta & =\tan ^{-1}(\bar{u} / \bar{q}) .
\end{aligned}
$$

Statistical methods for polarimetric analysis are discussed fully by Clarke \& Stewart (1986), who show that, for situations of low signal-to-noise, the effects of kurtosis in the probability distribution of the errors on $Q$ and $U$ must be taken into account. However, in situations of high $\mathrm{S} / \mathrm{N}$, as in the observations presented in this study, the following treatment is acceptable. If the throughputs for the $0^{\circ}$ and $45^{\circ}$ observations are the same and the fractional polarization of the object is small then

$$
i_{0, \|} \approx i_{0, \perp} \approx i_{45, \|} \approx i_{45, \perp}
$$

and

$$
i_{0, \|}+i_{0, \perp}+i_{45, \|}+i_{45, \perp}=I=4 i
$$

Assuming that photon statistics dominate, we have

$$
\sigma_{i} \approx \sqrt{i}
$$

and so

$$
\frac{\sigma_{R^{2}}}{R^{2}} \approx \frac{2}{\sqrt{i}}
$$

and

$$
\frac{\sigma_{R}}{R} \approx \frac{1}{\sqrt{i}}=\frac{2}{\sqrt{I}}
$$

Since $R \approx 1$ for small fractional polarization

$$
\sigma_{R} \approx \frac{2}{\sqrt{I}},
$$

and, since $R+1 \gg R-1$, we obtain

$$
\frac{\sigma_{\bar{q}}}{\bar{q}} \approx \frac{2}{\sqrt{I}(R-1)},
$$

finally giving

$$
\sigma_{\bar{q}} \approx \frac{2}{\sqrt{I}(R-1)} \frac{R-1}{R+1} \approx \frac{1}{\sqrt{I}}
$$

and

Similarly,

$$
\sigma_{Q} \approx \sqrt{I}
$$

$$
\sigma_{U} \approx \sqrt{I} .
$$

Equations (18), (19) are the basis of the internal errors used in this paper.

The PA zero-point of the observations was calibrated by using polarized standard-star spectra, whilst the PA wavelength dependence (introduced by slight chromaticism of the half-wave plate) was removed by polynomial fits to the HN22-filter observations. The instrumental polarization, obtained from observations of zero polarized standard stars, was found to be less than $0.1 \%$, and was not corrected for. Systematic errors in the polarization errors resulting from incorrect sky subtraction, inadequate scattered-light compensation, and other sources are also expected not to exceed $\sim 0.1 \%$. The polarization spectra of all the symbiotic stars observed are presented in Fig. 9.

\section{Continuum and interstellar polarizations}

\subsection{The continuum polarizations}

The mean continuum polarization was measured for each star in continuum bins of $\lambda \lambda 6700-6750,6900-6950$, $7150-7200$, and $7300-7350 \AA$, selected to avoid the strongest emission lines in the spectral range. The data were summed to give the mean intensity-weighted continuum polarizations,

$$
I_{\mathrm{tot}}=\sum_{\lambda} I_{\lambda} \quad Q_{\mathrm{tot}}=\sum_{\lambda} Q_{\lambda} \quad U_{\mathrm{tot}}=\sum_{\lambda} U_{\lambda} .
$$

These continuum polarization data are listed in Table 1. Also listed are red-region photopolarimetric measures from Schulte-Ladbeck et al. (1990) or SchulteLadbeck \& Magalhães (1987), and the SS94 spectropolarimetric values. Where data are available from both sources, the photopolarimetric measurements are listed preferentially, since the SS94 data have relatively large uncertainties.

There is a formally significant $(>3 \sigma)$ difference between our measurements and published photopolarimetric values of the magnitude of the polarization vector in 5 stars (out of 8): SY Mus, RX Pup, RR Tel, He2-106, and AS 210. (The continuum polarizations presented here all agree with those given by SS94 to within the latter's large errors.) Temporal variability is evidence of intrinsic continuum polarization, and in fact four of these stars are described as intrinsically polarized by Schulte-Ladbeck et al. (1990).

\subsection{The interstellar polarization}

In order to examine the intrinsic line-polarization morphology it is necessary to account for the interstellar polarization (ISP). The only suitable method for estimating 
the ISP in the present case is the field-star method, in which polarization measurements of stars surrounding the target are used to estimate the ISP vector. This method assumes that the structure of the ISP does not vary along similar sight-lines, an assumption which is clearly in error at some level but which is unavoidable. It also assumes that the field stars are intrinsically unpolarized another questionable assumption, given that many of the field stars with catalogued broad-band polarization measurements are B-giant stars with potentially unstable atmospheres. Furthermore, since the magnitude of the ISP roughly scales with the reddening, it is necessary to find field-stars that are located at approximately the same distance as the target.

The polarization catalogues of Mathewson et al. (1978) and Klare \& Neckel (1977) were searched for stars within a given angular radius of the target star on the plane of the sky. This radius was chosen in order that sufficient field stars were enclosed to define the ISP vector. The spectral types of the stars within this region were found from the Michigan catalogue of two-dimensional spectral types and photometry was obtained from catalogues of ubvy $\beta$ photometry (Hauck \& Mermilliod 1990) or UBV photometry (Mermilliod 1987).

The absolute magnitudes and reddenings of the field stars were obtained by using two methods. A modified version of the code described by Moon (1985) was used for stars for which full $u b v y \beta$ photometry was available. This code employs empirical calibrations of Strömgren photometry (Crawford 1975, 1978, and 1979) and dereddening expressions (e.g., Crawford 1975) in order to estimate the dereddened colour indices, the absolute magnitude, the temperature, and the radius of the star. The $Q$ method (e.g., Heintze 1973) was employed when only $U B V$ photometry was available, with absolute magnitudes taken from Allen (1973).

Spectroscopic parallaxes were estimated for the S-type symbiotics for which $V$ magnitudes were available from Munari et al. (1992) by using absolute magnitudes from C.W. Allen (1973). The reddening for these stars was obtained iteratively from the $A_{V}-\left(m_{0}-M\right)$ relationship defined by the field stars. For D-type systems we adopted distances from Whitelock (1988). The distances adopted are listed in Table 2 . Only field stars within \pm 2 magnitudes of the target-star distance moduli were selected for most symbiotics. H2-38, He2-127, AS 210, Hen 160, RT Ser, RR Tel, and CD $-43^{\circ} 14304$ had no field stars within this range, and the tolerance was widened to \pm 3 magnitudes for $\mathrm{H} 2-38$ and $\mathrm{RT}$ Ser, \pm 6 magnitudes for RR Tel, Hen 1341, CD-4314304, AS 210 and Hen 160, and \pm 8 magitudes for He2-127. All field stars within the adopted angular radius were used for symbiotics without distance estimates, although it is expected that some of these stars will be much nearer than the target star, and will therefore trace only a small fraction of the ISP. The polarization maps are shown in Fig. 1.

Table 2. Distances to the target stars derived in this study (HH) and by Whitelock (1988, W)

\begin{tabular}{lcllcl}
\hline Object & $\begin{array}{c}\text { Dist. } \\
(\mathrm{kpc})\end{array}$ & Ref. & Object & $\begin{array}{c}\text { Dist. } \\
(\mathrm{kpc})\end{array}$ & Ref. \\
\hline SY Mus & 1.4 & $\mathrm{HH}$ & H2-38 & $8.1^{*}$ & W \\
RX Pup & 1.3 & W & He2-38 & 2.8 & W \\
V2506 Sgr & 1.9 & HH & He2-106 & 2.8 & W \\
HK Sco & 2.9 & HH & He2-127 & $14^{*}$ & W \\
V455 Sco & 2.6 & HH & Hen 160 & 9.5 & HH \\
RT Ser & 10.4 & HH & Hen 828 & 2.2 & HH \\
RR Tel & 2.6 & W & Hen 905 & 1.7 & HH \\
CD-43 ${ }^{\circ} 14304$ & 1.0 & HH & Hen 916 & 1.7 & HH \\
Ap3-1 & - & & Hen 1092 & 3.1 & HH \\
AS 210 & $4.9^{*}$ & W & Hen 1242 & 3.8 & HH \\
AS 276 & 2.3 & HH & Hen 1341 & 2.0 & HH \\
AS 281 & 2.1 & HH & Hen 1342 & 2.7 & HH \\
Bl3-14 & - & & M1-21 & 2.7 & HH \\
H2-5 & - & & & & \\
\hline
\end{tabular}

* Distances described as highly uncertain by Whitelock (1988).

Schulte-Ladbeck et al. (1990) suggest that SY Mus, RX Pup, AS 210, He2-38, and He2-106 are intrinsically polarized, on the basis of polarization variability or of a polarization wavelength dependence inconsistent with a Serkowski law. The polarization maps of RX Pup and He2-38 (Figs. 1(ii) and (xvi)) show that the continuumpolarization vectors are inconsistent with the surrounding ISP, suggesting an intrinsic component. The maps for SY Mus, He2-106, and AS 210 (Figs. 1(i), (xvii), and $(x)$ ) are more ambiguous; the continuum polarization of the targets are of a similar magnitude and direction to the field-star ISPs. We conclude that for these stars the observed continuum polarization is probably dominated by the ISP, and that any intrinsic polarization is small. SS94 concluded that the continuum polarization at $\approx 7000 \AA$ for He2-106 and SY Mus is of interstellar origin - a conclusion which is supported by our observations.

The stars which were not observed by Schulte-Ladbeck et al. (1990) and which have continuum polarization vectors that are obviously inconsistent with the field-star ISP are Hen 160, V455 Sco, Hen 1342, Ap3-1, and RR Tel. This inconsistency is evidence for an intrinsic polarization component (notwithstanding the aforementioned caveats regarding the weaknesses of the field-star method). The ISPs for these stars were estimated from the surrounding field stars, in this case by adopting the mean polarization of those stars.

We found no useful polarimetric observations of the remaining stars in the literature, and, since these have continuum polarization vectors that are consistent with the ISP, we treat the continuum polarization as interstellar 

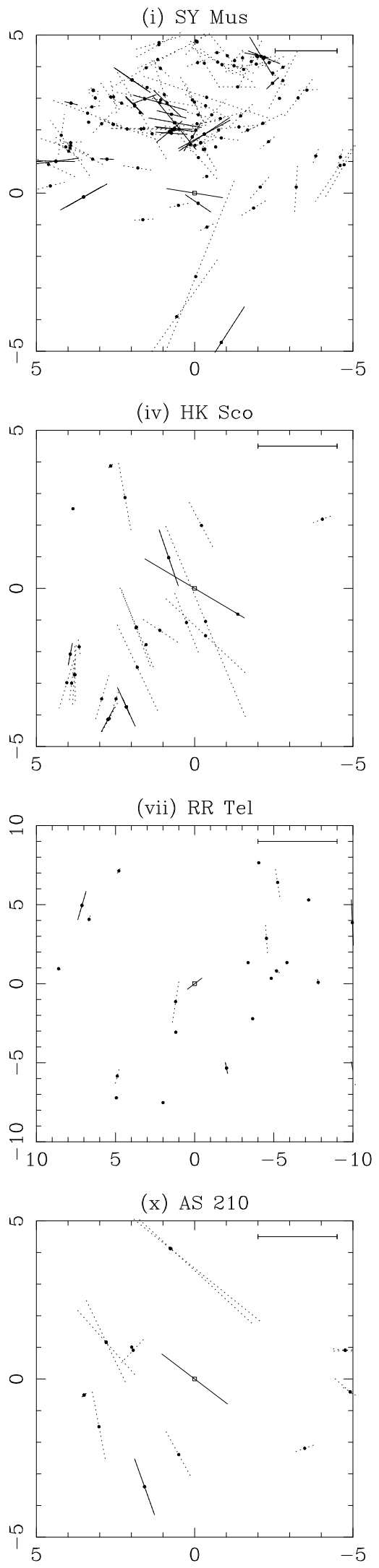

(ii) RX Pup

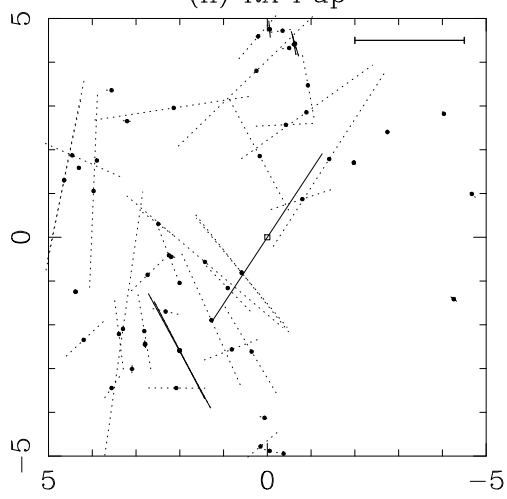

(v) V455 Sco
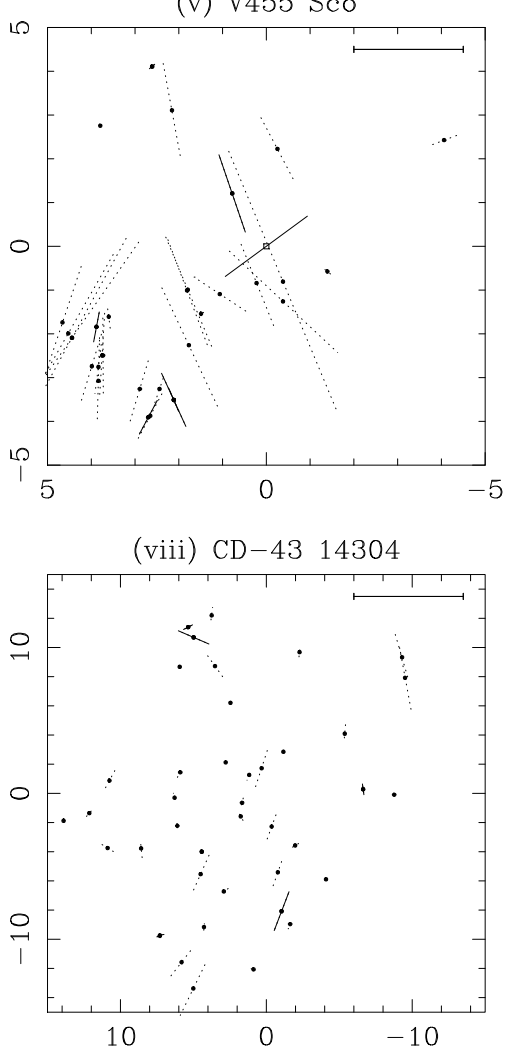

(xi) AS 276

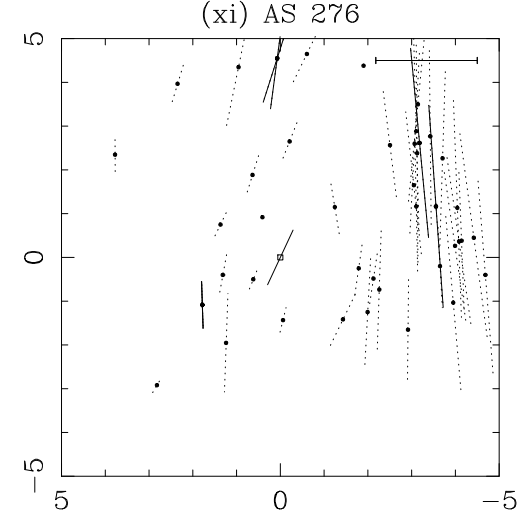

(iii) V2506 Sgr

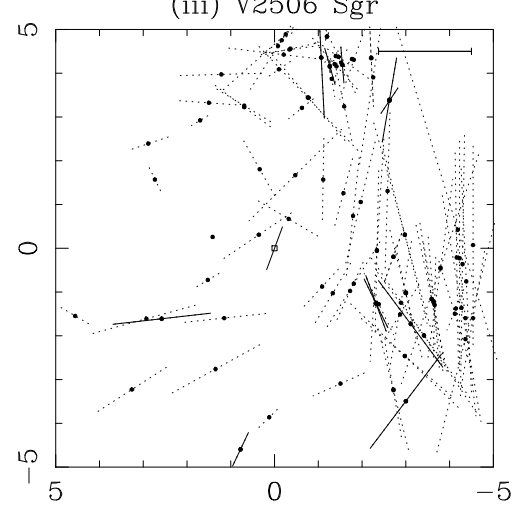

(vi) RT Ser

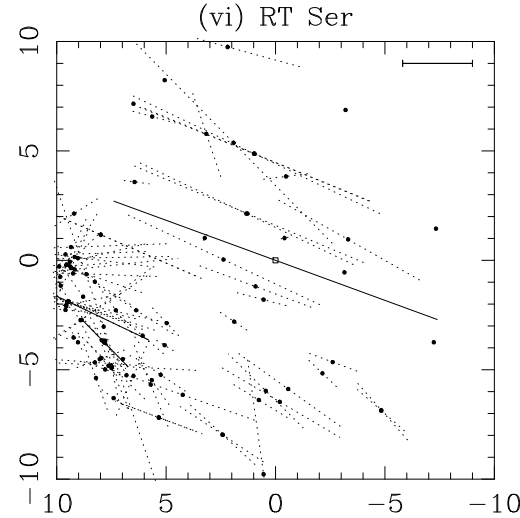

(ix) Ap3-1

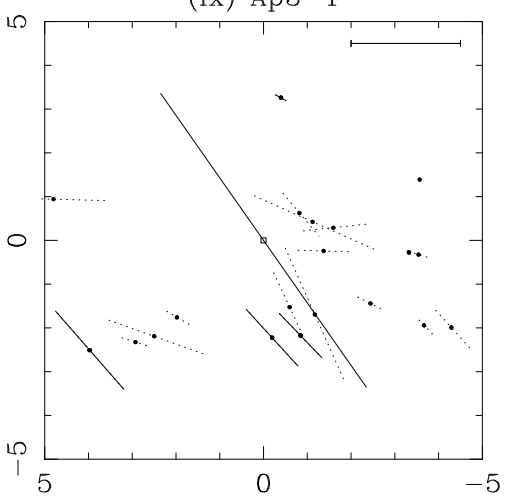

(xii) AS 281

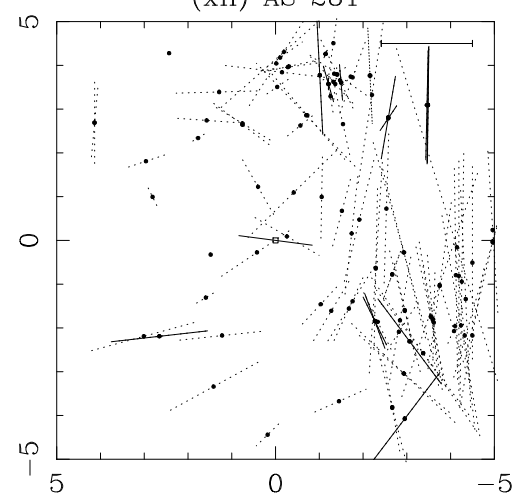

Fig. 1. Interstellar polarization maps of the regions surrounding the target stars. Dotted polarization vectors represent stars that are in the target area of sky but do not satisfy the distance criteria. The axes are in units of degrees and the continuum polarization of the target star is shown for comparison with the local ISP (open square). The line at the top right of each diagram represents $1 \%$ polarization 

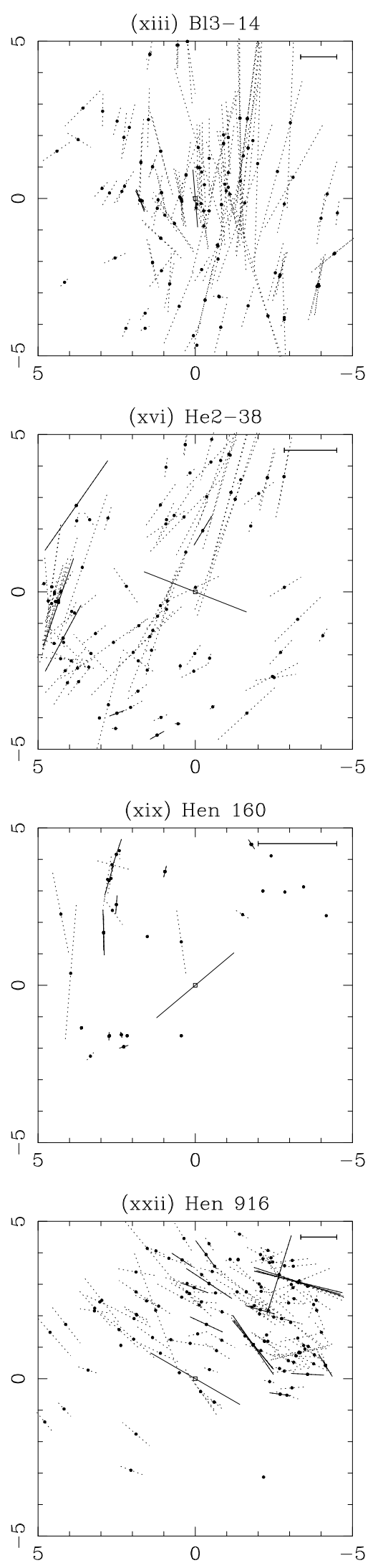

(xiv) $\mathrm{H} 2-5$
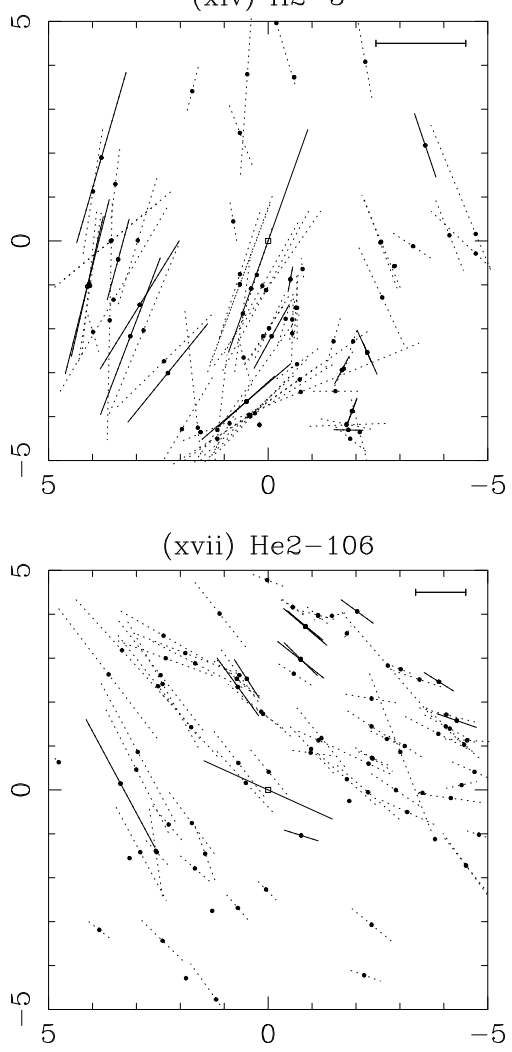

(xx) Hen 828

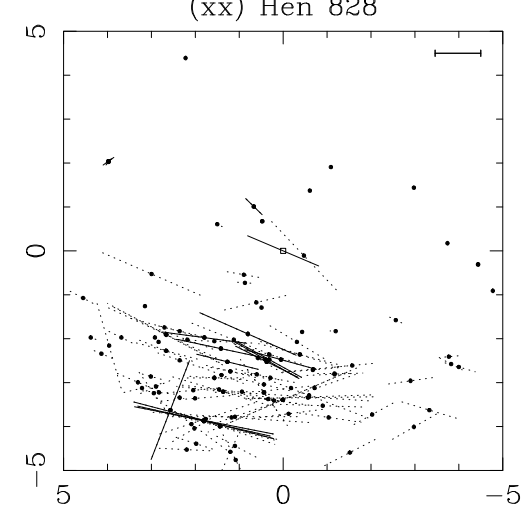

(xiii) Hen 1092

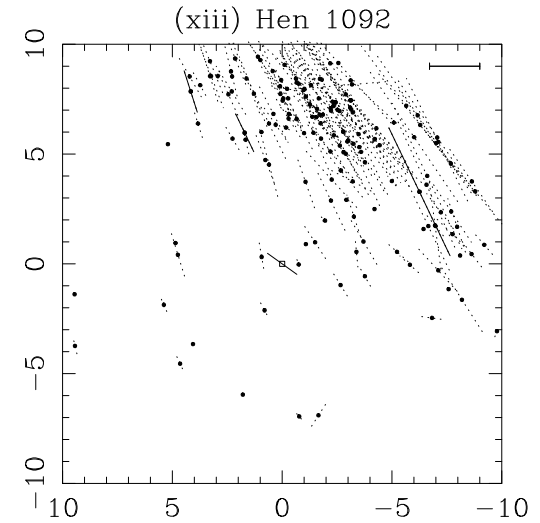

(xv) $\mathrm{H} 2-38$
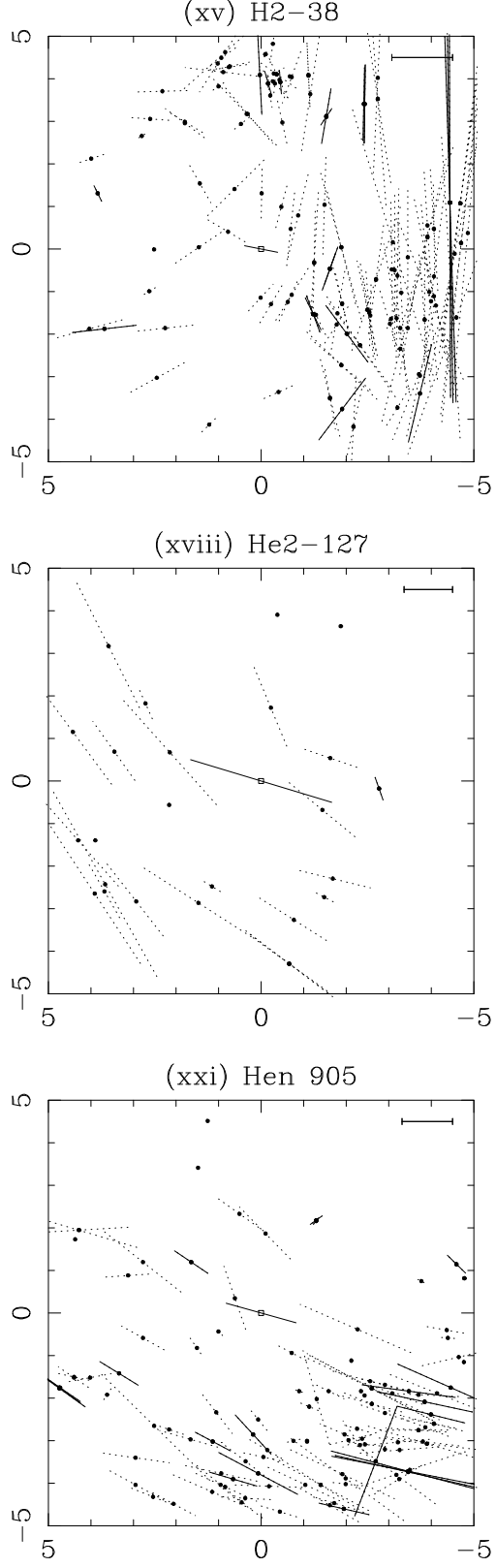

(xiv) Hen 1242

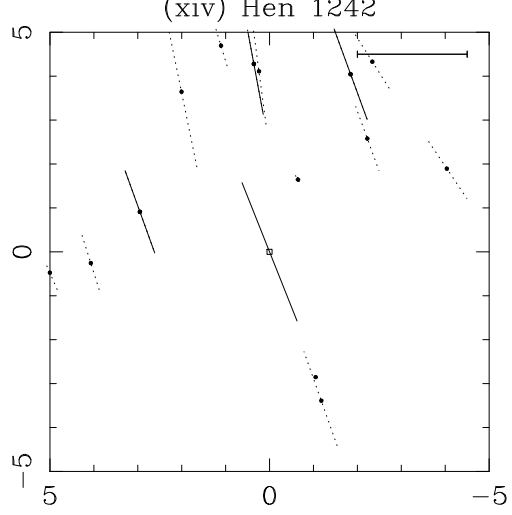

Fig. 1. continued 

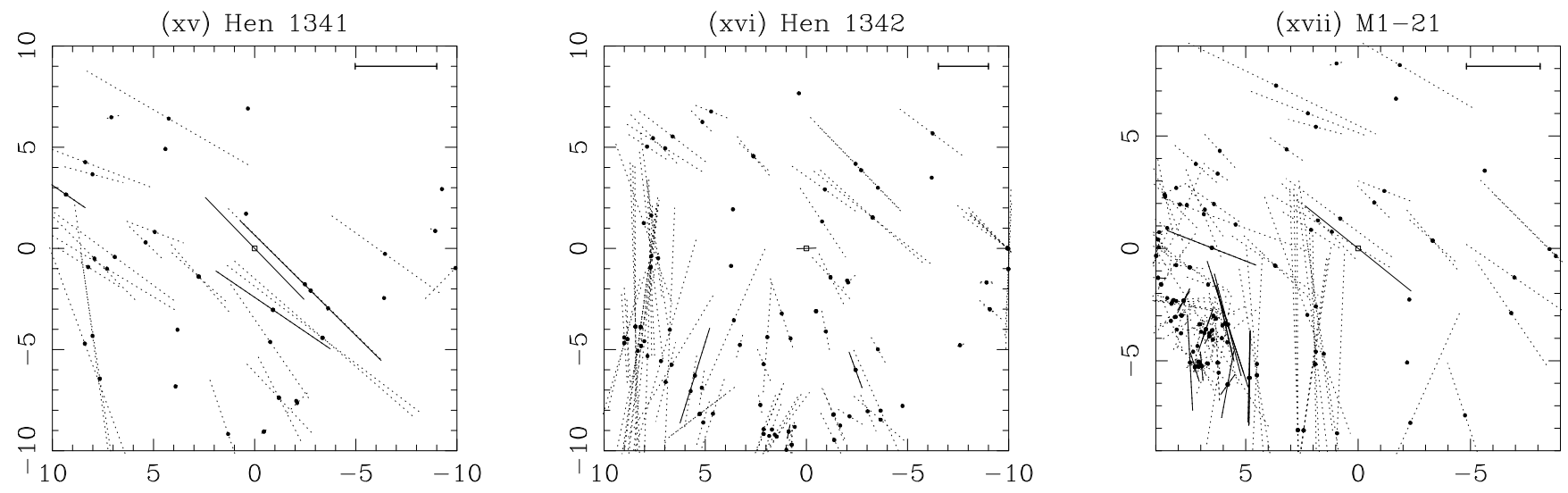

Fig. 1. concluded

in origin. It is possible that some of these stars do have an intrinsic polarization, but, until further observations are available which demonstrate either significant departures from a Serkowski law or variability, we must apply Occam's razor.

We summarize the results of this discussion, including, where appropriate, the adopted ISP vectors, in Table 3. The ISP wavelength dependence could be modelled by using the Serkowski law, but it can safely be regarded as constant over the small wavelength range covered by our observations.

Table 3. Interstellar polarizations determined for the target stars

\begin{tabular}{|c|c|c|c|c|}
\hline \multirow[t]{2}{*}{ Object } & \multirow[t]{2}{*}{ Var? } & \multirow[t]{2}{*}{ IS? } & \multicolumn{2}{|c|}{ ISP } \\
\hline & & & $\bar{q}_{\text {is }}(\%)$ & $\bar{u}_{\text {is }}(\%)$ \\
\hline$\overline{\text { SY Mus }}$ & $\mathrm{y}$ & $\mathrm{y}$ & \multicolumn{2}{|c|}{ continuum } \\
\hline RX Pup & $\mathrm{y}$ & $\mathrm{n}$ & +0.13 & +0.26 \\
\hline V455 Sco & $\mathrm{n}$ & $\mathrm{n}$ & +0.23 & +0.11 \\
\hline RR Tel & $\mathrm{y}$ & $\mathrm{n}$ & +0.36 & +0.06 \\
\hline Ap3-1 & $\mathrm{n}$ & $\mathrm{n}$ & +0.03 & +0.47 \\
\hline AS 210 & $\mathrm{y}$ & $\mathrm{y}$ & \multicolumn{2}{|c|}{ continuum } \\
\hline He2-38 & $\mathrm{n}$ & $\mathrm{n}$ & -0.14 & -1.26 \\
\hline He2-106 & $\mathrm{y}$ & $\mathrm{y}$ & \multicolumn{2}{|c|}{ continuum } \\
\hline Hen 160 & $\mathrm{n}$ & $\mathrm{n}$ & +0.11 & -0.04 \\
\hline Hen 1342 & $\mathrm{n}$ & $\mathrm{n}$ & +0.59 & -0.22 \\
\hline
\end{tabular}

Stars for which the entries would otherwise be ' $n$, y, continuum' are omitted from this table. The table shows the object name, whether or not the object shows documented polarization variability, and whether or not the intrinsic vector is consistent with the local ISP. Where the ISP has been estimated from the surrounding field stars the normalized ISP Stokes parameters are given; otherwise the continuum polarizations in Table 1 are adopted.

\section{Line properties}

\subsection{The line-polarization morphology}

The following general description of the lines is primarily based on $\lambda 6825$, since this is the stronger line, and usually has superior $\mathrm{S} / \mathrm{N}$. The polarization morphology is usually repeated in the $\lambda 7082$ line, however.

The observations fully resolve the Raman lines, and reveal a great deal of polarization structure, but with a number of characteristics common to several stars. In order to examine the velocity structure the observations were converted to 'Raman parent' velocity space. This conversion is performed by inverting the Raman-scattering wavelength increase. The wavelength of the Raman parent photon $\lambda_{\mathrm{p}}$ is given by

$$
\frac{1}{\lambda_{\mathrm{p}}}=\frac{1}{\lambda_{\mathrm{r}}}+\frac{1}{\lambda_{\mathrm{Ly} \alpha}}
$$

where $\lambda_{\mathrm{r}}$ is the Raman-scattered wavelength and $\lambda_{\mathrm{Ly} \alpha}$ is the wavelength of Ly $\alpha$. The Raman parent wavelength may then be converted to velocity space using the rest wavelength of the Raman parent (O VI). This conversion introduces a an improvement of a factor $\lambda_{\mathrm{p}} / \lambda_{\mathrm{r}} \simeq 7 \mathrm{in}$ the effective resolution of the spectra, so that the velocity resolution in 'parent' space is $\sim 8 \mathrm{~km} \mathrm{~s}^{-1}$.

\subsubsection{Intensity and polarization profiles}

All the intensity profiles show a multi-peaked structure, usually consisting of a strong central peak with adjacent weaker components. The bluewards peak is often strong (e.g., V455 Sco and RR Tel) and distinct from the central maximum, but sometimes is only evident as a 'shoulder' on the side of the intensity profile (e.g., AS 210 and Hen 160). The redward intensity peak varies in strength greatly from object to object, with some spectra showing a clear secondary maximum (e.g., He2-38), others displaying a red shoulder (e.g., Hen 916) while a smaller number show no clear evidence for a redwards component (e.g., RT Ser and AS 276). 
The polarization line-profiles minic the structure of the intensity profiles, in that they frequently have a triplepeaked structure (e.g., V455 Sco and M1-21) with the red peak usually of lowest polarization. The redwards polarization peak is sometimes observed as an extended polarized 'wing' (e.g., H2-38 and He2-38). Occasionally a single polarization peak is observed (e.g., AS 210). The number of measurable peaks in the polarization profile of each object is listed in Table 4 . There is no distinct correlation between the number of peaks and the cool-component spectral type. Both early- and late-type M systems can display triple-peaked structures, and similarly a range of subtypes have a single-peaked structure.

\subsubsection{Position-angle dependence}

Many stars show a polarization PA discontinuity, or 'flip', which is often, but not always, through $90^{\circ}$. This flip is most often observed in the triple-peaked polarization profiles, and usually occurs between the middle and red polarization peaks. Another common characteristic is a rotation of PA through the line. This rotation is most clearly seen in the $Q U$ plane, where the rotation leads to a $Q U$ loop (see Fig. 2).

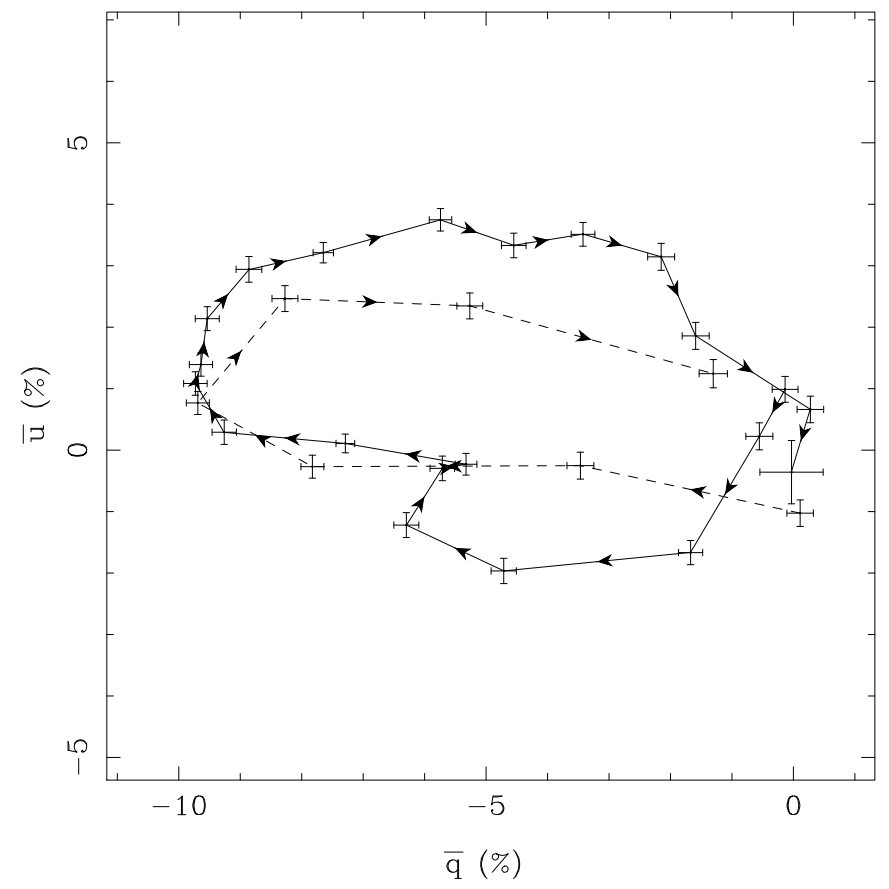

Fig. 2. The $Q U$ loop formed by the $\lambda 6825 \AA$ (solid line) and $\lambda 7082 \AA$ (dashed line) Raman lines of H2-38. The continuum polarization has been subtracted and the arrows show the blue-to-red direction

The gross properties of the lines are summarized in Table 4. The number of individual peaks in each profile and the position of the peak in Raman-parent velocity space, measured interactively by estimating the centroid of
Table 4. $\lambda 6825$ polarization-profile summary for the survey stars

\begin{tabular}{|c|c|c|c|c|}
\hline $\begin{array}{l}\text { Object } \\
\text { name }\end{array}$ & $\begin{array}{l}\text { No. of } \\
\text { peaks }\end{array}$ & $\begin{array}{l}\text { PA flip } \\
\text { (degrees) }\end{array}$ & $\begin{array}{c}\text { PA } \\
\text { rotation? }\end{array}$ & $\begin{array}{l}\text { Peak sep. } \\
\left(\mathrm{km} \mathrm{s}^{-1}\right)\end{array}$ \\
\hline$\overline{\text { SY Mus }}$ & 3 & 70 & & 48,64 \\
\hline V2506 Sgr & 2 & & yes & 54 \\
\hline HK Sco & 3 & & & 83,54 \\
\hline V455 Sco & 3 & 70 & & 51,70 \\
\hline RT Ser & 3 & & yes & 46,46 \\
\hline RR Tel & 3 & 90 & yes & 40,67 \\
\hline $\mathrm{CD}-43^{\circ} 14304$ & 2 & & yes & 19 \\
\hline Ap3-1 & $1 ?$ & & & \\
\hline AS 210 & 1 & & yes & \\
\hline AS 276 & 3 & & & 50,55 \\
\hline AS 281 & 3 & & yes & 59,48 \\
\hline $\mathrm{H} 2-5$ & $1 ?$ & & & \\
\hline $\mathrm{H} 2-38$ & 2 & & yes & 48 \\
\hline He2-38 & 3 & 90 & yes & 55,93 \\
\hline He2-106 & 2 & 90 & yes & 83 \\
\hline He2-127 & 2 & & yes & 45 \\
\hline Hen 160 & 1 & & yes & \\
\hline Hen 905 & 2 & 90 & yes & 199 \\
\hline Hen 916 & 2 & 50 & & 67 \\
\hline Hen 1092 & 2 & 60 & & 54 \\
\hline Hen 1242 & 3 & 55 & yes & 77,25 \\
\hline Hen 1341 & 1 & & & \\
\hline Hen 1342 & 2 & & & 41 \\
\hline M1-21 & 3 & 80 & yes & 42,77 \\
\hline
\end{tabular}

Poor S/N for Ap3-1 and H2-5. There is no measurable line polarization for RX Pup, Bl3-14, or Hen 828.

each peak, are listed, together with the velocity separation of the peaks. If a PA flip is present the amplitude was measured, otherwise the presence of any $\mathrm{PA}$ rotation is noted.

The velocity range of the polarization peaks shows a remarkably small range, with the majority of objects showing velocity separations of the order of $50 \mathrm{~km} \mathrm{~s}^{-1}$. The outstanding object is Hen 905, which shows a separation of $199 \mathrm{~km} \mathrm{~s}^{-1}$. In triple-peaked structures, the blue-middle separation is usually comparable to the middle-red separation.

\subsubsection{Comparison with Schmid \& Schild}

Schmid \& Schild (1994) carried out pioneering spectropolarimetry of symbiotic systems. Our observations broadly support most of their results, but they were handicapped by relatively poor signal-to-noise and, especially, by relatively poor resolution. We were also able to survey a larger number of stars. Thus whereas SS94 suggested that a PA flip may be only associated with symbiotic Miras and late M-types, our survey shows it to be a more widely distributed phenomenon, seen in the $\mathrm{K} /$ early-M systems 
Hen 905, Hen 1092, and M1-21, as well as D-type (Mira) systems such as RR Tel and He2-38.

Schmid \& Schild also proposed a categorization scheme for line-profile types on the basis of increasing polarization complexity. Our observations suggest that their scheme may have been premature. For example, several of the stars which they assign to their 'type I' (Raman lines with one component, relatively constant polarization, and no significant rotation), such as He2-38 and He2-127, show substantial structure in both the intensity and polarization profiles in our data. This is may in part be due to variability (see Sect. 5.6), but is likely to be due in part to differences in the nature of the data. With high-resolution observations it seems plausible that the basic structure is essentially triple-peaked (in both polarization and intensity), but with considerable variations in the relative strengths of the components (especially the redmost feature).

\subsection{The effect of the diluting continuum}

The continuum flux at the Raman-line wavelengths, although relatively weak, has a significant effect on the Raman-line polarization spectrum. In particular, it will, in general, dilute the polarization of the Raman lines, leading to a reduction in the observed polarization. Schmid \& Schild (1994) recognized this problem, and so performed a continuum subtraction on their data. We removed the polarized continuum from our data using

$$
I_{1}=I_{\mathrm{o}}-I_{\mathrm{c}} \quad Q_{\mathrm{l}}=Q_{\mathrm{o}}-Q_{\mathrm{c}} \quad U_{\mathrm{l}}=U_{\mathrm{o}}-U_{\mathrm{c}}
$$

where $\left(I_{\mathrm{o}}, Q_{\mathrm{o}}, U_{\mathrm{o}}\right)$ are the observed Stokes parameters and $\left(I_{1}, Q_{1}, U_{1}\right)$ are corresponding line Stokes parameters. The continuum polarization vector $\left(I_{\mathrm{c}}, Q_{\mathrm{c}}, U_{\mathrm{c}}\right)$ was obtained by summing the Stokes vectors over line-free continuum bins adjacent to the line. Figure 3 illustrates the effect of removing the continuum flux from one of our observations (cf. Fig. 9xxa).

The continuum-corrected data show a significant rise in polarization into the blue, as reported by SS94, with the bluemost data showing polarizations approaching $100 \%$. There is also evidence for a small rise in the red. However, the uncertainty in the polarization spectrum is large in the line wings, because line flux is small; also, at the very low signal:noise ratios in line wings, the effects of kurtosis on the probability distribution of $P$ become important, and $P$ is biased towards positive values. In addition, any error in the continuum placement can propagate into a large error in the continuum-subtracted polarization spectrum. This problem can be severe in symbiotic systems, where continuum placement can be difficult (see below). We therefore suggest that the continuum-subtracted polarized-flux spectrum may provide a more useful point of comparison with the models, as it is free of many of these difficulties.

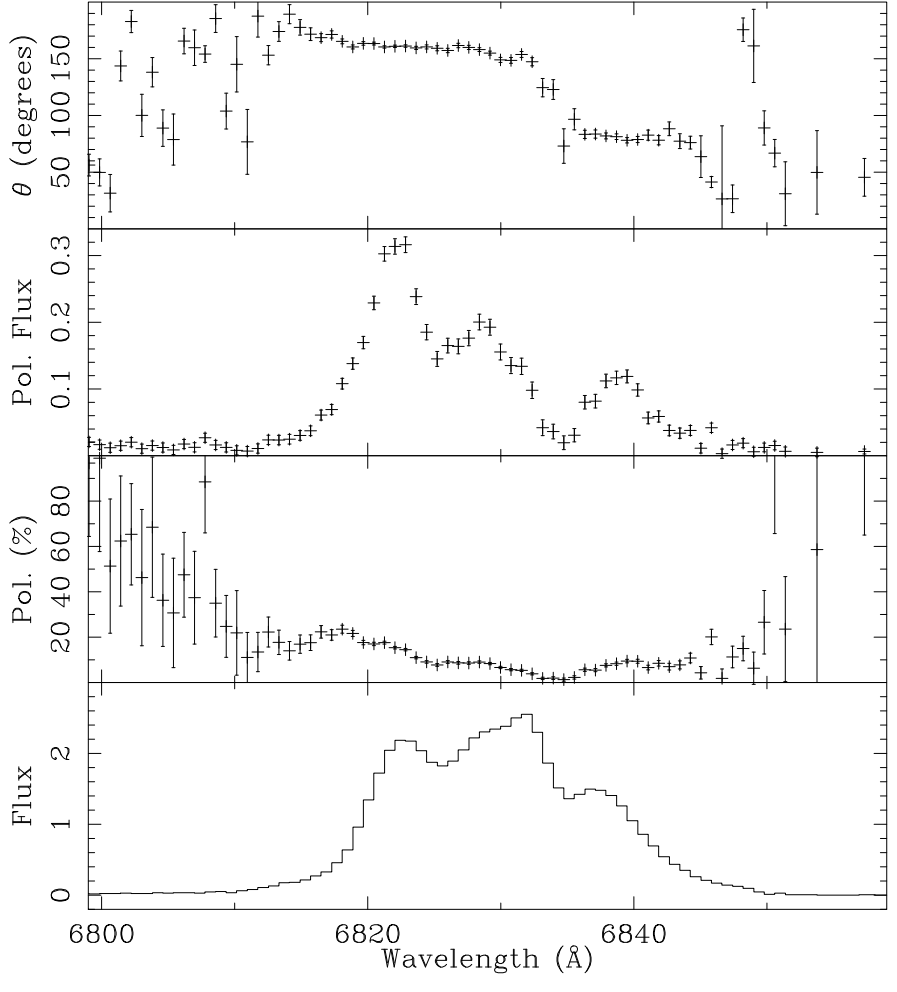

Fig. 3. The continuum-subtracted polarization spectrum of M1-21

\subsection{Equivalent-width measurements and spectral types}

The presence of molecular-absorption bands in the symbiotic spectra means that the equivalent widths of the Raman-scattered lines can be difficult to measure. To facilitate these measurements we synthesized the 'underlying' spectrum as a combination of a cool star plus a hotcomponent contribution (which may include continuous nebular emission). For the cool-star spectrum we observed numerous bright cool-giant standards, covering a range of spectral types (K5 to M7), while the hot component was assumed to have a flat spectral signature over the limited wavelength range observed.

If $I_{\mathrm{g}}$ is the normalized intensity of the cool component, then the modelled normalized symbiotic intensity may be written

$$
I_{\mathrm{s}}=\left(I_{\mathrm{g}}+C\right) /(1+C)
$$

where $C$ is the normalized intensity of the hot component. If $I_{\mathrm{O}}$ is the observed intensity, then $C$ (and the cool-star type) may be estimated by minimizing the function

$$
F=\int\left(I_{\mathrm{o}, \lambda}-I_{\mathrm{s}, \lambda}\right)^{2} \mathrm{~d} \lambda
$$

The fit was performed over regions of the symbiotic spectrum not contaminated by emission lines or telluric absorption, with small wavelength shifts applied to the 
standard-star spectra where necessary, to account for differing radial velocities. Two examples are shown in Fig. 4, and the spectral types and $C$ factors are reported in Table 5 .

The agreement between the synthesized and observed spectra is generally good, and the inferred cool-star spectral types are close to published values in most cases. The continua of RX Pup, He2-38, and He2-106 could not be successfully fit by this method; fortunately, the spectra of these objects are relatively featureless in the region of the Raman lines, and so it was possible to rectify the lines using a simple low-order polynomial fit to the continuum. For three stars (He2-127, Hen 905, Hen 1092) the differences between our the $2-\mu \mathrm{m}$ spectral types reported by Allen (1980b) and our estimates are substantial; in each case we rated our fit as relatively poor, but adopting Allen's types would give significantly worse results in our spectra. Since our principal concern was to measure reliably the Raman-line strengths, we retained our values.

The fits were used to rectify the spectra, and the equivalent widths of the Raman lines then measured interactively using the DIPSO spectrum analysis package (Howarth \& Murray 1991). The results are given in Table 5 . In general, the D-type systems show the largest equivalent widths; the cool (Mira-type) components in these systems are expected to large mass-loss rates, and hence large Raman-scattering optical depths.

A plot of equivalent width (EW) of the $6825 \AA$ line versus the EW of the $7082 \AA$ line is shown in Fig. 5 . This plot demonstrates a well-defined, approximately linear relationship between the equivalent widths of the two lines, as previously demonstrated by Allen (1980a). An unweighted linear least-squares fit to our data yields

$$
\operatorname{EW}(6825)=4.36( \pm 0.16) \operatorname{EW}(7082)+6.0( \pm 4.0)
$$

with a linear correlation coefficient of 0.98. (A non-zero intercept was allowed because only the $6825 \AA$ line is detectable in some systems.) This result is in satisfactory agreement with the fit given by Allen (1980a):

$$
\mathrm{EW}(6825)=5.5( \pm 0.6) \mathrm{EW}(7082)
$$

although the fits deviate at large equivalent widths. This may be due to systematic errors in one or both sets of EW measurements.

\subsection{Polarization measurements}

We can define a line-polarization parameter as

$$
\eta=\frac{\int \sqrt{\left(Q_{\lambda}(l)-Q_{\lambda}(c)\right)^{2}+\left(U_{\lambda}(l)-U_{\lambda}(c)\right)^{2}} \mathrm{~d} \lambda}{\int I_{\lambda}(l)-I_{\lambda}(c) \mathrm{d} \lambda}
$$

where the $l$ and $c$ qualifiers refer to the line flux and continuum, respectively. The $\eta$ parameter is the ratio of the polarized line-flux to the total line-flux; that is, if (a) SY Mus

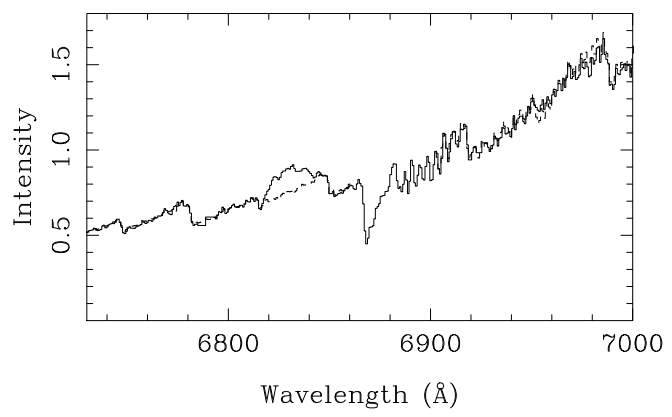

(b) AS 276

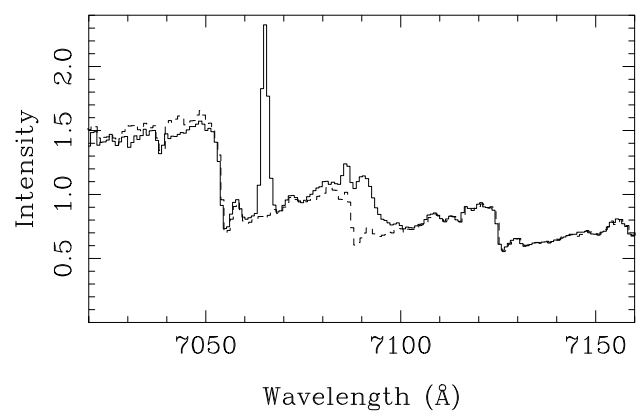

Fig. 4. An example of the fit to the underlying spectrum of the symbiotic stars a) SY Mus and b) AS 276. The solid line is the observation and the dashed line is the synthesized spectrum

the line flux is regarded as a mix of a $100 \%$ linearly polarized beam and a completely unpolarized beam, then $\eta=I(100) /(I(100)+I(0))$.

A plot of $\eta_{7082}$ against $\eta_{6825}$ is given in Fig. 6. A Spearman's rank-order analysis of the data yields a correlationcoefficient of 0.75 , which differs from zero with a $99 \%$ confidence. A linear least-squares fit to these data yield a gradient of $0.9 \pm 0.2$, implying that there is no measurably significant difference in the scattering properties that result in the $\lambda 6825$ and $\lambda 7082$ lines. This is a slightly surprising result, since the smaller cross-section of the $\lambda 1038 \rightarrow \lambda 7082$ transition compared to $\lambda 1032 \rightarrow \lambda 6825$ leads to the expectation that the $\lambda 7082$ line may be produced in a different scattering volume, due to the greater mean free path of the $\lambda 1038$ photons.

A plot of $\eta_{6825}$ against cool-component spectral type (Fig. 7) shows no clear correlation. In particular, the Dtype systems, with largest Raman-line strengths, do not show systematically high values of $\eta$. The large scatter in Fig. 7 suggests that the Raman-line polarization is not a simple function of mass-loss rate, which we expect to correlate loosely with spectral type, but may be more strongly dependent on other factors (such as viewing angle of the binary system). A plot of $\eta_{6827}$ against $K-$ [12] colour (which is a more direct indicator of mass-loss rate; 
Table 5. The equivalent widths, $\eta$ parameters, and velocities of the Raman-scattered lines. The estimated M-giant spectral type and $C$ factor (Eq. 23) are given where fits were made to the symbiotic continuum. Gaps in the equivalent-width columns correspond to polarization spectra in which the Raman line was undetectable. Errors on the EW were obtained by repeated measurements

\begin{tabular}{|c|c|c|c|c|c|c|c|c|}
\hline \multirow[b]{2}{*}{ Object } & \multicolumn{2}{|l|}{ Fit } & \multicolumn{2}{|c|}{ EW $(\AA)$} & \multicolumn{2}{|c|}{$\eta$} & \multicolumn{2}{|c|}{$\bar{v}(\mathrm{~km} / \mathrm{s})$} \\
\hline & Туре & $C$ & $\lambda 6825$ & $\lambda 7082$ & $\lambda 6825$ & $\lambda 7082$ & $\lambda 6825$ & $\lambda 7082$ \\
\hline SY Mus & M5.5 & 0.32 & $4 \pm 1$ & $2 \pm 1$ & 0.079 & 0.054 & 259 & 170 \\
\hline RX Pup & & & $0.15 \pm .05$ & $\ldots$ & - & $\ldots$ & 85 & $\ldots$ \\
\hline V2506 Sgr & M5.5 & 0.47 & $23 \pm 1$ & $5 \pm 1$ & 0.080 & 0.076 & 166 & 220 \\
\hline HK Sco & M2 & 0.04 & $19 \pm 1$ & $4 \pm 1$ & 0.031 & 0.033 & 440 & 476 \\
\hline V455 Sco & M5.5 & 0.77 & $43 \pm 1$ & $6 \pm 1$ & 0.110 & 0.095 & 154 & 250 \\
\hline RT Ser & M7 & 0.50 & $37 \pm 1$ & $9 \pm 1$ & 0.060 & 0.060 & 215 & 182 \\
\hline RR Tel & M5.5 & 1.58 & $124 \pm 2$ & $21 \pm 1$ & 0.094 & 0.081 & 111 & 162 \\
\hline $\mathrm{CD}-43^{\circ} 14304$ & M0 & 0.78 & $13 \pm 1$ & $5 \pm 1$ & 0.076 & 0.094 & 269 & 298 \\
\hline Ap3-1 & M7 & 0.23 & $6 \pm 1$ & $\ldots$ & $0.27:$ & $\ldots$ & 410 & $\ldots$ \\
\hline AS 210 & K5 & 0.98 & $24 \pm 2$ & $5 \pm 1$ & 0.071 & 0.093 & 261 & 182 \\
\hline AS 276 & M4 & 0.37 & $15 \pm 1$ & $5 \pm 1$ & 0.095 & 0.145 & 237 & 249 \\
\hline AS 281 & M5.5 & 0.44 & $22 \pm 1$ & $5 \pm 1$ & 0.094 & 0.066 & 193 & 215 \\
\hline Bl3-14 & & & & $\ldots$ & $\ldots$ & $\ldots$ & $\ldots$ & $\ldots$ \\
\hline $\mathrm{H} 2-5$ & M7 & 0.13 & $15 \pm 1$ & $4 \pm 1$ & $0.15:$ & $0.20:$ & 195 & 294 \\
\hline H2-38 & M2 & 1.70 & $333 \pm 15$ & $75 \pm 3$ & 0.075 & 0.093 & 252 & 277 \\
\hline He2-38 & & & $123 \pm 5$ & $14 \pm 1$ & 0.036 & 0.031 & 269 & 269 \\
\hline He2-106 & & & $293 \pm 36$ & $63 \pm 1$ & 0.024 & 0.022 & 248 & 255 \\
\hline He2-127 & K5 & 0.91 & $227 \pm 11$ & $52 \pm 3$ & 0.046 & 0.054 & 190 & 237 \\
\hline Hen 160 & M4 & 0.36 & $176 \pm 2$ & $47 \pm 1$ & 0.017 & 0.030 & 222 & 265 \\
\hline Hen 828 & M5.5 & 2.10 & $\ldots$ & $\ldots$ & $\ldots$ & $\ldots$ & $\ldots$ & $\ldots$ \\
\hline Hen 905 & M2 & 0.15 & $45 \pm 1$ & $7 \pm 1$ & 0.022 & 0.022 & 269 & 375 \\
\hline Hen 916 & M5.5 & 0.75 & $25 \pm 1$ & $6 \pm 2$ & 0.041 & 0.057 & 168 & 217 \\
\hline Hen 1092 & M5.5 & 0.35 & $13 \pm 1$ & $3 \pm 1$ & 0.071 & 0.097 & 314 & 244 \\
\hline Hen 1242 & M5.5 & 1.19 & $50 \pm 1$ & $9 \pm 1$ & 0.050 & 0.047 & 327 & 410 \\
\hline Hen 1341 & M2 & 0.16 & $9 \pm 1$ & $\ldots$ & 0.046 & $\ldots$ & 380 & $\ldots$ \\
\hline Hen 1342 & M0 & 0.76 & $10 \pm 1$ & $1 \pm 1$ & 0.040 & 0.062 & 158 & 268 \\
\hline M1-21 & M4 & 0.21 & $39 \pm 1$ & $8 \pm 1$ & 0.088 & 0.055 & 163 & 321 \\
\hline
\end{tabular}

e.g., Whitelock et al. 1994), using data from Whitelock \& Munari (1992), confirms this result.

\subsection{Velocity measurements}

Our observations were not designed for accurate radialvelocity measurements, nor is it straightforward to decide on what is 'the' velocity of a feature with complex morphology. To overcome these problems we have measured the differential velocities of the Raman lines with respect to the nebular He I 6678.15, $7065.19 \AA$ lines, using the first moment of the line profile to determine the wavelengths:

$$
\bar{\lambda}=\frac{\int(I-I(c)) \lambda \mathrm{d} \lambda}{\int(I-I(c)) \mathrm{d} \lambda} .
$$

The results of these measurements are reported in Table 5 . The difference in measured velocities for the helium lines averages $-4.1 \pm 18.3 \mathrm{~km} \mathrm{~s}^{-1}$ (s.d.). Since the magnitude of any orbital motions is unlikely to exceed $\sim 10 \mathrm{~km} \mathrm{~s}^{-1}$ (i.e., smaller than our measurement uncertainties), the differ- ential velocities can be interpreted directly in terms of the dynamics of the line formation.

The most striking result is that all the Raman lines show an overall redshift from the expected rest wavelength, typically by $200-300 \mathrm{~km} \mathrm{~s}^{-1}$ (in 'observed' space) but by more than $400 \mathrm{~km} \mathrm{~s}^{-1}$ for HK Sco. There is no systematic difference between the $\lambda 6825 \AA$ and $\lambda 7082 \AA$ velocities, although the scatter is large (principally because of the general weakness and difficulty of continuum placement for the $\lambda 7082 \AA$ line). The implication is that there is a nett velocity of recession between the source of parent $\mathrm{O}$ VI photons and the neutral-hydrogen scattering region.

\subsection{Temporal variations}

In a short exploratory programme, five of the symbiotic systems observed in the May 1994 survey were first observed in March 1992, using the same equipment. (Seven of our targets were also observed in June/July 1992 by SS94, but the factor $\sim 6$ difference in resolutions 


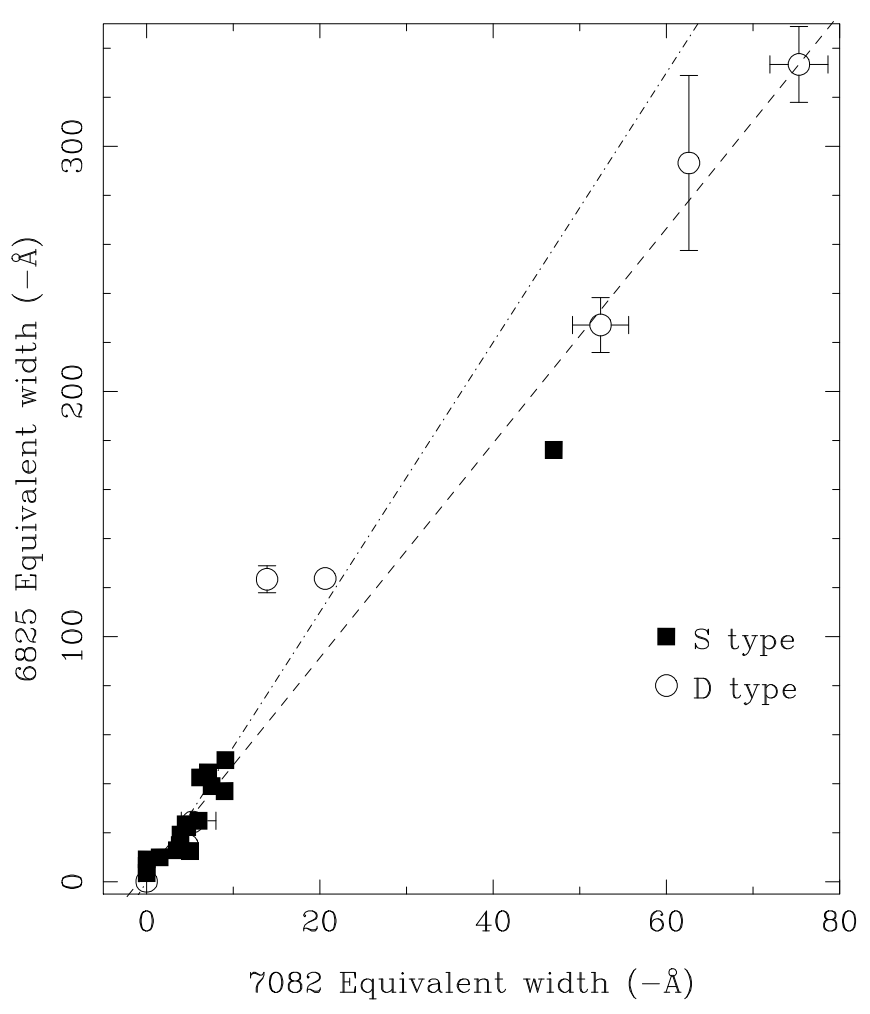

Fig. 5. The equivalent widths of the Raman-scattered lines. Solid squares represent S-type systems, and open circles D-type systems. The least-squares straight-line fit to these data is shown (Eq. (25), dashed line) along with the fit given by Allen (1980a) (Eq. (26), dot-dashed line)

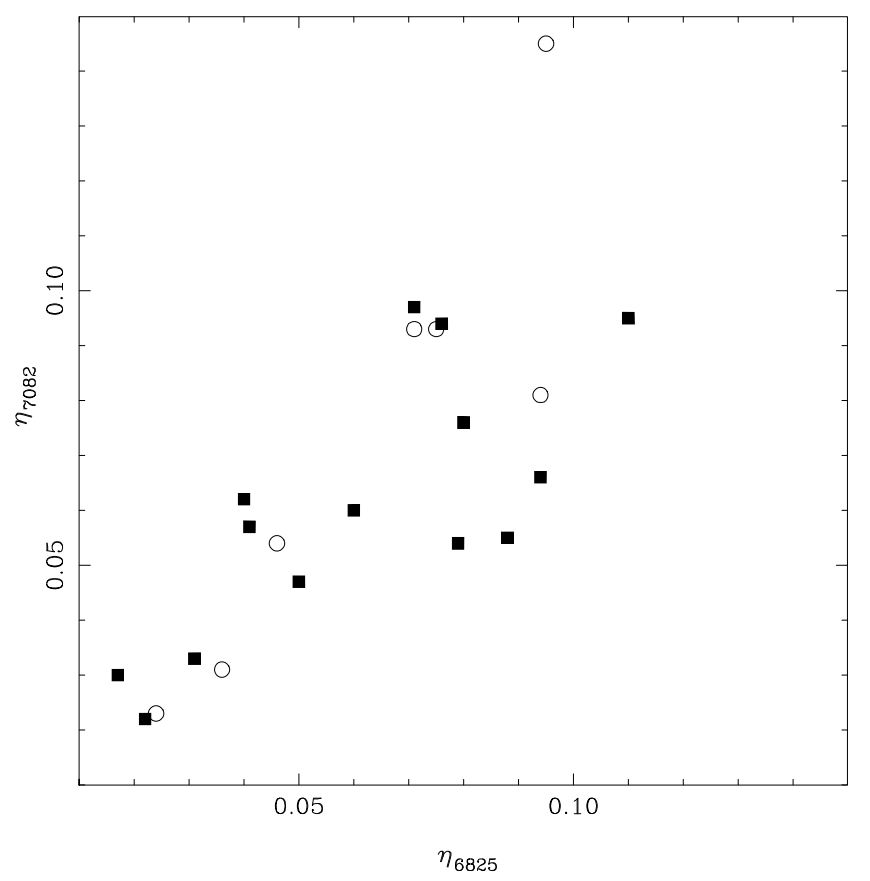

Fig. 6. A plot of $\eta_{7082}$ versus $\eta_{6825}$ for the symbiotic stars; filled squares represent S-type systems, and open circles D-type systems

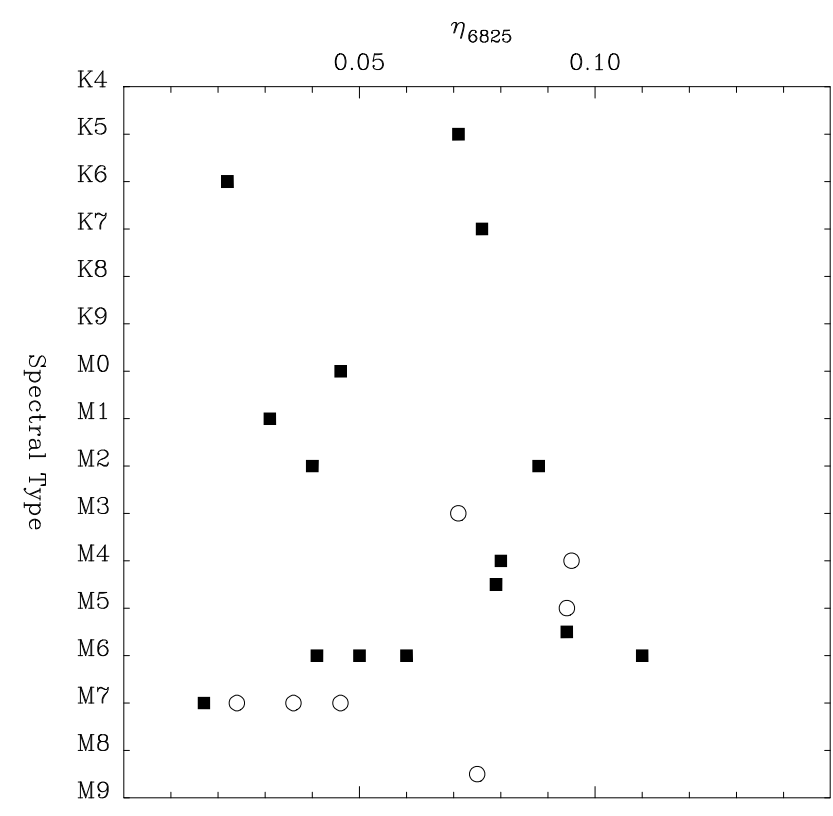

Fig. 7. A plot of $\eta_{6825}$ versus spectral type of the cool component (symbols as for Fig. 6)

renders comparisons of doubtful value.) The 1992 and 1994

observations of four of these stars are compared in Fig. 8. The fifth object, SY Mus, has been observed in two further observing campaigns (April and August 1995) and the data are presented elsewhere (Harries \& Howarth 1996a).

(i) He2-106: This D-type system showed an increase in the magnitude of polarization in the blue peak between the two observations. A PA rotation is present at both epochs, and is very similar through the blue side of the profile but continues through the red side of the profile in the 1992 observation.

(ii) Hen 1242: This M6 S-type system shows a dramatic change in the magnitude of the line polarization, with a blueward peak in 1992 that is much weaker in the 1994, and an enhanced redward peak in 1994. The red wing of the polarization profile remains unchanged. The PA structure of this system also changes between observations, while the rectified intensity is essentially constant.

(iii) He2-38: Another D-type binary, this system has a basically single-peaked polarization structure in the 1992 observation but a more complicated, triple-peaked profile in the 1994 observation. The PA also shows a significant change, while the rectified intensity hardly varies. Our 1992 spectrum was obtained $\sim 100 \mathrm{~d}$ before the observation reported by SS94, is broadly consistent with their data, although showing more structure (especially in the intensity profile). 
(iv) RR Tel: This third D-type system shows very similar polarizations in the 1992 and 1994 observations, with only a slight decrease in polarization magnitude and polarized profile width. The intensity profile shows a significant variation.

Overall, therefore, the rectified intensity profiles of the Raman lines in these data appear to be very stable, with only RR Tel showing a substantial change. RR Tel is well known to show large-amplitude changes in brightness associated with its Mira-type variability; in this case, therefore, the change in (normalized) line intensity may be a reflex response to a change in continuum level. Although such a change in the continuum flux will lead to a change in the observed line polarization (as the intensity of diluting radiation changes) the strength of the Raman lines in RR Tel means that this polarization change will be negligible. The stability of the intensity profiles and continuum polarization belies the marked changes that occurred in the polarization profiles between 1992 and 1994, however.

\section{Discussion}

Of the 27 stars surveyed, 25 show the $\lambda 6825 \AA$ Raman line. The exceptions are Hen 828 and Bl3-14; for these systems our data quality is such that we should certainly have detected any previously-observed feature. In each case the presence of He I emission lines ( $\lambda \lambda 6678,7065 \AA)$ confirms that the correct target was observed, and we conclude that the Raman lines have diminished in strength since Allen's (1984) observations. In support of this interpretation, Gutiérrez-Moreno \& Moreno (1995) document recent extensive spectroscopic variability in Bl3-14.

Twenty-four of the 25 systems showing $\lambda 6825 \AA$ also show significant polarization in the line. The exception is RX Pup, which has by far the smallest measured equivalent width. For two additional stars (Ap3-1 and H2-5) the data are such that the line polarizations are best regarded as detections, rather than measurements. We conclude that it is probable that sufficiently sensitive observations will always reveal polarization in the Raman lines.

We have measured the continuum $(\sim R$-band $)$ polarization for all targets. In most cases the measurements are consistent with the (often uncertain) foreground interstellar polarization, but seven stars show evidence for an intrinsic component. Furthermore, five (out of eight) stars with previous photopolarimetry show polarimetric variability, again indicating an intrinsic component. With two stars in common to the two lists, there are therefore ten systems in our sample which show evidence of intrinsic continuum polarization.

One of the principal goals of our survey was to define the broad characteristics of the Raman lines, in order to test numerical models of line formation and to explore the diagnostic potential of those models (Harries \& Howarth 1996b). We find the following: (i) For the 22 'well-observed' targets the line polarizations are quite large (up to $\sim 10 \%$ ). The strongest and most highly polarized lines are found in D-type (Mira) systems, but a measure of scattering efficiency (Eq. 5.4) shows no correlation with spectral type of the cool component.

(ii) The intensity and polarization morphologies are often complex in nature, with multi-peaked polarization profiles observed across a broad range of spectral types. The commonest structure is a triple-peaked line.

(iii) The Raman lines show a systematic redshift with respect to the expected rest wavelength, typically by $200-300 \mathrm{~km} \mathrm{~s}^{-1}$ (observed), while the peak-to-peak separations of multiple polarization peaks in the $\lambda 6825$ lines cluster around $\sim 50 \mathrm{~km} \mathrm{~s}^{-1}$ (in the 'parent velocity' system).

(iv) A PA flip, or discontinuity, is observed in about half the stars observed. This flip is often (though not always) $90^{\circ}$. Many of the objects display a PA rotation; there is no obvious relationship between the presence of PA rotation and spectral type, although only about half the S-type systems $(8 / 17)$, while most of the D-types $(6 / 7)$, show a rotation.

(v) Repeat observations of 5 symbiotic stars, separated by $\sim 750$ days, show relatively stable intensity profiles for the Raman lines, but significant changes in both magnitude and direction of the polarization.

The observations presented here clearly demonstrate that, in the majority of systems, scattering occurs in an extended, expanding wind. The formation of the Ramanscattered lines is a complex process involving a combination of factors, such as binary phase, mass-loss rate, velocity fields, and strength of the parent lines. We have already demonstrated the value of spectropolarimetry of the Raman lines in the determination of orbital parameters (Harries \& Howarth 1996a), but in order to use the observations as a diagnostic of other variables it is necessary to construct a model and examine the sensitivity of the Raman lines to changes in the system parameters. These calculations are reported in a companion paper (Harries \& Howarth 1996b; see also Harries 1995).

Finally, if the broad details of the scattering geometry are governed by mass loss from the red-giant component, then the velocity separation of the blue and red polarization peaks is directly related to the speed of the cool wind in the Raman-scattering region. Although we defer detailed discussion to an accompanying paper (Harries \& Howarth 1996b), we note here that the observed velocity separations are difficult to reconcile with the canonical low value for cool-wind expansion of $\sim 10 \mathrm{~km} \mathrm{~s}^{-1}$.

Acknowledgements. This work is based on observations obtained using the Anglo-Australian Telescope, Siding Spring, NSW and we would like to thank the AAO staff for their support. The computational work was performed on the UCL node 
(a)

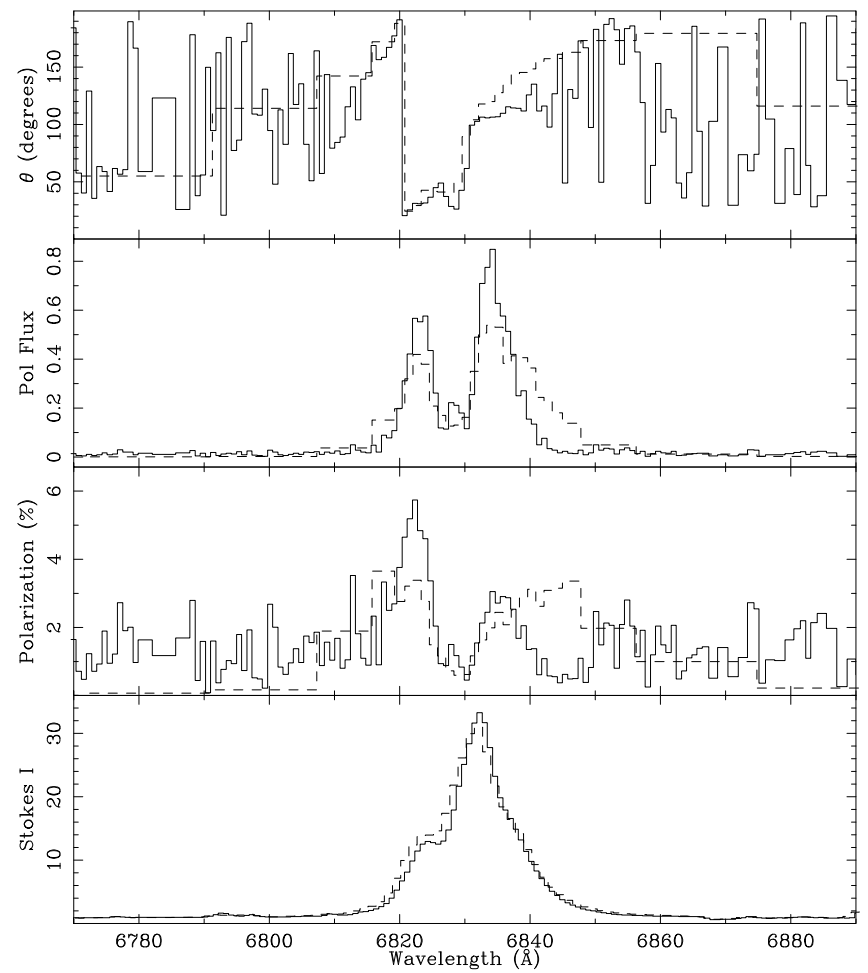

(c)

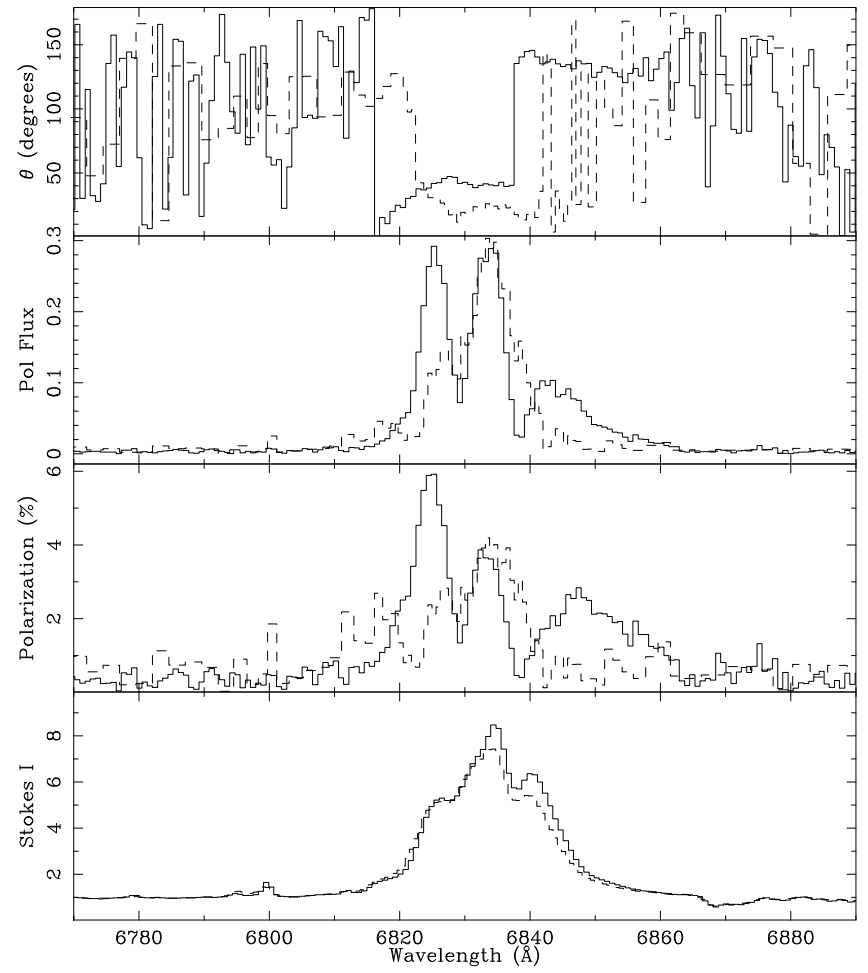

(b)

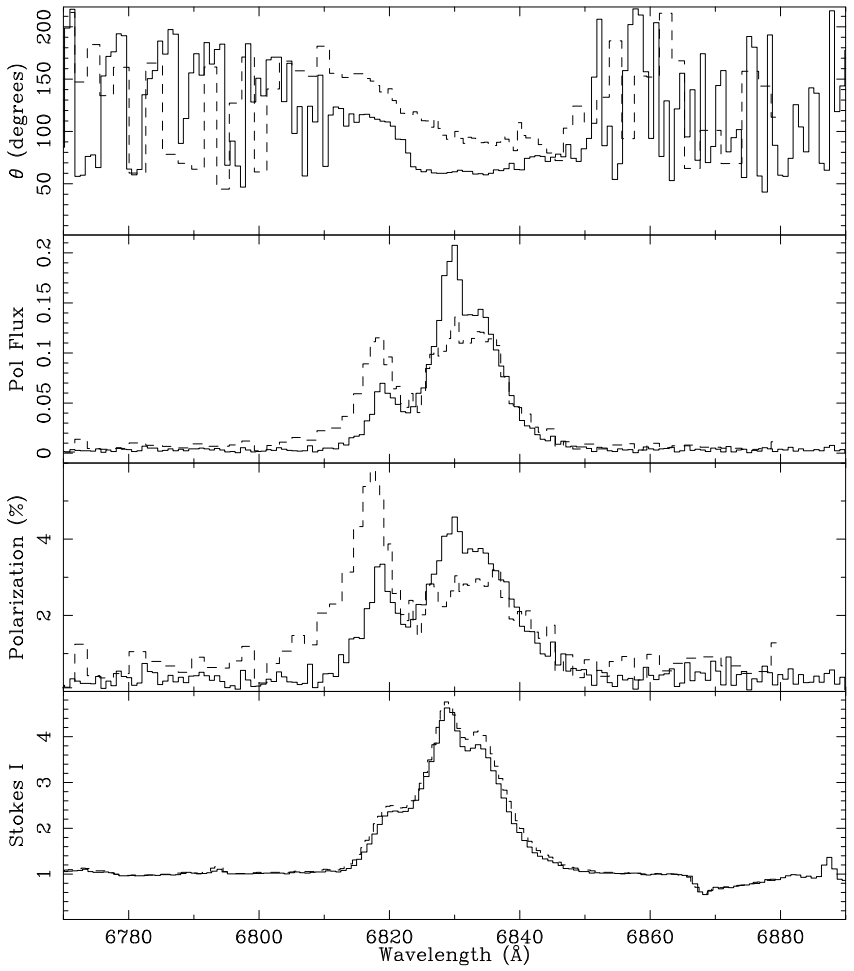

(d)

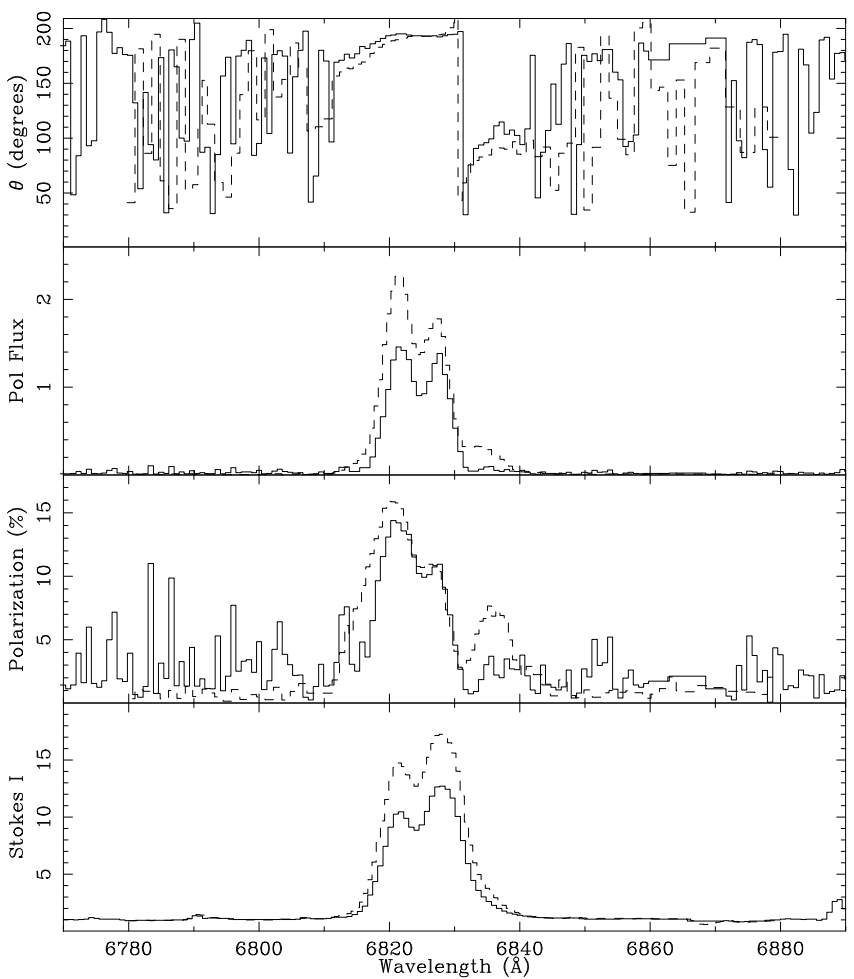

Fig. 8. a)-d). Plots of the $\lambda 6825 \AA$ line in the symbiotics stars a) He2-106, b) Hen 1242 , c) He2-38 and d) RR Tel. Each plot shows two observations: May 1994 (solid line) and March 1992 (dashed line). The polarization spectra have the continuum vector subtracted 
of the Starlink Project. TJH acknowledges a studentship from SERC/PPARC.

\section{References}

Allen C.W., 1973, Astrophysical Quantities (3rd edition). Athlone Press

Allen D.A., 1980a, MNRAS 190, 75

Allen D.A., 1980b, MNRAS 192, 521

Allen D.A., 1981, The Nature of Symbiotic Stars (IAU

Coll. 70). In: Friedjung M. \& Viotti R. (eds.). Kluwer, p. 27

Allen D.A., 1984, Proc. Aus. Astr. Soc. 5, 369

Clarke D., Stewart B.G., 1986, Vistas Astron. 29, 27

Crawford D.L., 1975, AJ 80, 955

Crawford D.L., 1978, AJ 83, 48

Crawford D.L., 1979, AJ 88, 1858

Gutiérrez-Moreno A., Moreno H., 1995, PASP 107, 541

Harries T.J., 1995, PhD Thesis, University of London

Harries T.J., Howarth I.D., 1996a, A\&A 310, 235

Harries T.J., Howarth I.D., 1996b, A\&AS (in press)

Hauck B., Mermilliod J.C., 1990, A\&AS 86, 107

Heintze J.R.W., 1973, in IAU Symp. 54 (Reidel), 231

Howarth I.D., Murray J., 1991, Starlink User Note No. 50

Kenyon S.J., 1986, The Symbiotic Stars, CUP

Kenyon S.J., Fernandez-Castro T., 1987, AJ 93, 938
Klare G., Neckel T., 1977, A\&AS 27, 215

Mathewson D.S., Ford V.I., Klare G., Neckel T., Krautter J., 1978, Bull. CDS 14, 115

Medina Tanco G.A., Steiner J.E., 1995, AJ 109, 1770

Mermilliod J.C., 1987, A\&AS 71, 413

Meyerdicks H., 1982, Starlink User Note No. 86

Moon T.T., 1985, Comms. Uni. Lon. Obs., 78

Munari U., Yudin B.F., Taranova O.G., et al., 1992, A\&AS 93, 383

Schmid H.M., 1989, A\&A 221, L31

Schmid H.M., Schild H., 1990, A\&A 236, L13

Schmid H.M., Schild H., 1994, A\&A 281, 145

Schmutz W., Schild H., Mürset U., Schmid H.M., 1994, A\&A 288,819

Schulte-Ladbeck R.E., 1988, A\&A 189, 97

Schulte-Ladbeck R.E., Aspin C., Magalhães A.M., Schwartz H.E., 1990, A\&AS 86, 227

Schulte-Ladbeck R.E., Magalhães A.M., 1987, A\&AS 181, 213

Whitelock P.A., 1987, PASP 99, 573

Whitelock P.A., 1998, in The Symbiotic Phenomenon (IAU Coll. 103). In: Mikolajewska J. et al. (eds.), p. 47

Whitelock P.A., Munari U., 1992, A\&A 255, 171

Whitelock P.A., et al., 1994, MNRAS 267, 711

\section{A. The spectropolarimetric data}


(ia) SY Mus $6825 \AA$

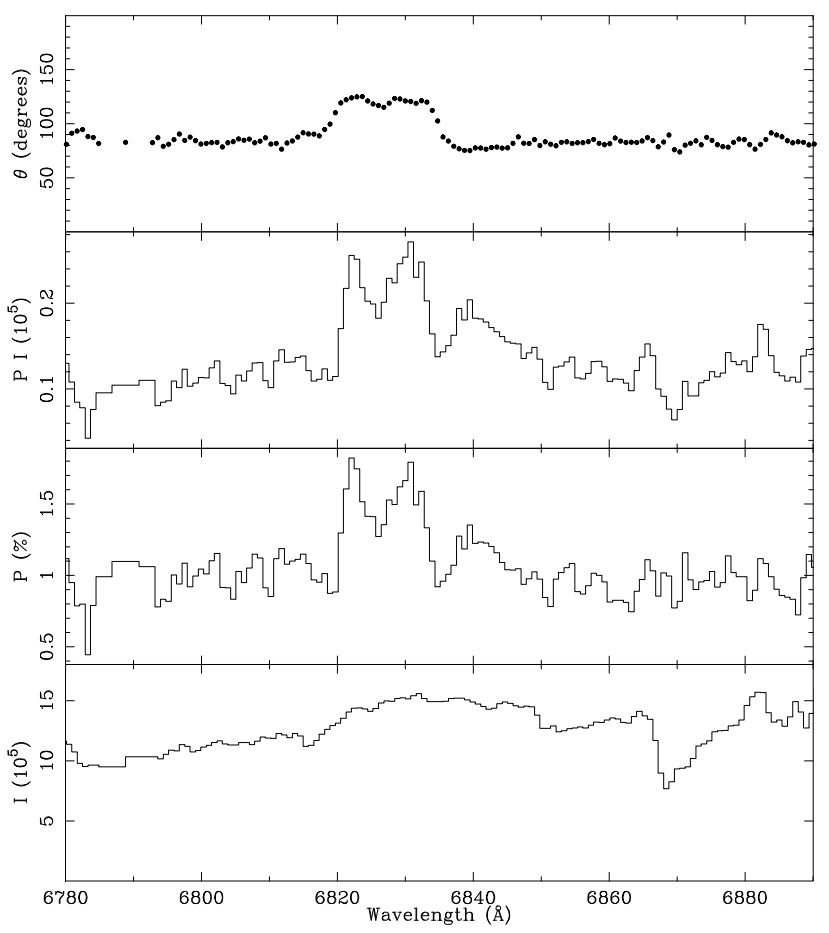

(iia) RX Pup $6825 \AA$

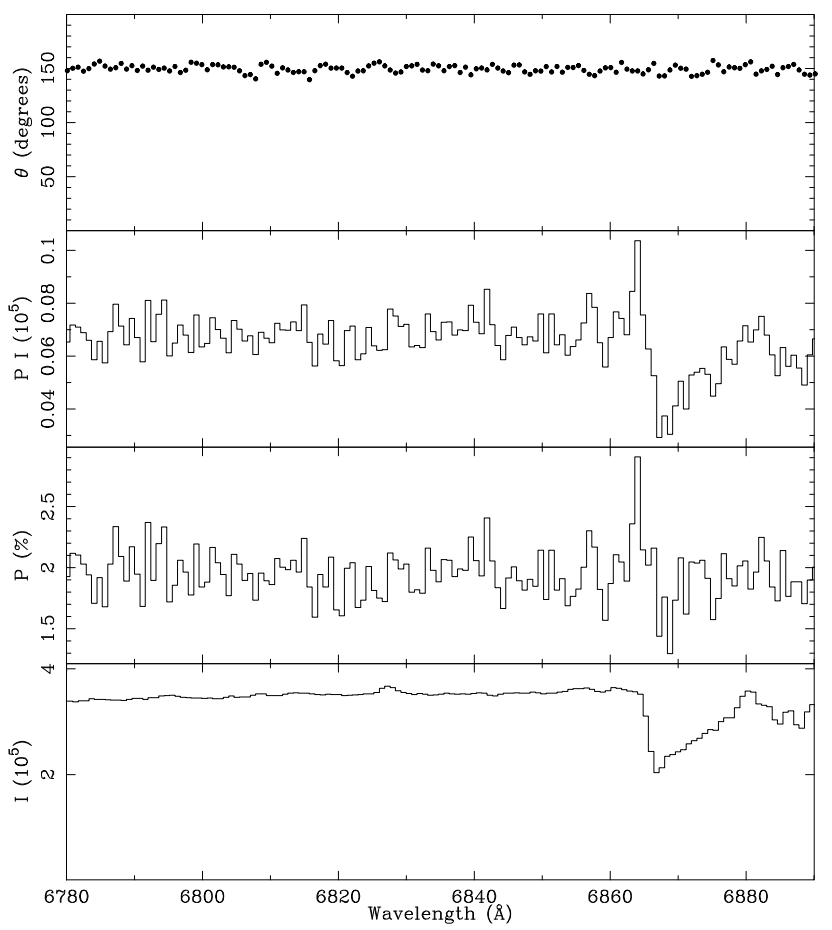

(ib) SY Mus 7082

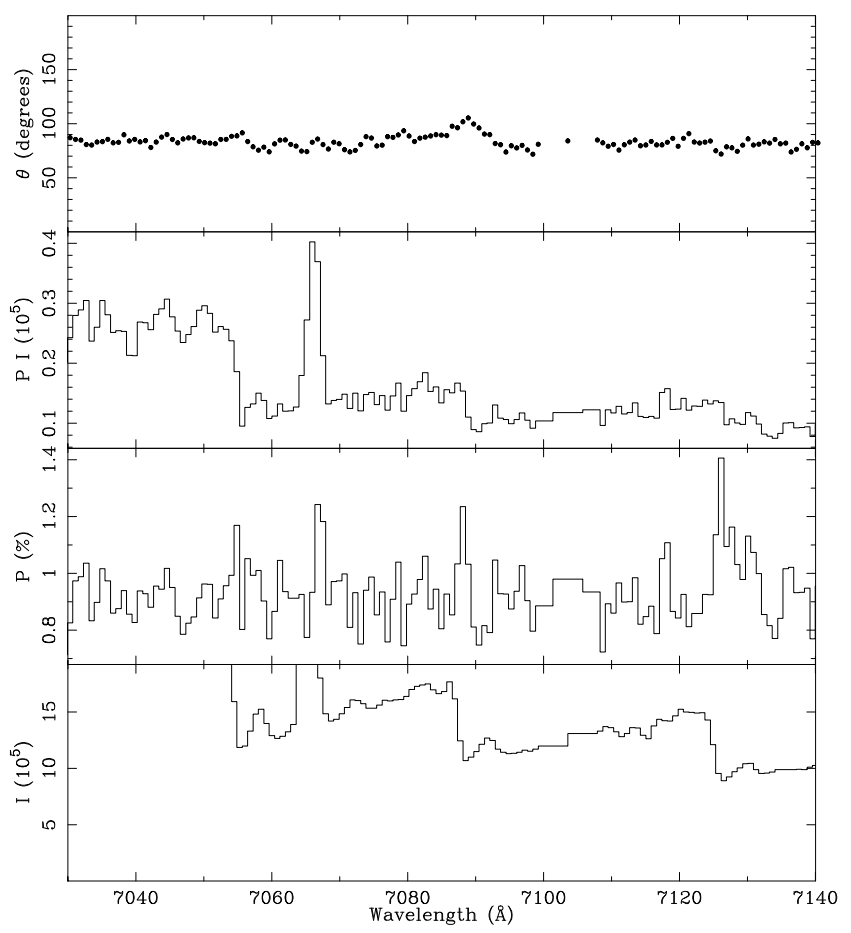

(iib) RX Pup 7082§

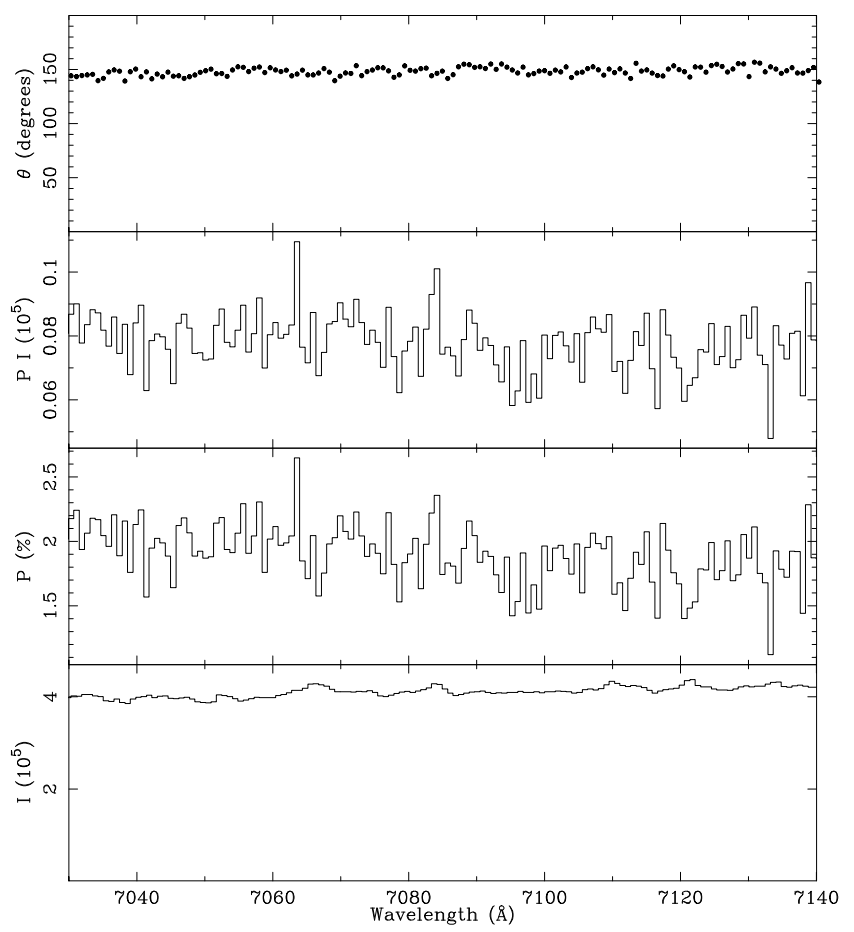

Fig. 9. Plots of the $\lambda 6825 \AA$ and $\lambda 7082 \AA$ observations of the survey stars. The polarization data are binned to a constant error of $0.5 \%$ in $\mathrm{P}$ (many spectra have smaller errors at their full resolution) 

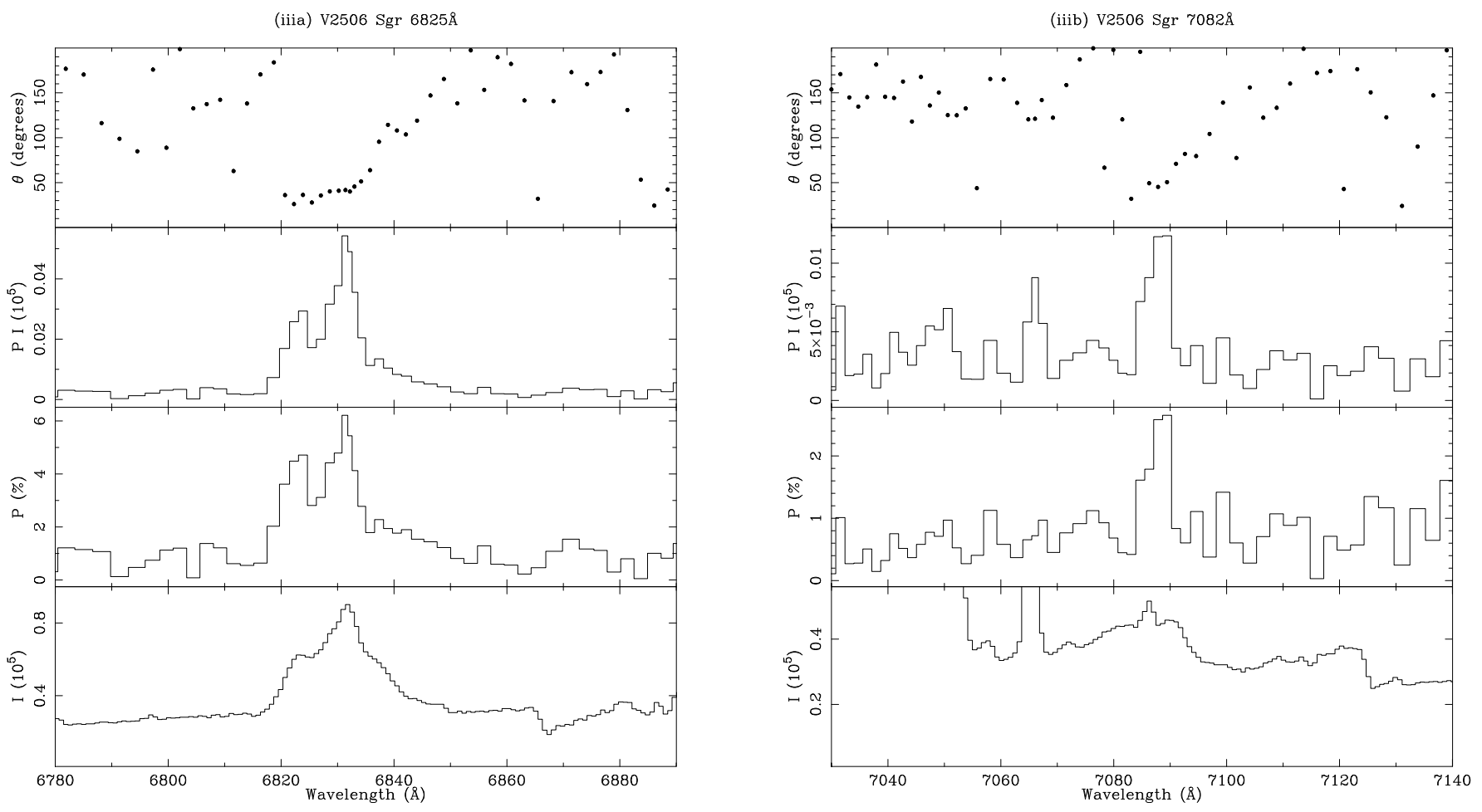

(iva) HK Sco $6825 \AA$

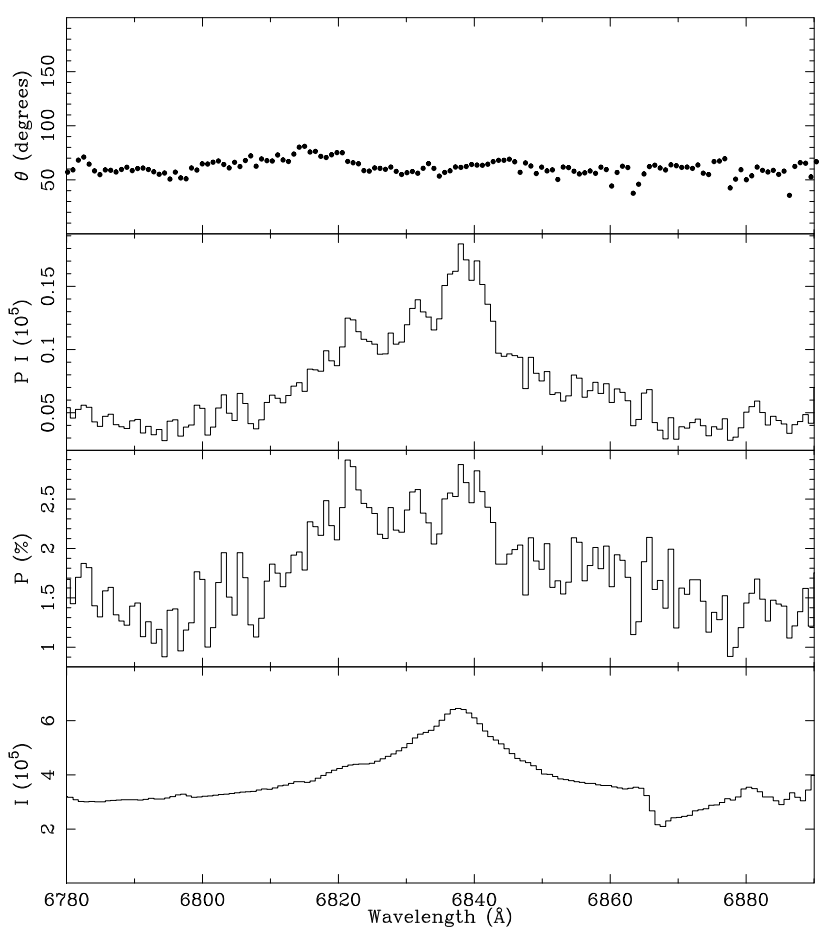

(ivb) HK Sco 7082尺

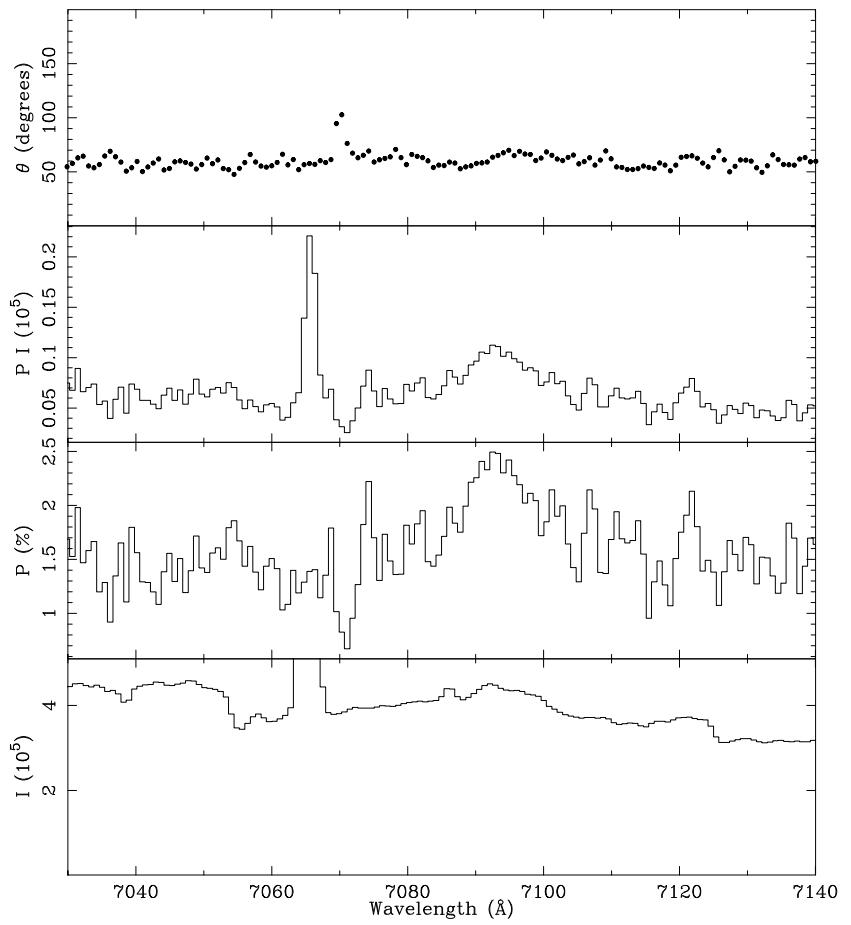

Fig. 9. continued 
(va) V455 Sco $6825 \AA$

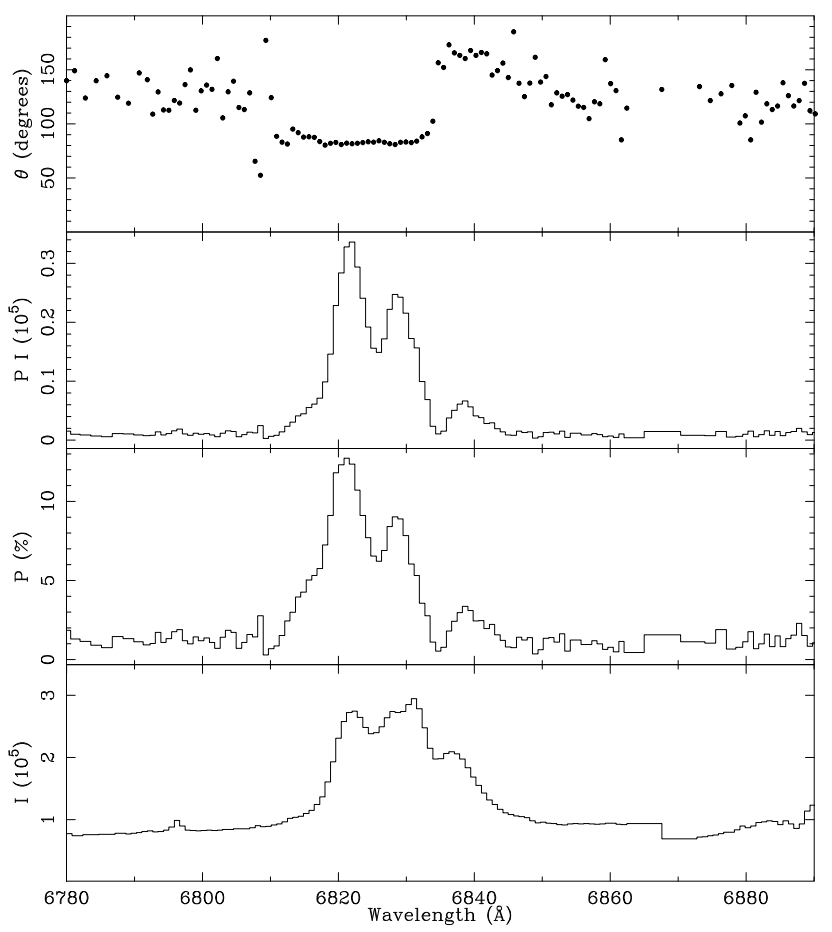

(via) RT Ser $6825 \AA$

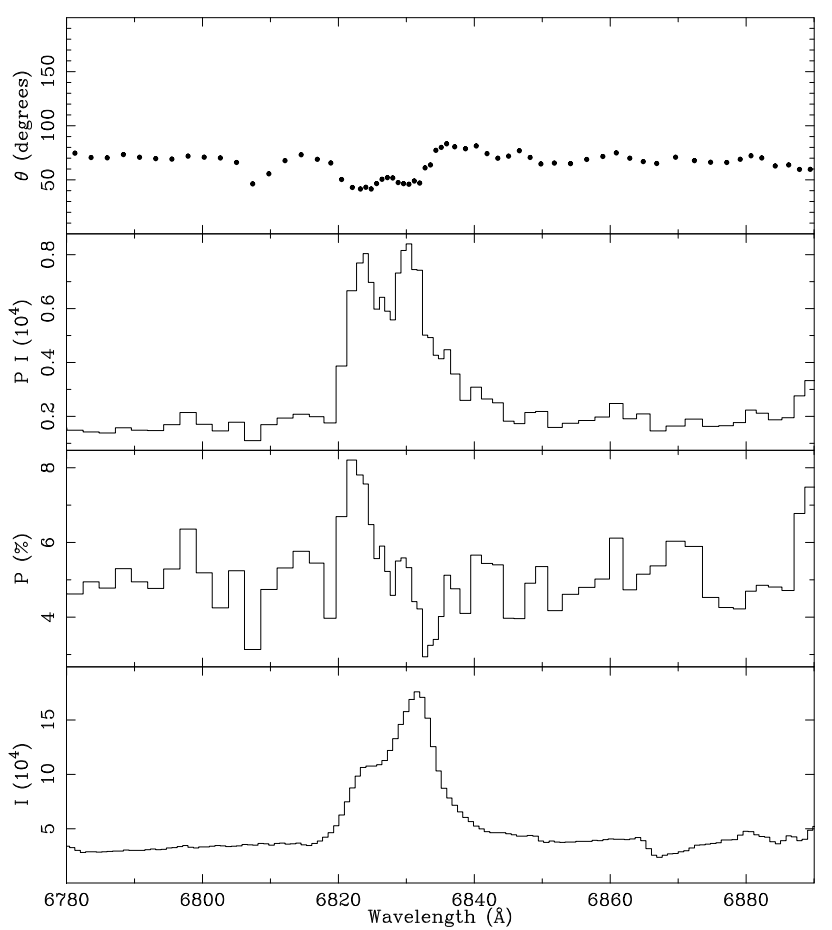

(vb) V455 Sco $7082 \AA$

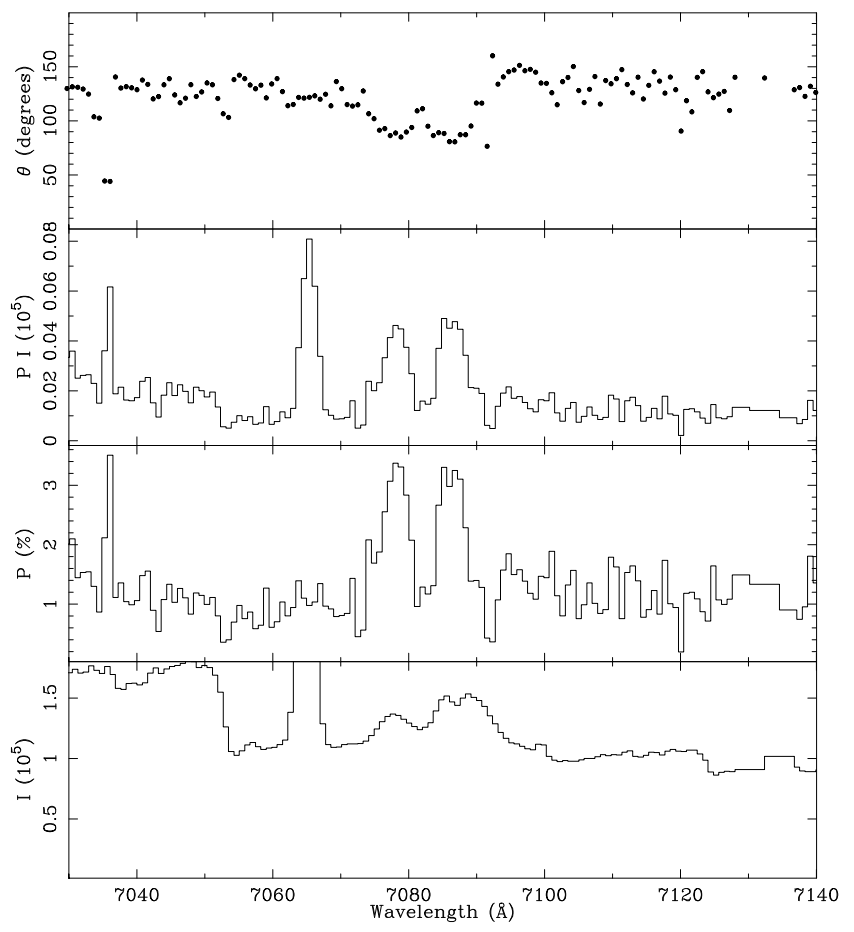

(vib) RT Ser 7082§

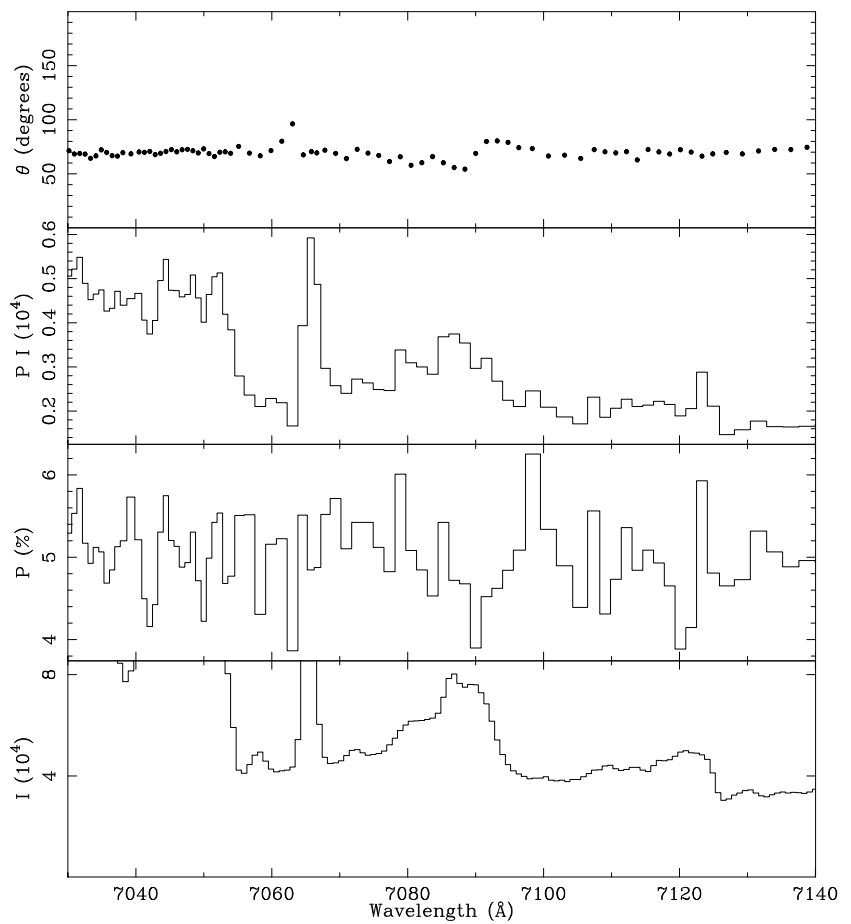

Fig. 9. continued 

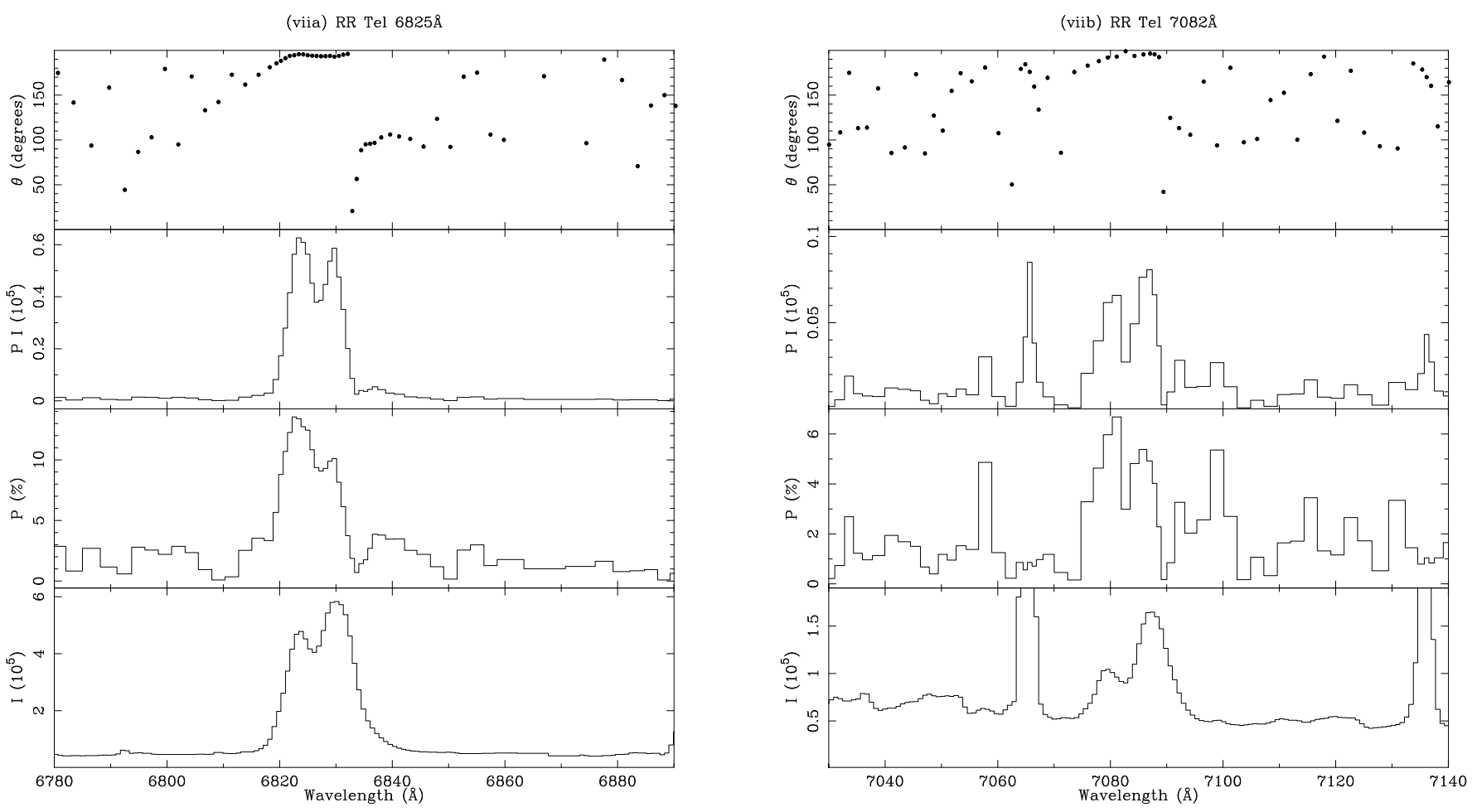

(viiia) CD-43 $143046825 \AA$

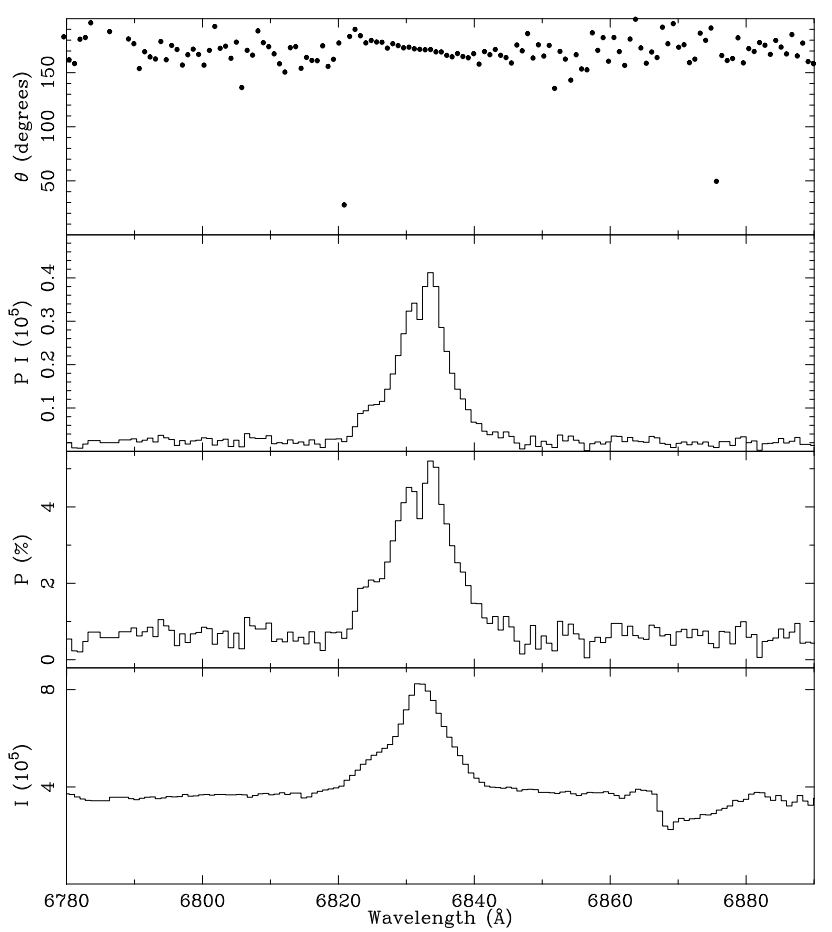

(viiib) $\mathrm{CD}-43143047082 \AA$

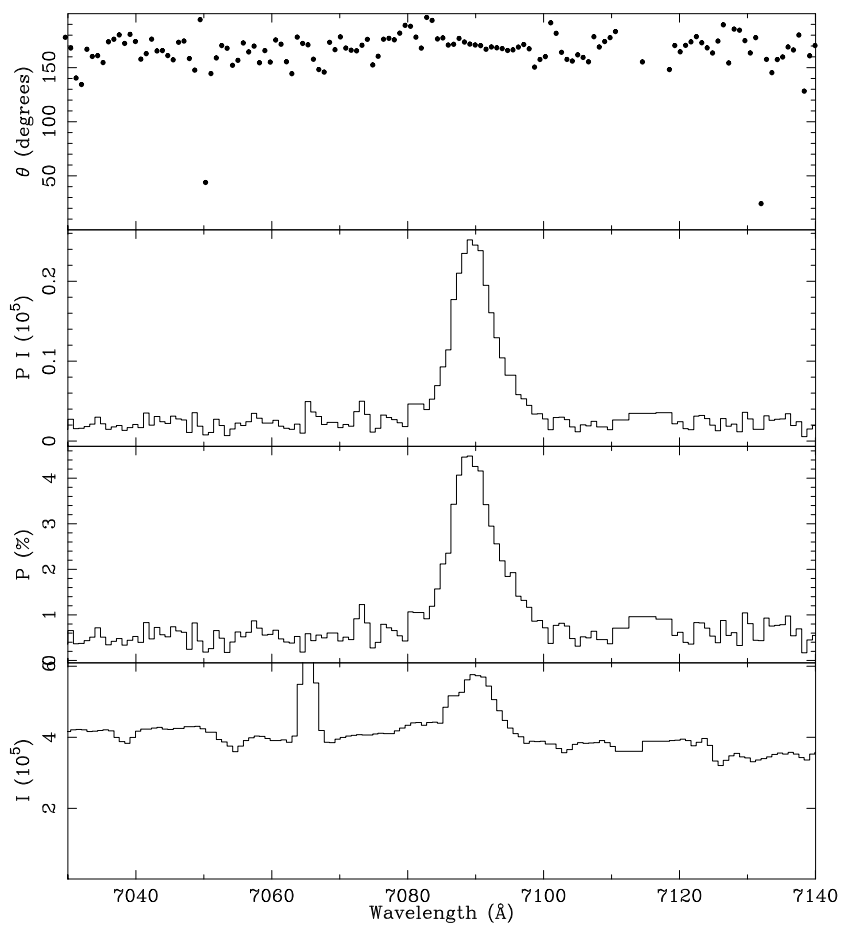

Fig. 9. continued 
(ixa) Ap3-1 6825

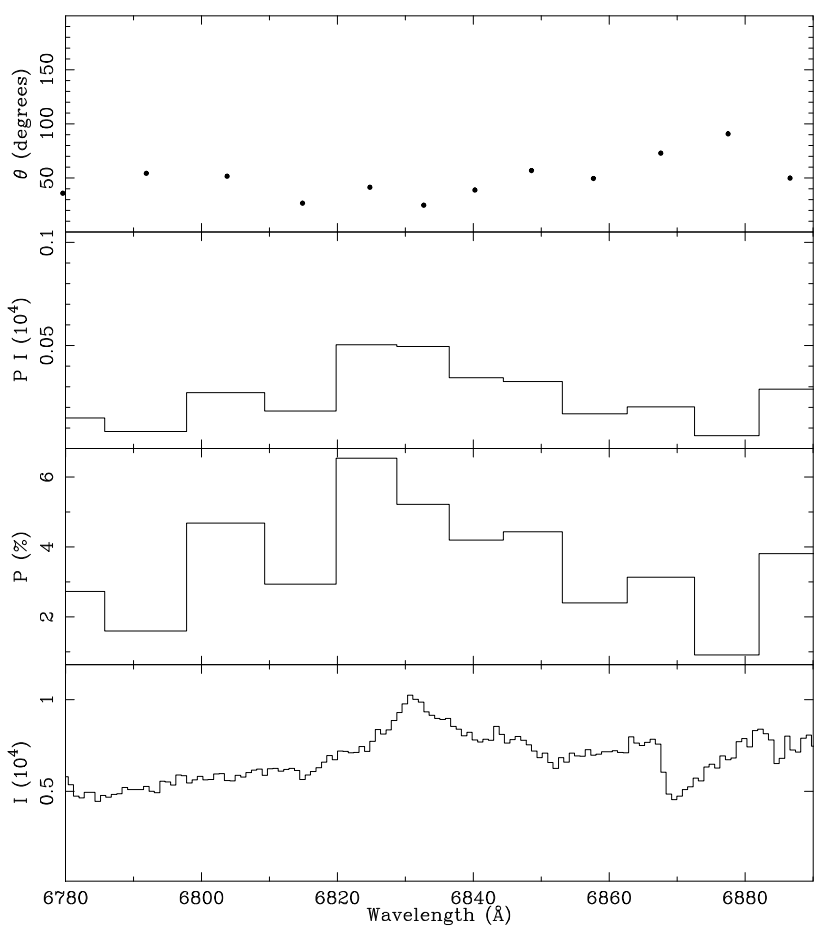

(xa) AS $2106825 \AA$

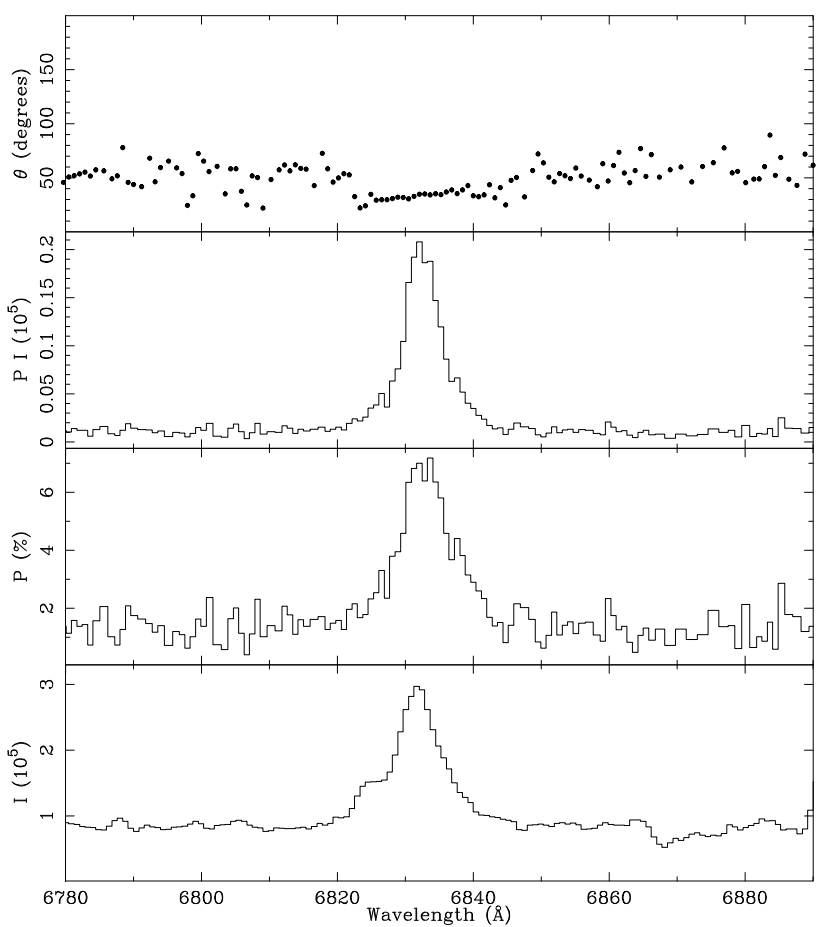

(ixb) Ap3-1 7082

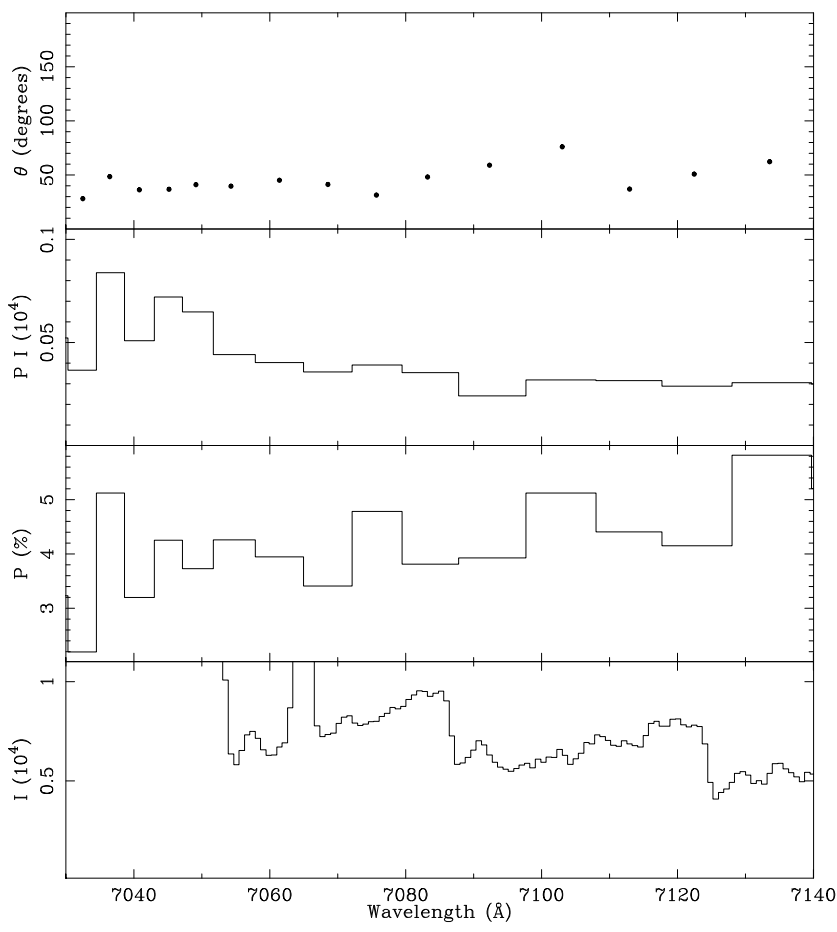

(xb) AS $2107082 \AA$

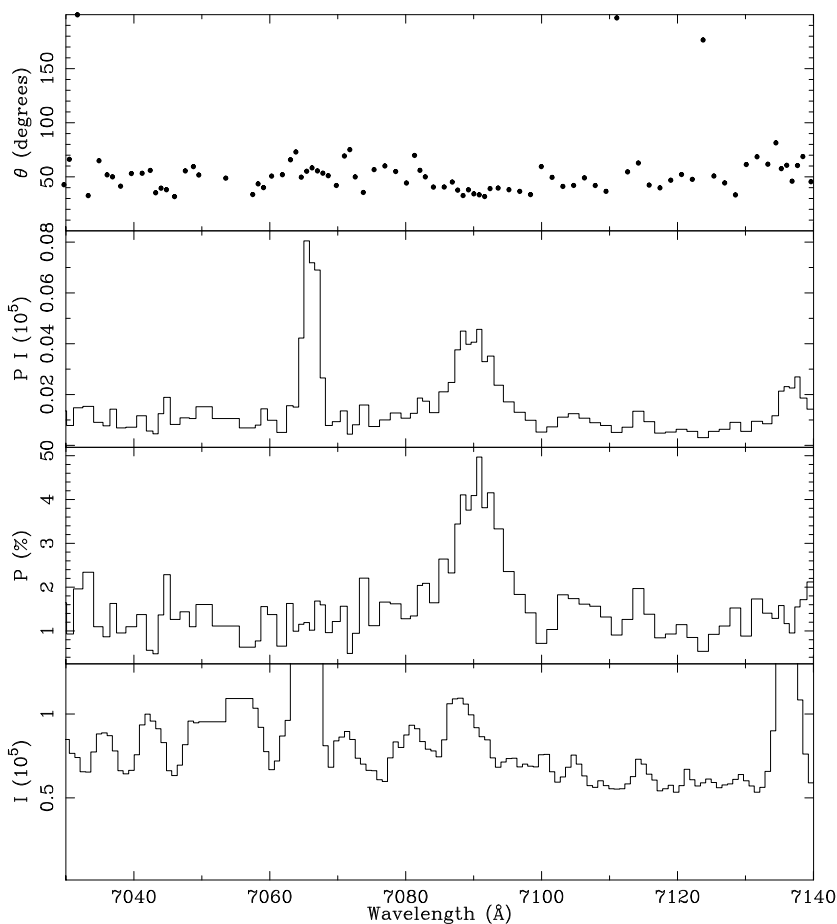

Fig. 9. continued 

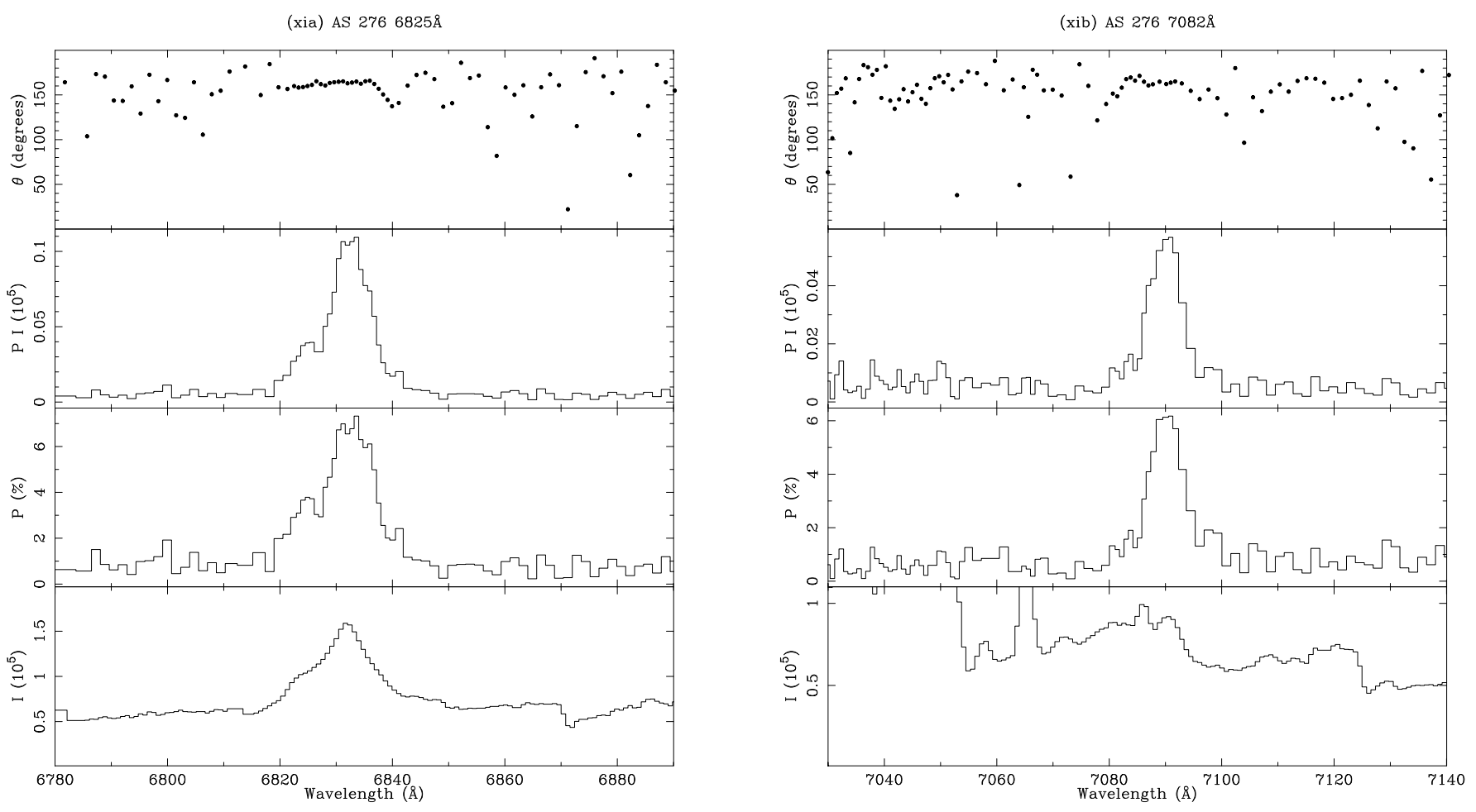

(xiia) AS $2816825 \AA$

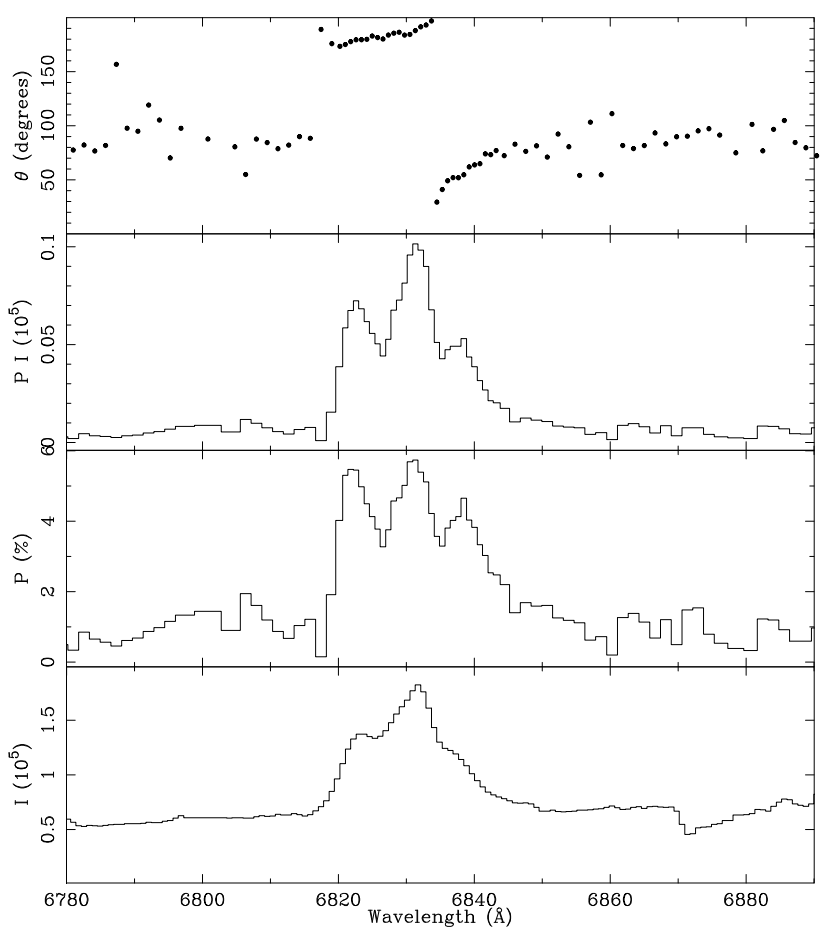

(xiib) AS $2817082 \AA$

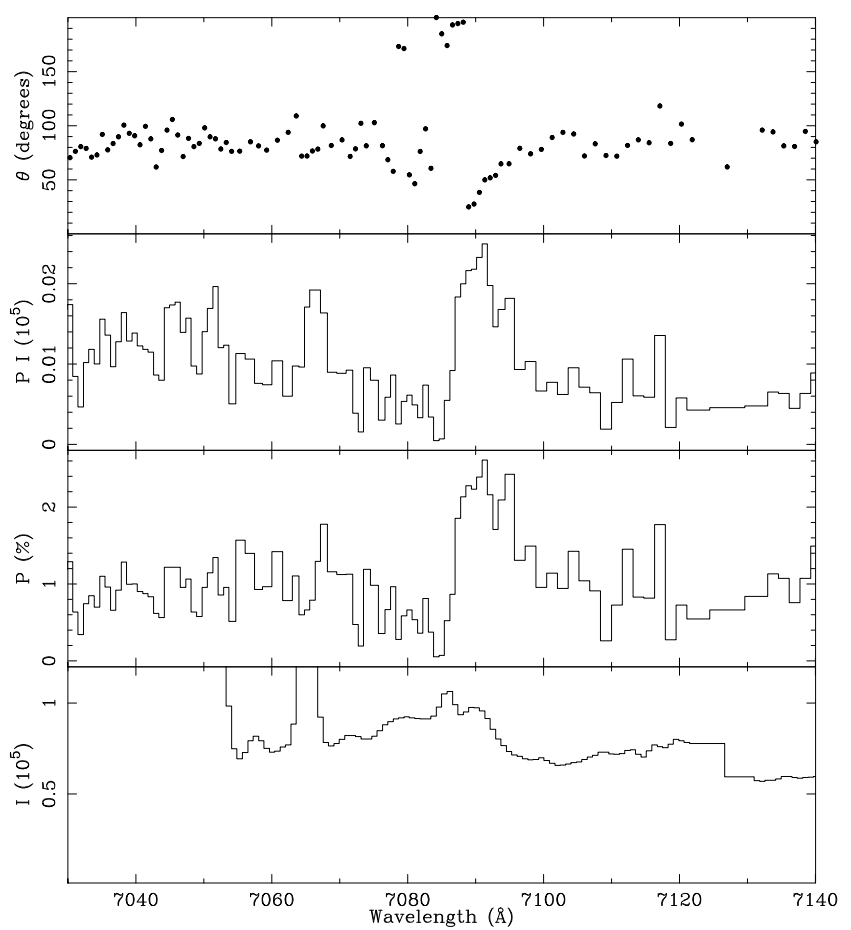

Fig. 9. continued 

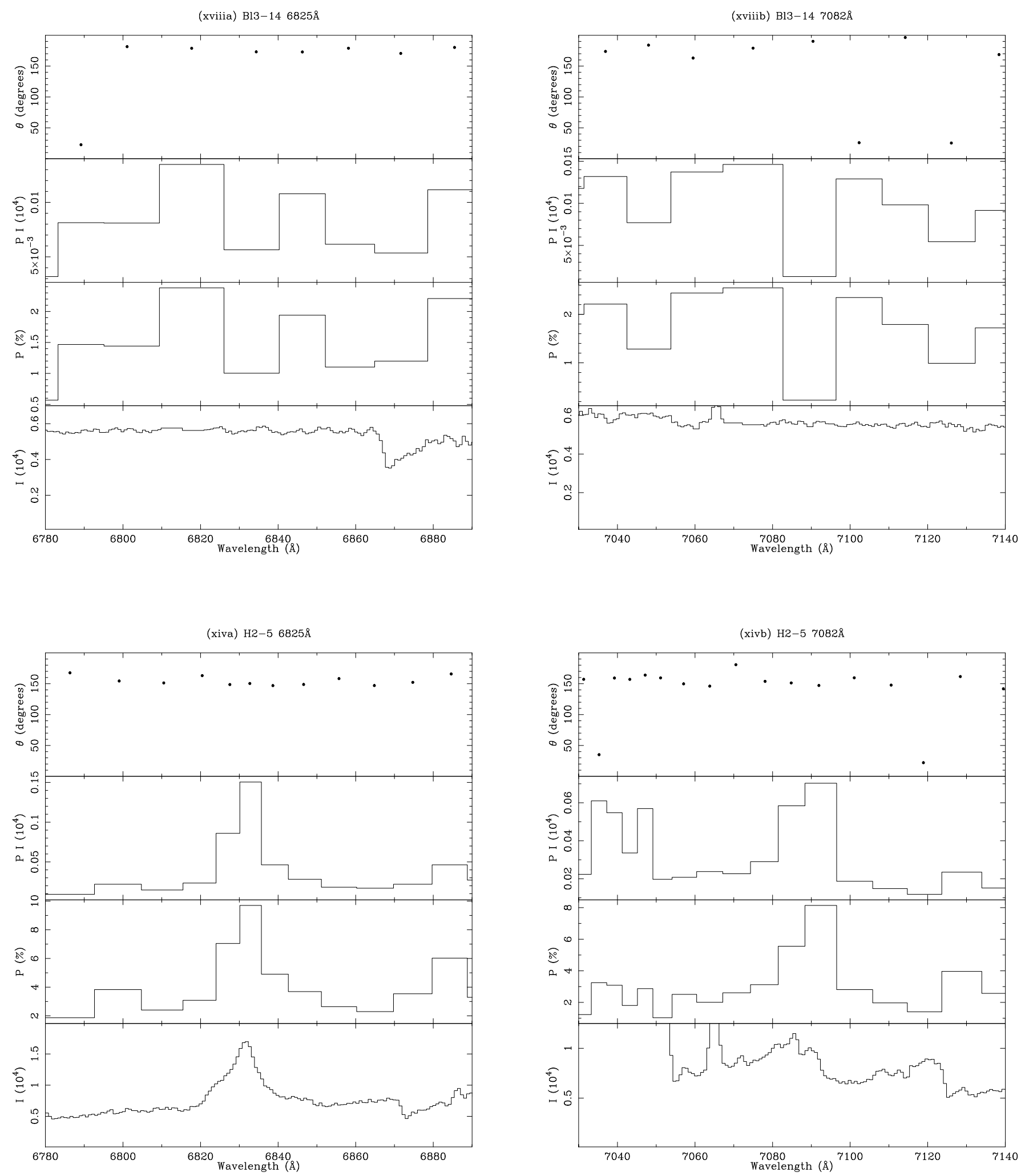

Fig. 9. concluded 

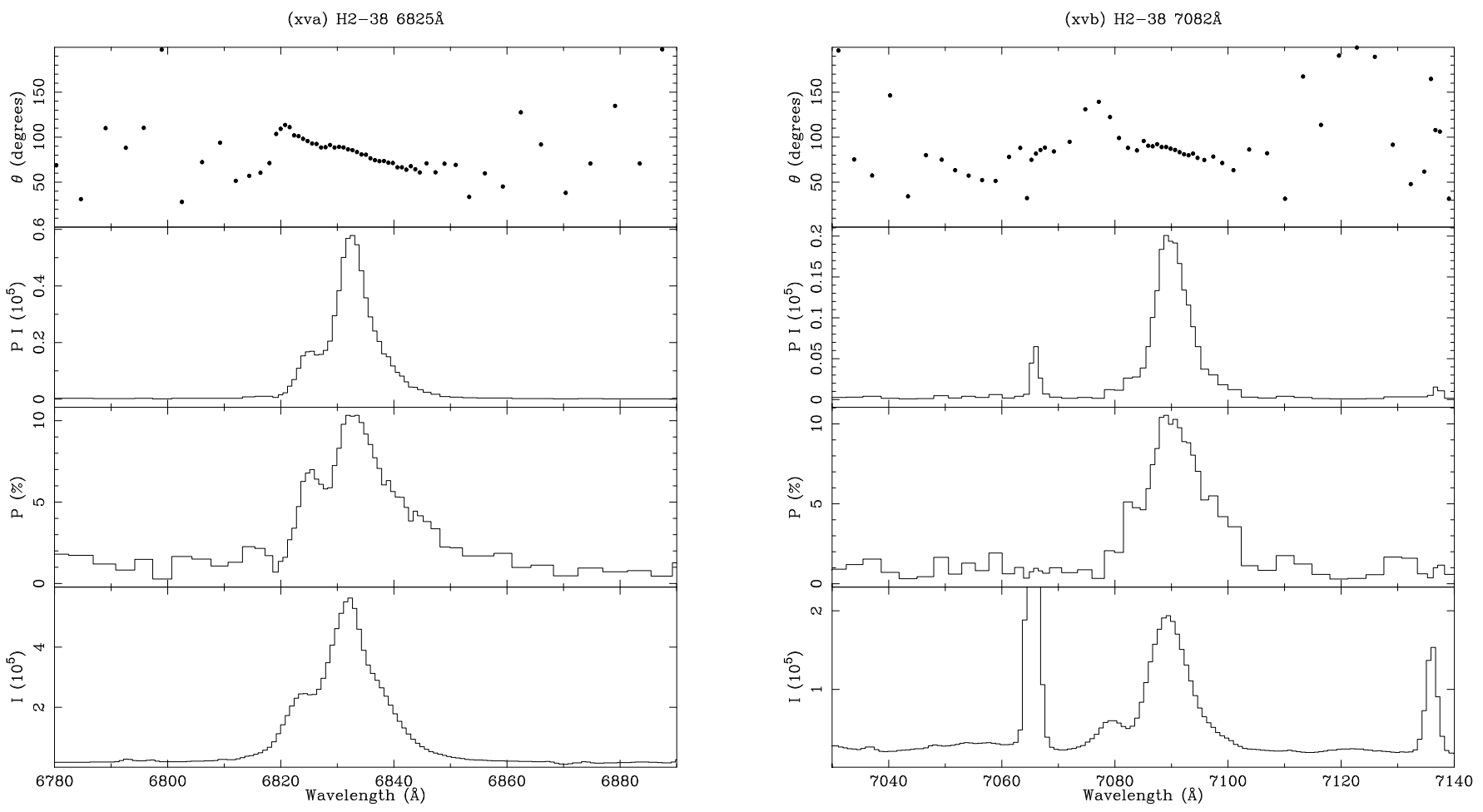

(xvia) He2-38 6825§

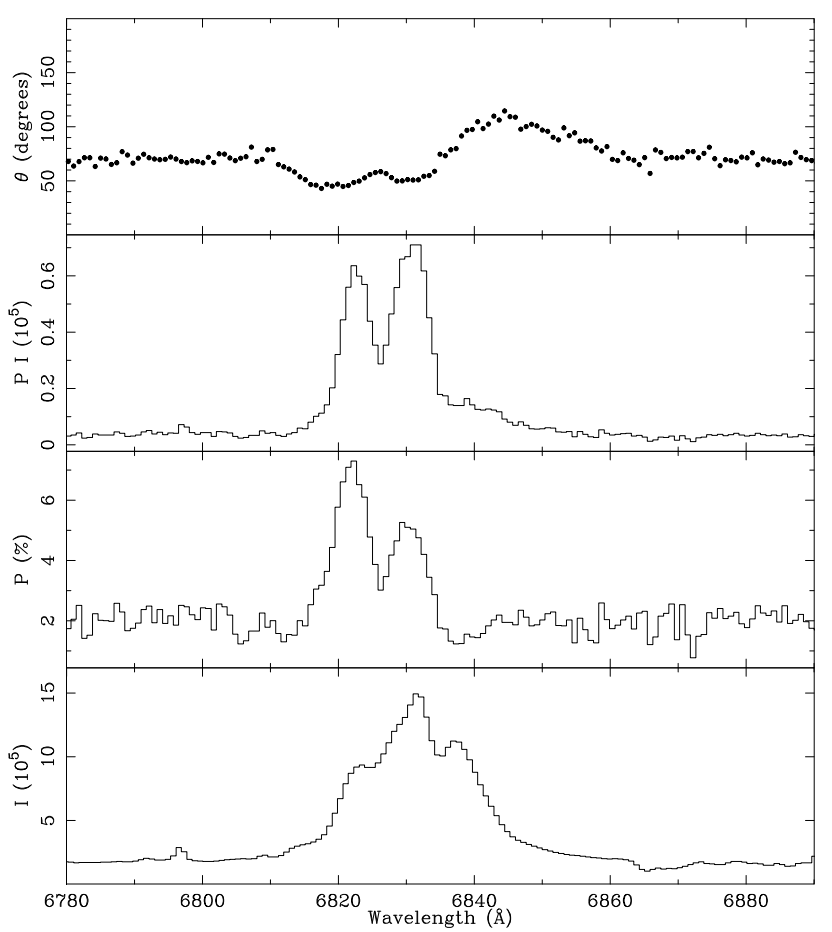

(xvib) $\mathrm{He} 2-38$ 7082尺

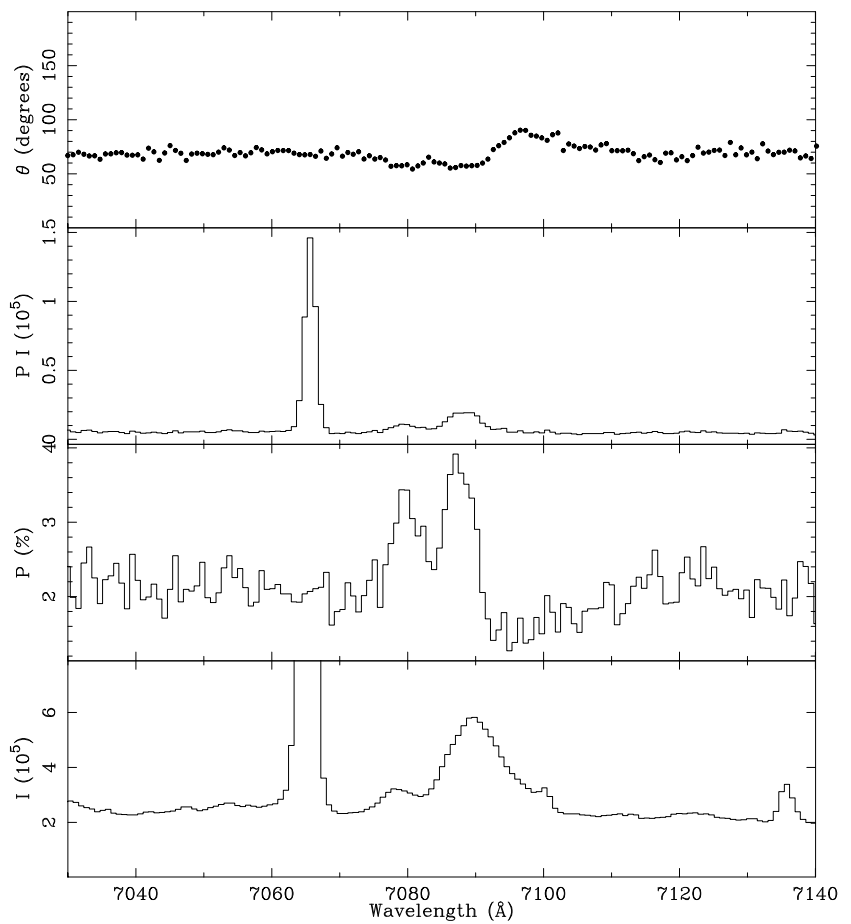

Fig. 9. concluded 
(xviia) He2-106 6825

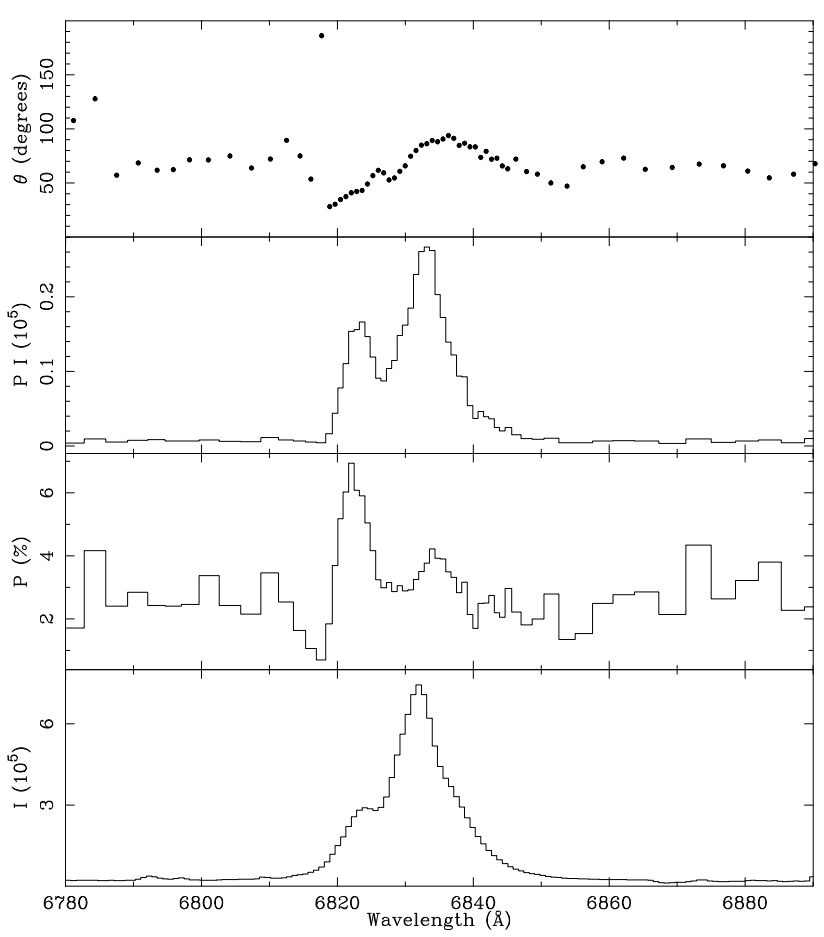

(xviiia) He2-127 6825

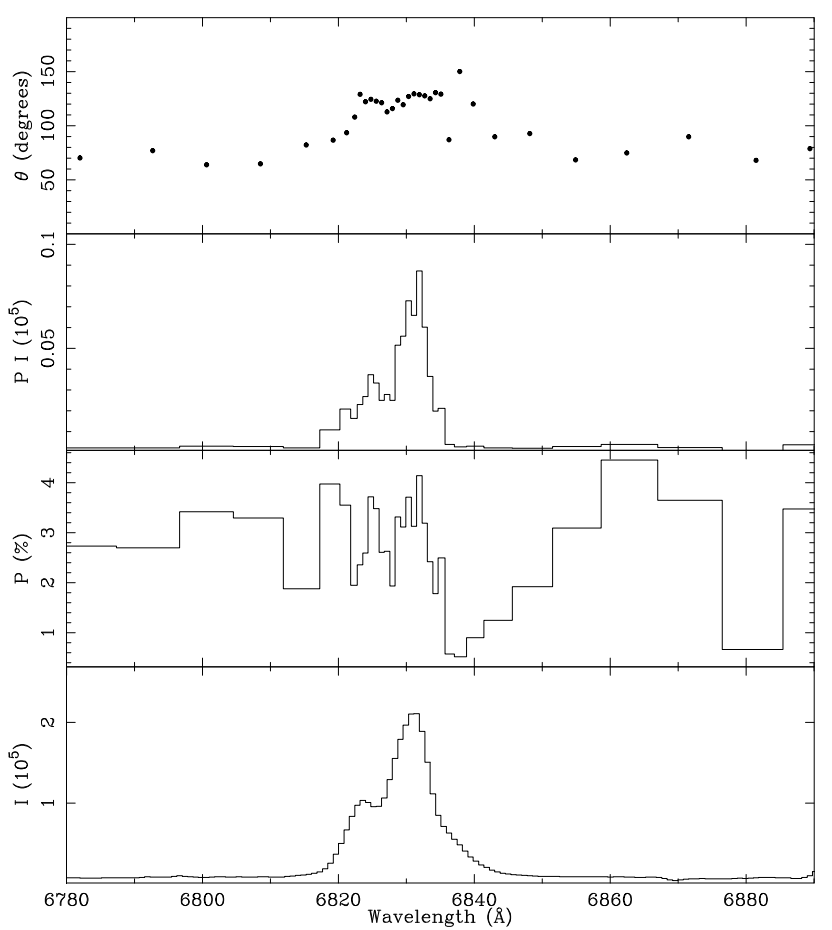

(xviib) $\mathrm{He} 2-1067082 \AA$

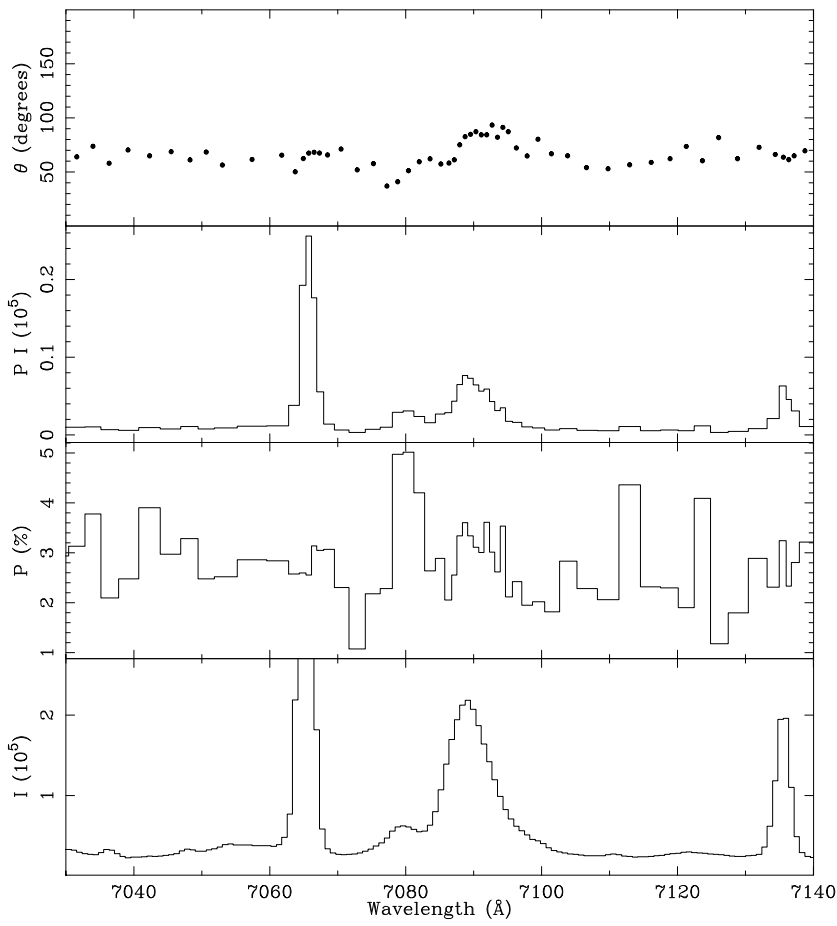

(xviiib) He2-127 7082A

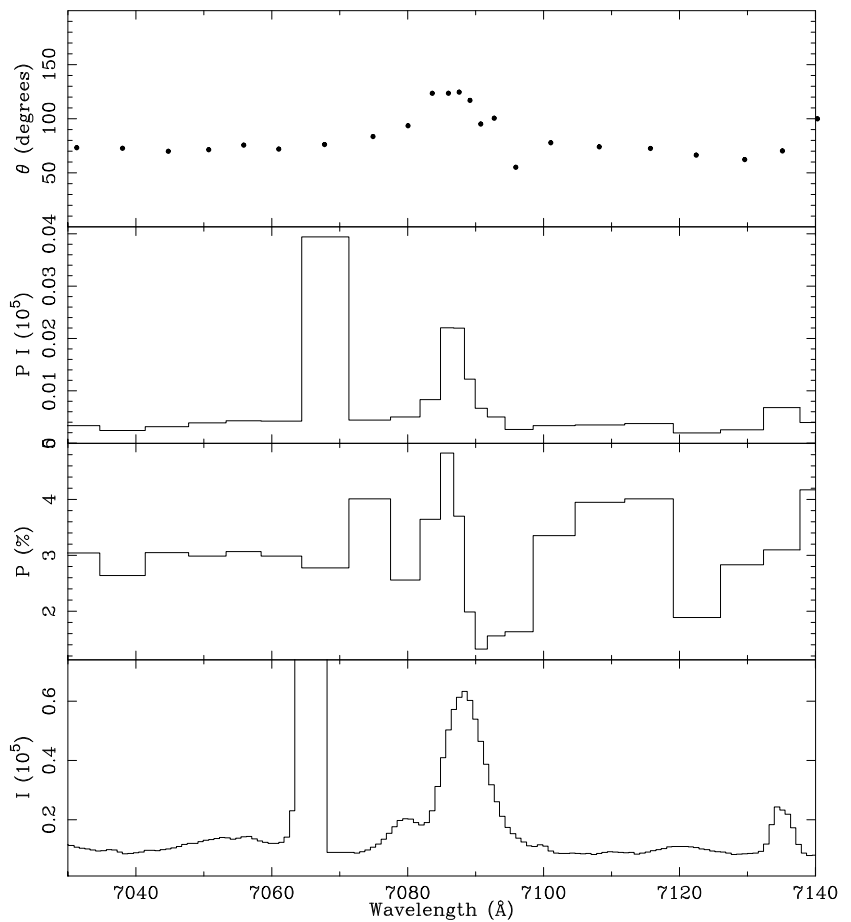

Fig. 9. concluded 

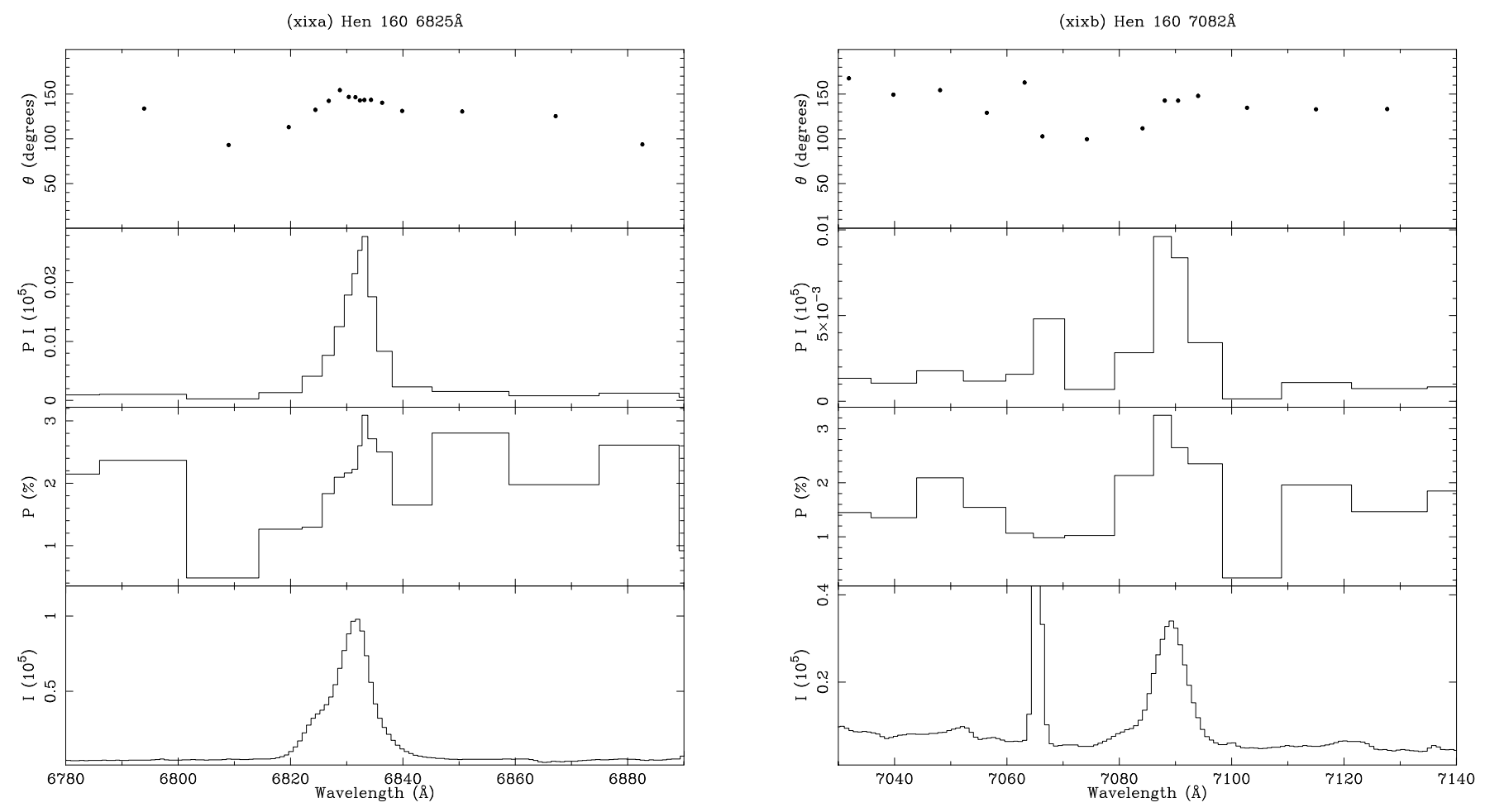

(xxa) Hen 828 6825
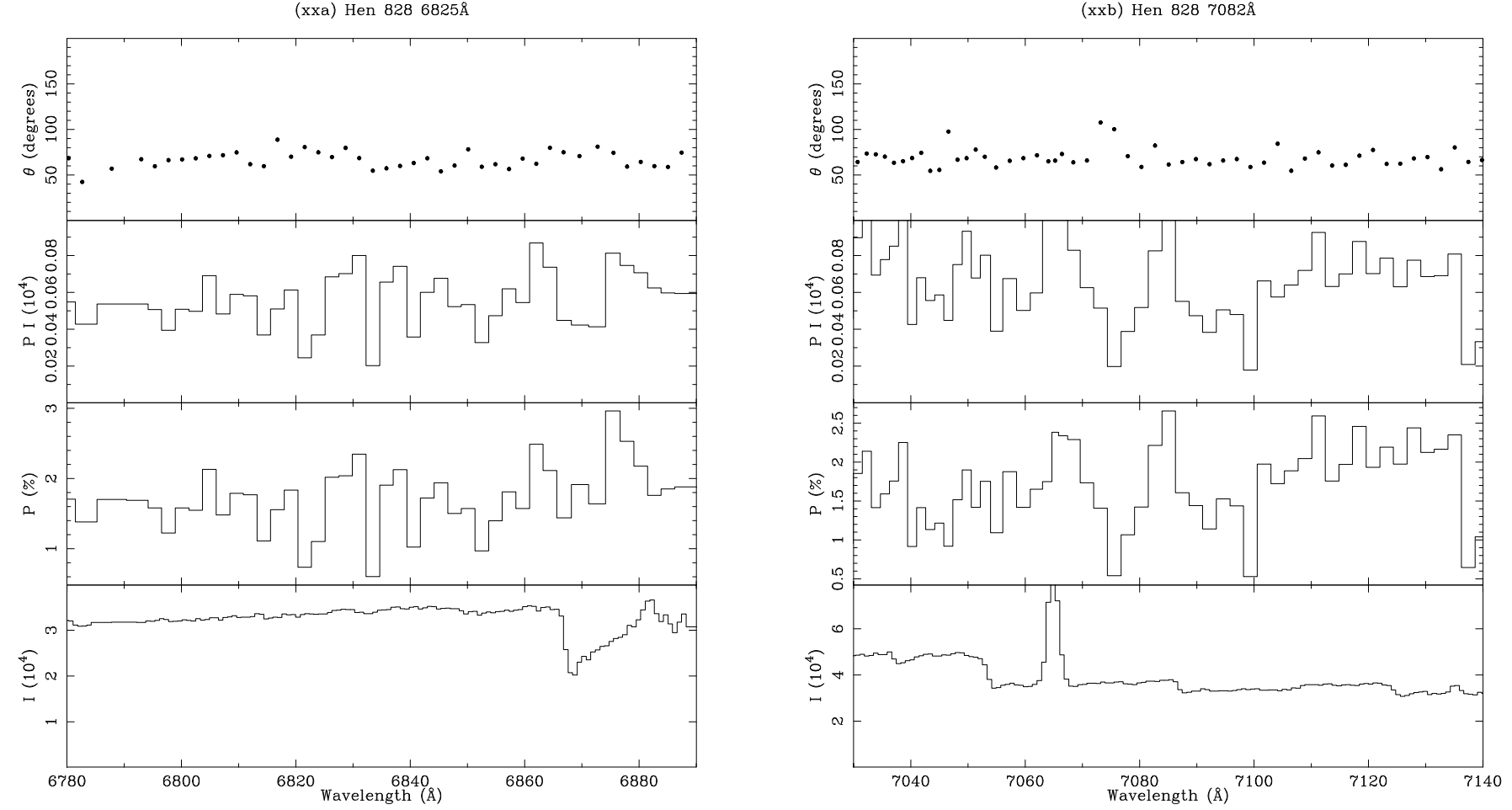

Fig. 9. concluded 
(xxia) Hen $9056825 \AA$

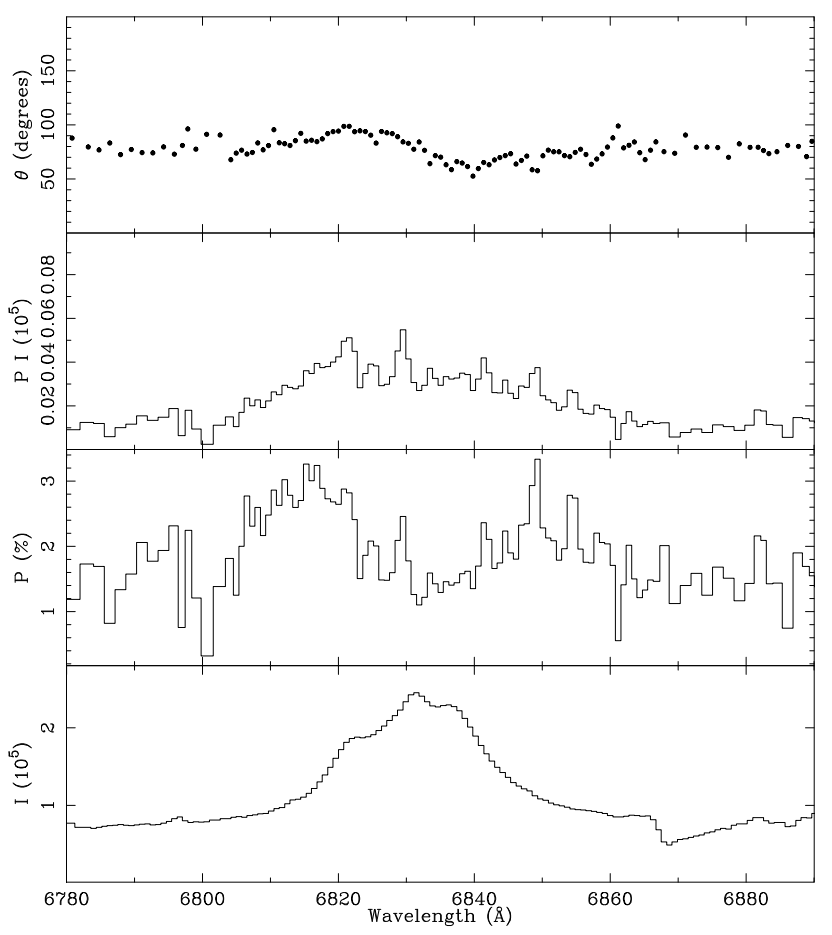

(xxiia) Hen 916 6825§

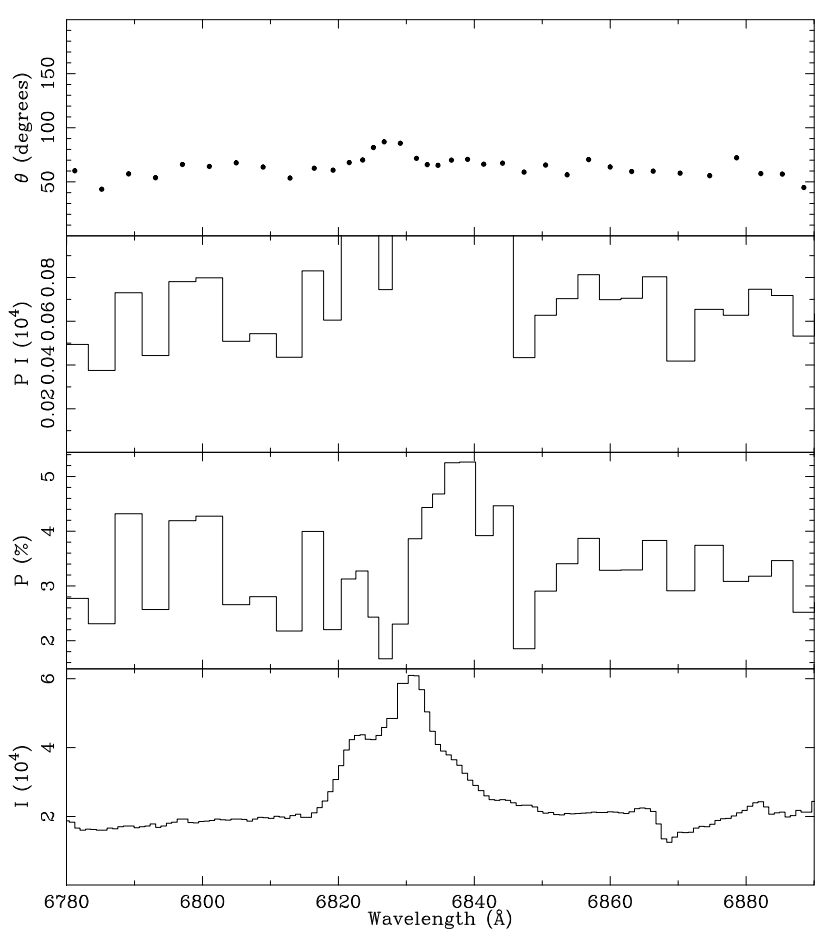

(xxib) Hen $9057082 \AA$

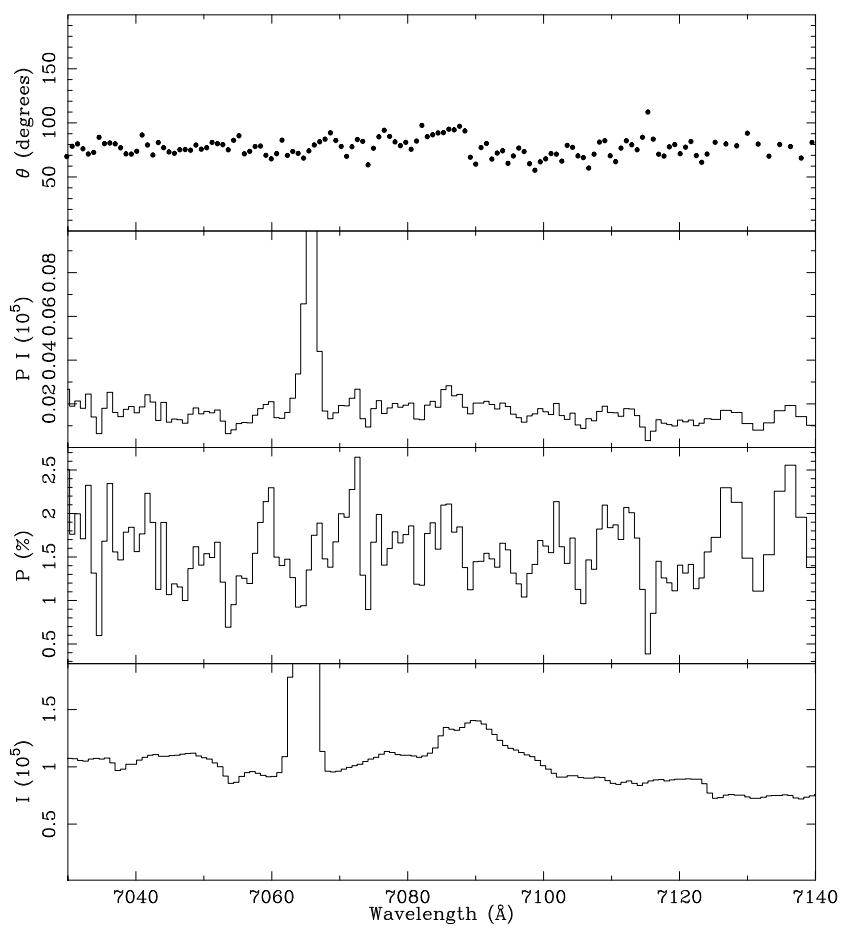

(xxiib) Hen $9167082 \AA$

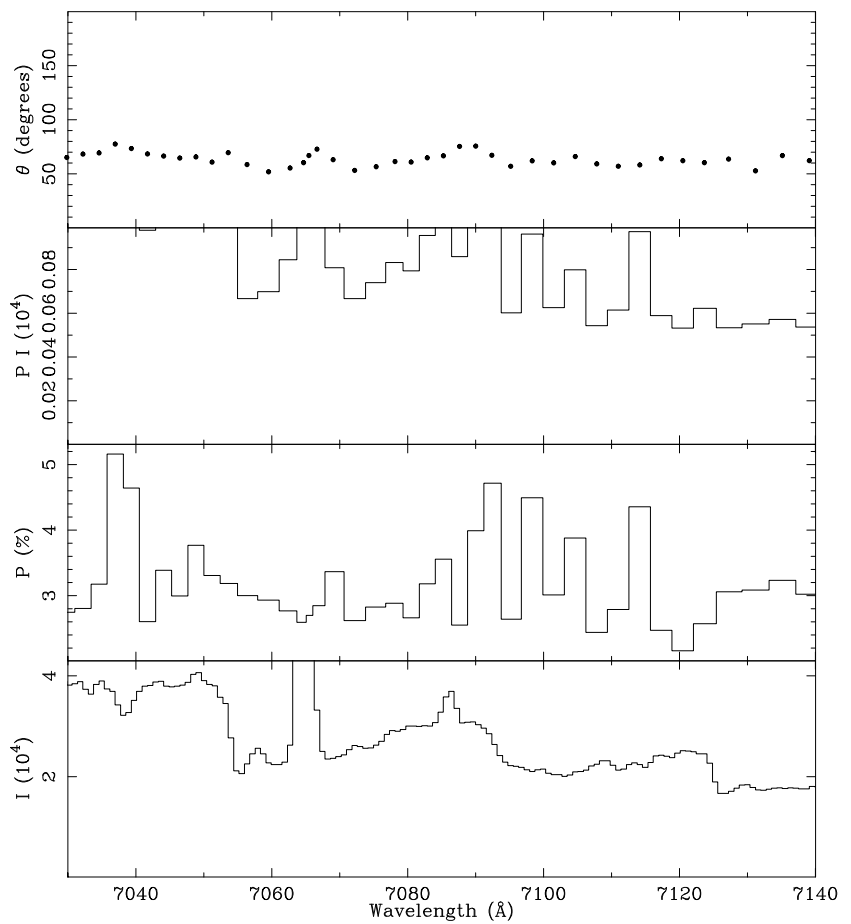

Fig. 9. concluded 

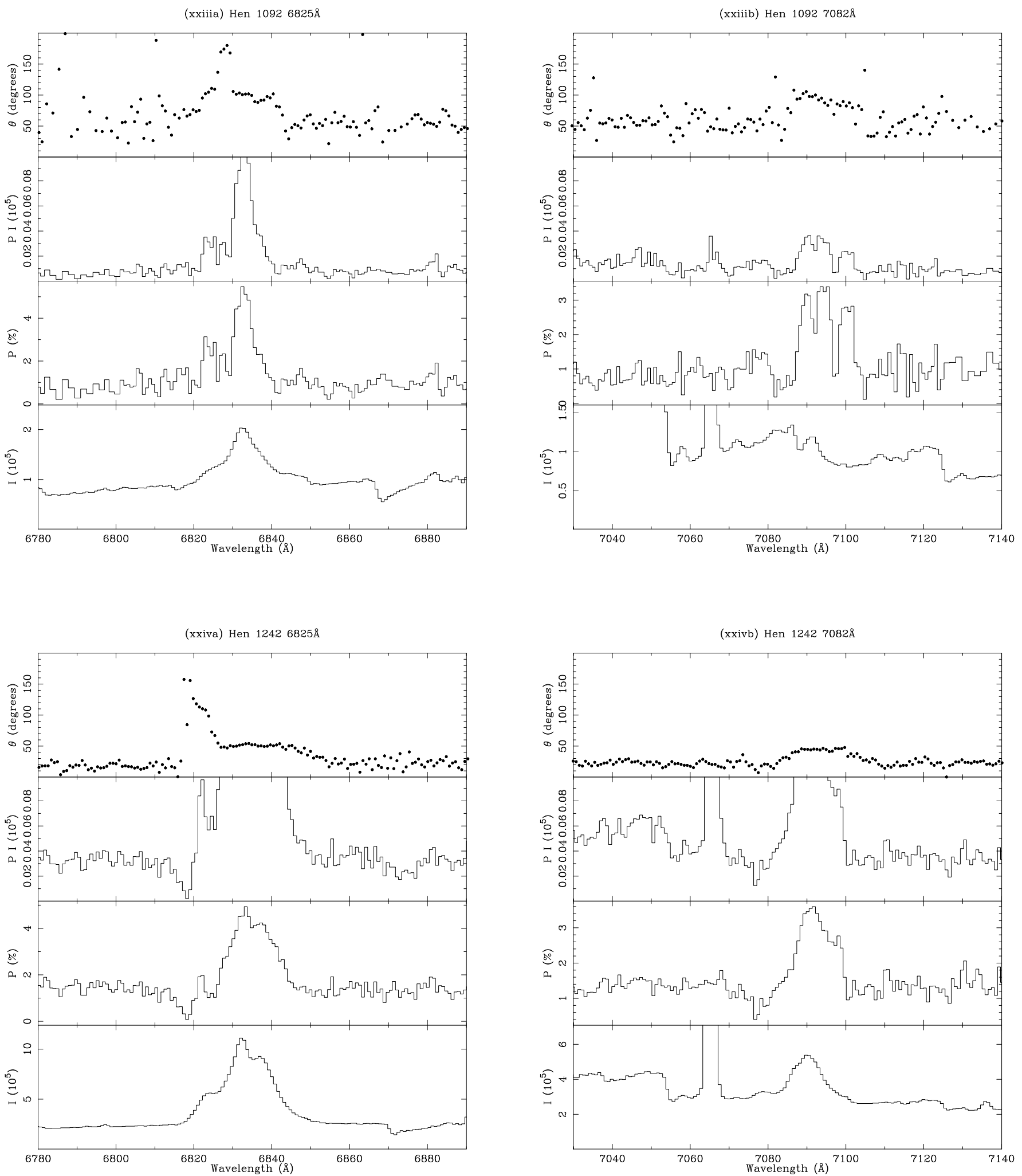

Fig. 9. concluded 
(xxva) Hen $13416825 \AA$

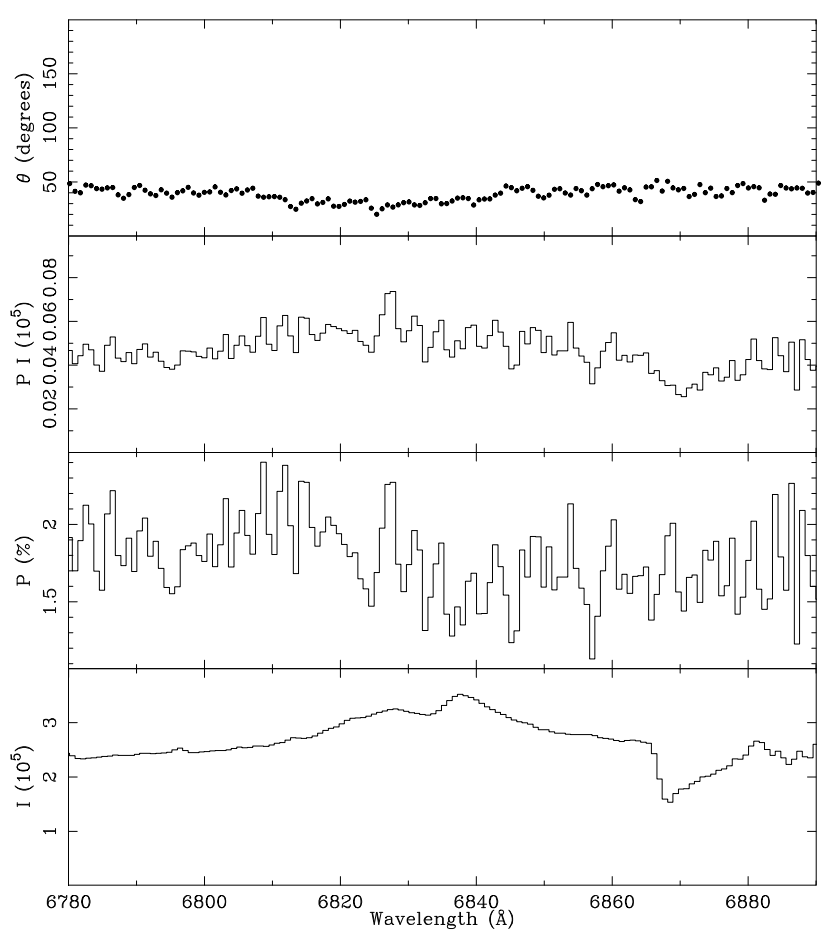

(xxvia) Hen $13426825 \AA$

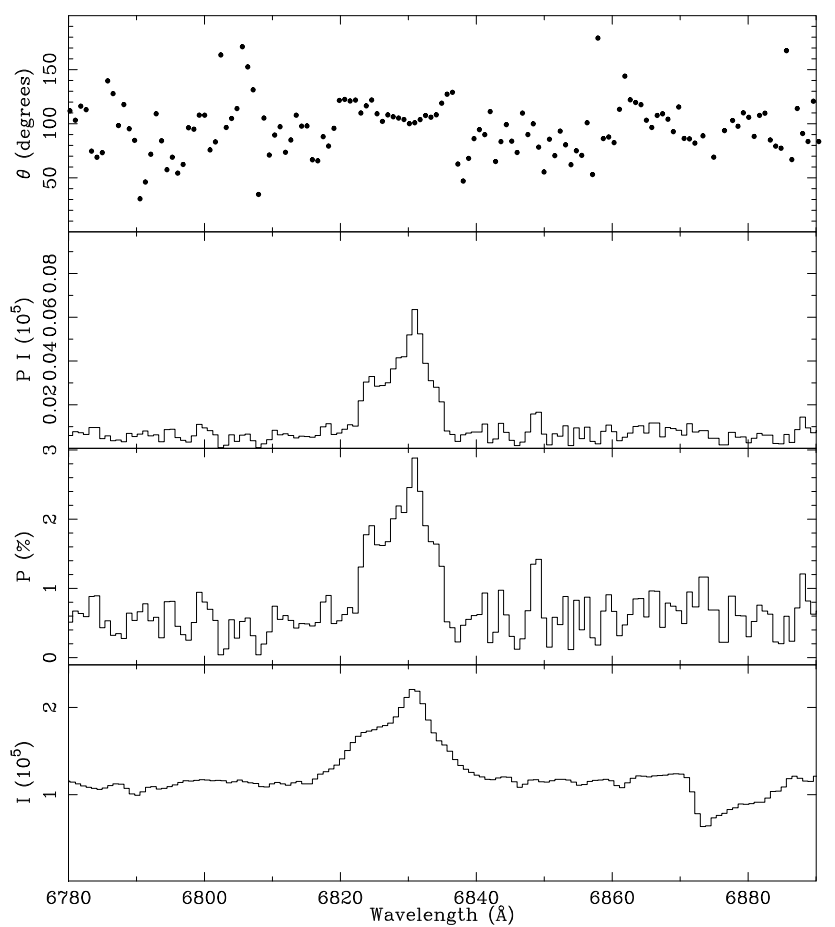

(xxvb) Hen 1341 7082A

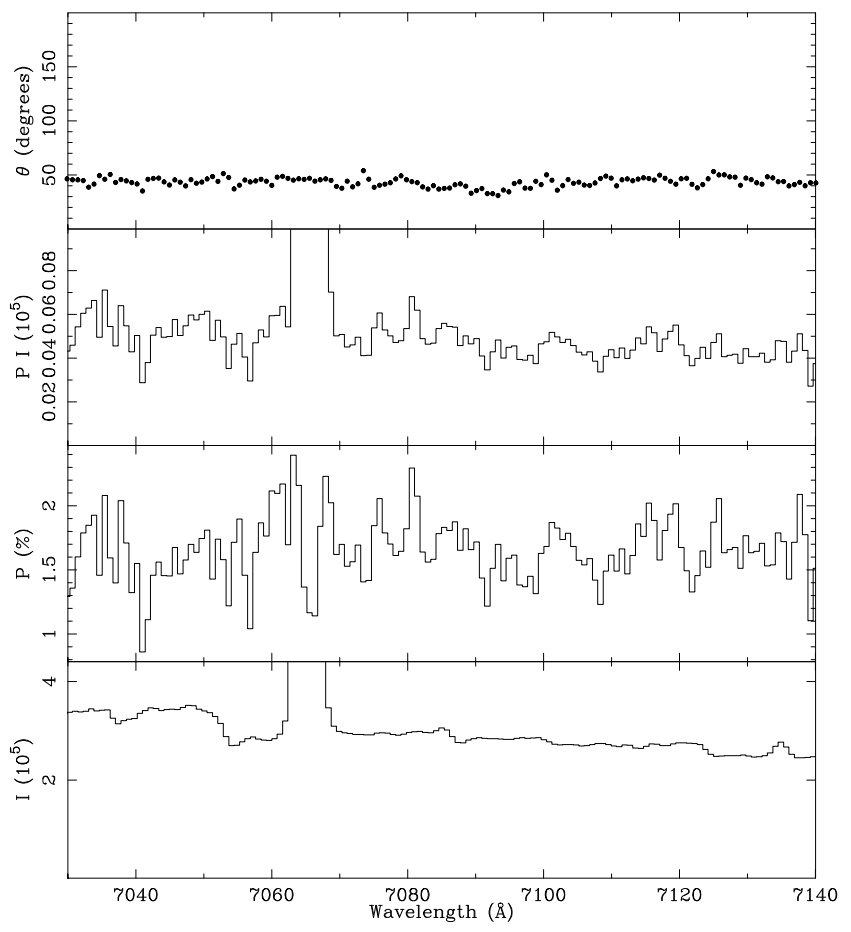

(xxvib) Hen $13427082 \AA$

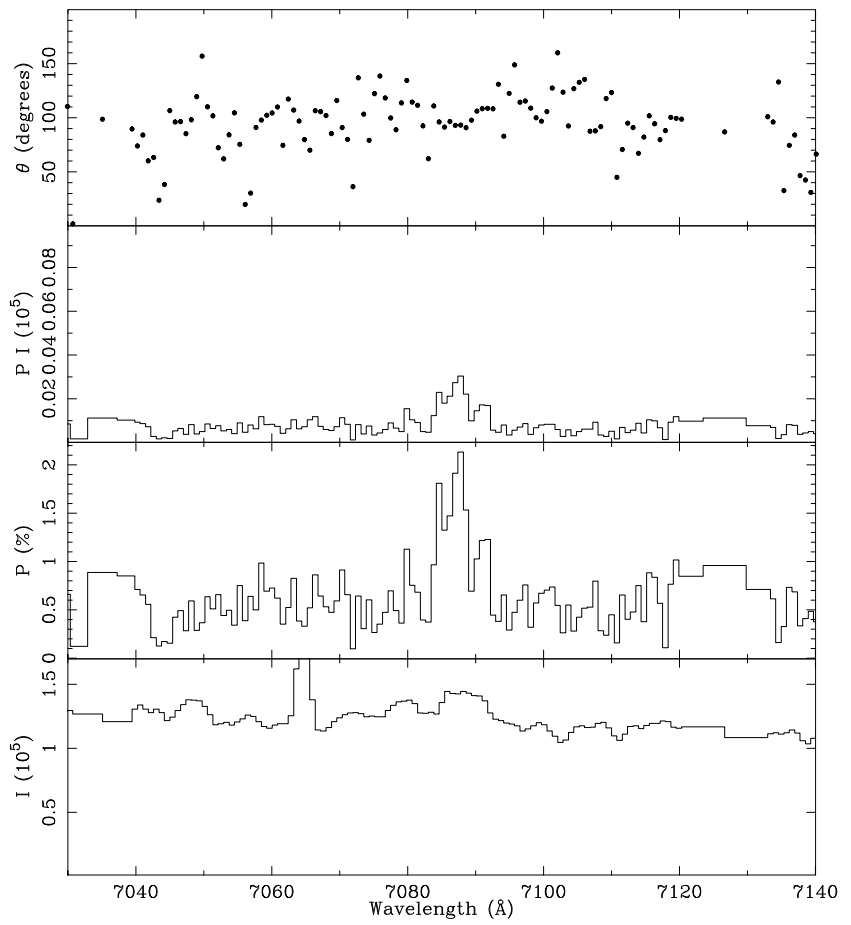

Fig. 9. concluded 

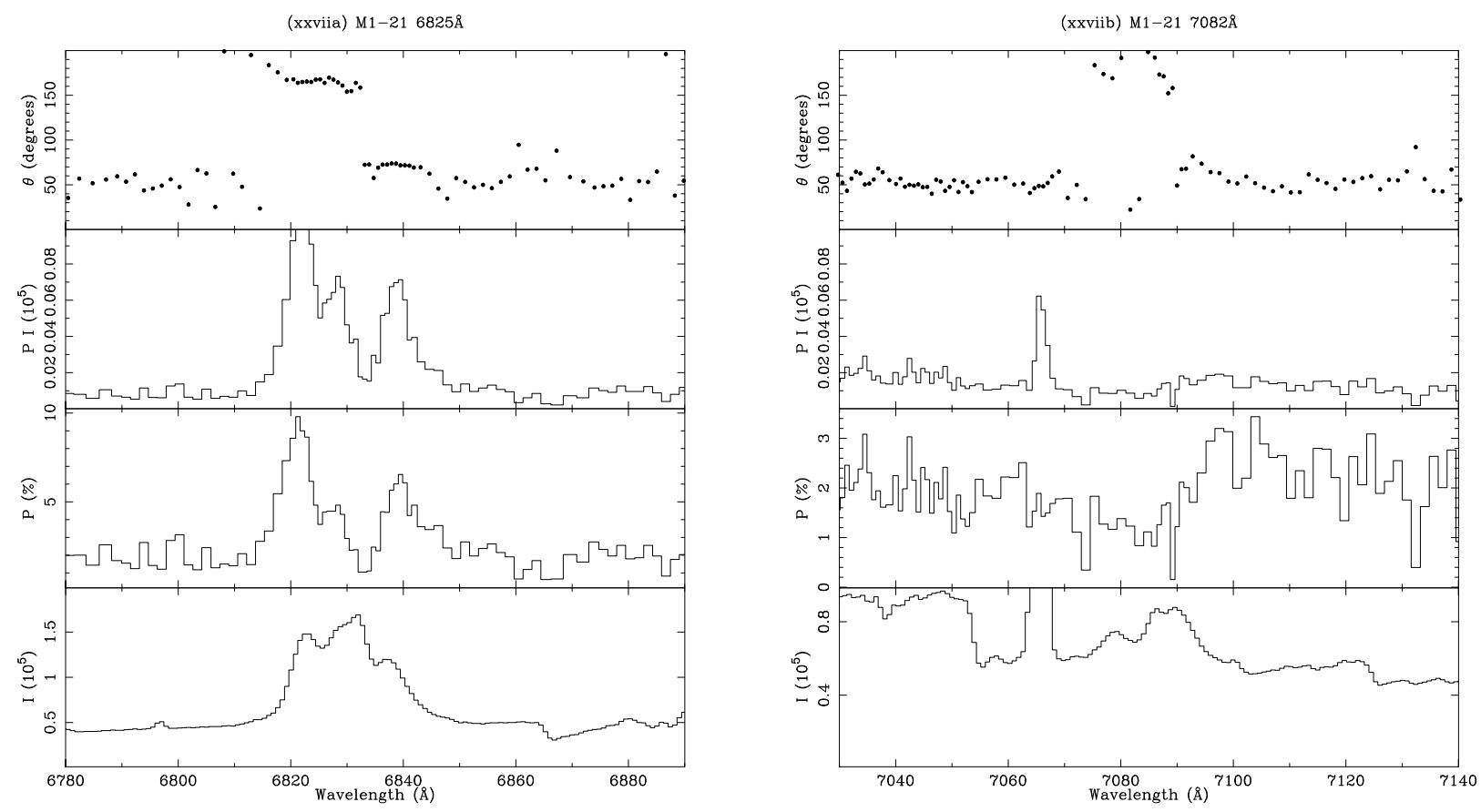

Fig. 9. concluded 